\title{
ON STRONGLY FLAT AND WEAKLY COTORSION MODULES
}

\author{
LEONID POSITSELSKI AND ALEXANDER SLÁVIK
}

\begin{abstract}
The aim of this paper is to describe the classes of strongly flat and weakly cotorsion modules with respect to a multiplicative subset or a finite collection of multiplicative subsets in a commutative ring. The strongly flat modules are characterized by a set of conditions, while the weakly cotorsion modules are produced by a generation procedure. Passing to the collection of all countable multiplicative subsets, we define quite flat and almost cotorsion modules, and show that, over a Noetherian ring with countable spectrum, all flat modules are quite flat and all almost cotorsion modules are cotorsion.
\end{abstract}

\section{Contents}

1. Introduction 1

2. Obtainable Modules 10

3. $S$-Contramodule $R$-Modules 14

4. Rings with a Multiplicative Subset 20

5. Rings with Several Multiplicative Subsets 23

6. Finite-Dimensional Noetherian Rings with Countable Spectrum 29

7. Contramodule Approximation Sequences 35

8. Quite Flat and Almost Cotorsion Modules 44

References $\quad 50$

\section{INTRODUCTION}

The aim of the theory developed in this paper is to describe flat modules over associative rings. Another related goal is to describe Enochs cotorsion modules (see the discussion below in Section 1.1). These goals, obviously important, are difficult to achieve in a substantial enough yet fully general way. In this paper we provide such descriptions for Noetherian commutative rings with countable spectrum (see Sections 1.5-1.6). We also describe certain subclasses of flat modules and overclasses of Enochs cotorsion modules over commutative rings.

It needs to be explained what is meant by "describing flat modules". One such description is provided by the Govorov-Lazard theorem [14, 18] claiming that, for any associative ring $R$, the flat left $R$-modules are precisely the filtered inductive limits of finitely generated projective (or free) $R$-modules. The following example illustrates the difference between the Govorov-Lazard theorem and our approach. 
Let $R=\mathbb{Z}$ be the ring of integers. Then the flat $R$-modules are simply the torsionfree abelian groups. The Govorov-Lazard theorem tells that these are precisely the filtered inductive limits of finitely generated free abelian groups. This assertion is obvious, though: any torsion-free abelian group is the directed union of its finitely generated subgroups, which are free abelian groups. Classifying torsion-free abelian groups is a hopeless task; still, one can describe them in a way much more nontrivial than the one provided by the Govorov-Lazard theorem.

The description that we have in mind claims, in the case of the ring $R=\mathbb{Z}$, that any torsion-free abelian group $F$ is a direct summand of an abelian group $G$ for which there exists a short exact sequence $0 \longrightarrow U \longrightarrow G \longrightarrow V \longrightarrow 0$, where $U$ is a free abelian group and $V$ is a $\mathbb{Q}$-vector space. It is this result, going back to Harrison [17, Section 2], Matlis [20, Theorem 4.1], and Trlifaj [31, Proposition 2.8], that we seek to extend to more complicated commutative rings $R$ in this paper.

In a way of further motivation, let us say that flat modules over commutative rings are obviously of interest in algebraic geometry; and Enochs cotorsion modules over commutative rings have a geometric significance, too, as the modules of cosections of locally cotorsion contraherent cosheaves over affine schemes [22] (cf. the discussion in the introduction to [23]).

1.1. Before proceeding to formulate the main results of this paper, let us give a more detailed historical overview.

The word "cotorsion" was introduced to homological algebra by Harrison [17], who called "co-torsion abelian groups" the abelian groups $C$ such that $\operatorname{Hom}_{\mathbb{Z}}(\mathbb{Q}, C)=0=$ $\operatorname{Ext}_{\mathbb{Z}}^{1}(\mathbb{Q}, C)$. Subsequently Matlis $[20]$ studied, under the name of cotorsion modules over a commutative integral domain $R$, the $R$-modules $C$ such that $\operatorname{Hom}_{R}(Q, C)=$ $0=\operatorname{Ext}_{R}^{1}(Q, C)$, where $Q$ is the field of fractions of $R$.

The category of Harrison's co-torsion abelian groups is an abelian subcategory in $\mathbb{Z}$-mod. So is the category of Matlis' cotorsion $R$-modules, provided that the $R$-module $Q$ has projective dimension 1 (see [20, p. 13] and [24, Theorem 3.4(a)]). Commutative domains $R$ with the latter property came to be known as Matlis domains [11, Section IV.4].

The modern terminology originates from Enochs' paper [9], where an $R$-module $C$ was called cotorsion if $\operatorname{Ext}_{R}^{1}(F, C)=0$ for all flat $R$-modules $F$. This definition, applicable to modules over an arbitrary associative ring, is now central to a vast theory, including two proofs [6] of Enochs' flat cover conjecture, the notion of a cotorsion theory (or cotorsion pair) (going back to Salce's paper [29]), and numerous examples and applications [13]. In particular, the pair of full subcategories (flat modules, cotorsion modules) in the category of left modules over an associative ring is a thematic example of complete cotorsion theory.

The following two definitions are due to Trlifaj [31, Section 2]. Let $Q$ denote the maximal ring of quotients of an associative $\operatorname{ring} R$. An $R$-module $C$ is called weakly cotorsion (or Matlis cotorsion) if $\operatorname{Ext}_{R}^{1}(Q, C)=0$. An $R$-module $F$ is called strongly flat if $\operatorname{Ext}_{R}^{1}(F, C)=0$ for all weakly cotorsion $R$-modules $C$. 
When $R$ is a commutative domain (so $Q$ is its field of fractions), every $R$-module has a weakly cotorsion envelope [31, Theorem 2.10(3)], [32, Example 3.2]. The problem of characterizing domains $R$ for which every $R$-module has a strongly flat cover was posed in the lecture notes [32, Section 5].

This problem was solved in the series of papers by Bazzoni and Salce $[4,5]$, where it was shown that, for a commutative domain $R$, all $R$-modules have strongly flat covers if and only if all flat modules are strongly flat, and if and only if $R$ is an almost perfect domain. The latter condition means that, for every nonzero element $r \in R$, the quotient ring $R / r R$ is perfect (in the sense of the classical paper of Bass [2]), that is, all flat modules over $R / r R$ are projective.

The following generalization of the results of Bazzoni-Salce was obtained in a recent work of Fuchs and Salce [12]. Let $R$ be a commutative ring and $Q$ be its classical ring of fractions. Then all flat $R$-modules are strongly flat if and only if $R$ is an almost perfect ring, which means that the quotient ring $R / r R$ is a perfect ring for every nonzero-divisor $r \in R$ and the ring of fractions $Q$ is a perfect ring. This result is the starting point for the present research.

1.2. Let $R$ be a commutative ring and $S \subset R$ be a multiplicative subset (which may contain some zero-divisors in $R$ ). Let us say that an $R$-module $C$ is $S$-weakly cotorsion if $\operatorname{Ext}_{R}^{1}\left(S^{-1} R, C\right)=0$, where $S^{-1} R$ denotes the localization of the ring $R$ at the multiplicative subset $S$. An $R$-module $F$ is said to be $S$-strongly flat if $\operatorname{Ext}_{R}^{1}(F, C)=0$ for all $S$-weakly cotorsion $R$-modules $C$.

Equivalently, an $R$-module $F$ is $S$-strongly flat if and only if it is a direct summand of an $R$-module $G$ for which there exists an exact sequence of $R$-modules

$$
0 \longrightarrow U \longrightarrow G \longrightarrow V \longrightarrow 0
$$

where $U$ is a free $R$-module and $V$ is a free $S^{-1} R$-module. This is a corollary of the general results about cotorsion theories generated by a set of objects in the category of modules over a ring [13, Corollary 6.13].

The first aim of this paper is to discuss the following

Optimistic Conjecture 1.1. Let $R$ be a commutative ring and $S \subset R$ be a multiplicative subset such that the projective dimension of the $R$-module $S^{-1} R$ does not exceed 1. Then a flat $R$-module $F$ is $S$-strongly flat if and only if the following two conditions hold: the $R / s R$-module $F / s F$ is projective for every element $s \in S$, and the $S^{-1} R$-module $S^{-1} F$ is projective.

Here the notation is $S^{-1} F=S^{-1} R \otimes_{R} F$. Notice that the "only if" assertion in Optimistic Conjecture 1.1 follows immediately from the description of strongly flat modules in terms of the exact sequence (1), as such a sequence remains exact after applying $R / s R \otimes_{R}-$, and the $R / s R$-module $V / s V$ vanishes for all $s \in S$, while $S^{-1} V=V$ is a free $S^{-1} R$-module.

It should be pointed out that the condition on the projective dimension of the $R$-module $S^{-1} R$ in Optimistic Conjecture 1.1 does indeed seem to be necessary. Indeed, let $H$ be the first syzygy module of the $R$-module $S^{-1} R$, i. e., the leftmost 
term of a short exact sequence of $R$-modules $0 \longrightarrow H \longrightarrow P \longrightarrow S^{-1} R \longrightarrow 0$ with a projective $R$-module $P$. Let us introduce the notation $\operatorname{pd}_{R} M$ for the projective dimension of an $R$-module $M$.

Proposition 1.2. Let $R$ be a commutative ring and $S \subset R$ be a multiplicative subset consisting of (some) nonzero-divisors in $R$. Assume that $\operatorname{pd}_{R} S^{-1} R \geq 2$, and let $H$ be the first syzygy module of the $R$-module $S^{-1} R$. Then the $R$-module $H$ is flat, the $R / s R$-module $H / s H$ is projective for every $s \in S$, and the $S^{-1} R$-module $S^{-1} H$ is projective, but the $R$-module $H$ is not $S$-strongly flat.

Let us now formulate the positive results that we can prove.

Theorem 1.3. Let $S$ be a countable multiplicative subset in a commutative ring $R$. Then a flat $R$-module $F$ is $S$-strongly flat if and only if the $R / s R$-module $F / s F$ is projective for every $s \in S$ and the $S^{-1} R$-module $S^{-1} F$ is projective.

Theorem 1.4. Let $R$ be a commutative ring and $S \subset R$ be a multiplicative subset consisting of (some) nonzero-divisors in $R$. Assume that the projective dimension of the $R$-module $S^{-1} R$ does not exceed 1 . Then a flat $R$-module $F$ is $S$-strongly flat if and only if the $R / s R$-module $F / s F$ is projective for every $s \in S$ and the $S^{-1} R$-module $S^{-1} F$ is projective.

Notice that one has $\operatorname{pd}_{R} S^{-1} R \leq 1$ for any countable multiplicative subset $S \subset R$, but the converse is not true. For example, if $R$ is a Noetherian commutative ring of Krull dimension 1, then the projective dimension of any flat $R$-module does not exceed 1 [28, Corollaire II.3.3.2] (cf. [23, Corollary 13.7(a)]).

Nevertheless, the following result is known $[10,1]$ : if $R$ is a commutative ring and $S \subset R$ is a multiplicative subset of nonzero-divisors, then the projective dimension of the $R$-module $S^{-1} R$ does not exceed 1 if and only if the $R$-module $S^{-1} R / R$ is a direct sum of countably generated modules. Our Theorem 1.4 is deduced from this result together with the same computations with countable multiplicative subsets $S \subset R$ which we use to prove Theorem 1.3.

Moreover, the following slight generalization of Theorem 1.4 is provable using the results of the papers $[10,1]$. Given a commutative ring $R$ and a multiplicative subset $S \subset R$, we will say that the $S$-torsion in $R$ is bounded if there exists an element $s_{0} \in S$ such that $s r=0$ for $s \in S$ and $r \in R$ implies $s_{0} r=0$.

Theorem 1.5. Let $R$ be a commutative ring and $S \subset R$ be a multiplicative subset such that the $S$-torsion in $R$ is bounded. Assume that the projective dimension of the $R$-module $S^{-1} R$ does not exceed 1 . Then a flat $R$-module $F$ is $S$-strongly flat if and only if the $R / s R$-module $F / s F$ is projective for every $s \in S$ and the $S^{-1} R$-module $S^{-1} F$ is projective.

Notice that Theorem 1.3 is not a particular case of Theorem 1.5 as, for a countable multiplicative subset $S$ in a commutative ring $R$, the $S$-torsion in $R$ does not need to be bounded.

Finally, let us point out that yet another particular case of Optimistic Conjecture 1.1 is shown to be true in the paper [3]. If $R$ is an $S$-h-nil commutative ring, 
that is, for every element $s \in S$ the $\operatorname{ring} R / s R$ is semilocal of Krull dimension 0, then the projective dimension of the $R$-module $S^{-1} R$ does not exceed 1 [3, Corollary 6.13], and the assertion of our Optimistic Conjecture 1.1 holds for the multiplicative subset $S$ in the ring $R$ [3, Proposition 7.13].

1.3. Let us say a few words about our proofs of Theorems 1.3-1.5. Nothing (or almost nothing) is being done, in the course of these proofs, with a flat $R$-module $F$ satisfying the conditions of these theorems. Instead, we work with an arbitrary $S$-weakly cotorsion $R$-module $C$, proving that it can be obtained, using a certain set of rules or operations, from $R$-modules of simpler nature.

Classically, the theory of cotorsion pairs [29, 13] is developed as the theory of Ext ${ }^{1}$-orthogonal classes of modules. Given any class of modules over an associative ring $R$, its left $\operatorname{Ext}_{R}^{1}$-orthogonal class is closed under transfinitely iterated extesions (in the sense of the inductive limit) [8, Lemma 1] and direct summands, while its right Ext $_{R}^{1}$-orthogonal class is closed under transfinitely iterated extensions (in the sense of the projective limit) [8, Proposition 18] and direct summands. In the important particular case of a hereditary cotorsion pair, the Ext ${ }^{1}$-orthogonal class coincides with the Ext ${ }^{\geq 1}$-orthogonal class.

We change the point of view slightly and assign to a class of $R$-modules two right orthogonal classes: the Ext ${ }^{\geq 1}$-orthogonal class and the Ext ${ }^{\geq 2}$-orthogonal class. In addition to the closedness with respect to transfinitely iterated extensions (in the sense of the projective limit), the pair of classes of modules so obtained has certain properties of closedness with respect to kernels of surjections and cokernels of injections. Abstracting from these properties, we define the notion of right 1-obtainability of an $R$-module from a given "seed class" of $R$-modules.

Proposition 1.6. Let $S$ be a countable multiplicative subset in a commutative ring $R$. Then an $R$-module is $S$-weakly cotorsion if and only if it is right 1-obtainable from $R / s R$-modules, $s \in S$, and $S^{-1} R$-modules.

Proposition 1.7. Let $R$ be a commutative ring and $S \subset R$ be a multiplicative subset consisting of (some) nonzero-divisors in $R$. Assume that the projective dimension of the $R$-module $S^{-1} R$ does not exceed 1 . Then an $R$-module is $S$-weakly cotorsion if and only if it is right 1-obtainable from $R / s R$-modules, $s \in S$, and $S^{-1} R$-modules.

Proposition 1.8. Let $R$ be a commutative ring and $S \subset R$ be a multiplicative subset such that the $S$-torsion in $R$ is bounded. Assume that the projective dimension of the $R$-module $S^{-1} R$ does not exceed 1 . Then an $R$-module is $S$-weakly cotorsion if and only if it is right 1-obtainable from $R / s R$-modules, $s \in S$, and $S^{-1} R$-modules.

In this paper, Theorems 1.3-1.5 are (easily) deduced from Propositions 1.6-1.8, while the proofs of the latter require some substantial work.

1.4. Let us consider the following generalization of the setting of Sections 1.2-1.3. Let $S_{1}, \ldots, S_{m} \subset R$ be a finite collection of multiplicative subsets in $R$. We will denote the collection $\left\{S_{1}, \ldots, S_{m}\right\}$ by the single letter $\mathbf{S}$ for brevity. Let us say that an $R$-module $C$ is $\mathbf{S}$-weakly cotorsion if $\operatorname{Ext}_{R}^{1}\left(S_{j}^{-1} R, C\right)=0$ for all $j=1, \ldots, m$. An 
$R$-module $F$ is said to be $\mathbf{S}$-strongly flat if $\operatorname{Ext}_{R}^{1}(F, C)=0$ for all $\mathbf{S}$-weakly cotorsion $R$-modules $C$. Equivalently, an $R$-module $F$ is $\mathbf{S}$-strongly flat if and only if it is a direct summand of a transfinitely iterated extension, in the sense of the inductive limit, of $R$-modules isomorphic to $R$ or $S_{j}^{-1} R, 1 \leq j \leq m$ [13, Corollary 6.14].

Given two multiplicative subsets $S$ and $T \subset R$, we denote by $S T \subset R$ the multiplicative subset generated by $S$ and $T$ in $R$; and similarly, given a finite collection of multiplicative subsets $\left\{S_{j}\right\}$ in $R$, we denote by $\prod_{j} S_{j} \subset R$ the multiplicative subset generated by them. For any subset of indices $J \subset\{1, \ldots, m\}$, denote by $S_{J} \subset R$ the multiplicative subset $\prod_{j \in J} S_{j} \subset R$. Given a finite collection of multiplicative subsets $\mathbf{S}=\left\{S_{j} \mid 1 \leq j \leq m\right\}$ in a commutative ring $R$, we denote by $\mathbf{S}^{\times}$the finite collection of multiplicative subsets $\left\{S_{J} \mid J \subset\{1, \ldots, m\}\right\}$.

Let $J \subset\{1, \ldots, m\}$ be a subset of indices; denote by $K$ the complementary subset $K=\{1, \ldots, m\} \backslash J$. Given a collection of multiplicative subsets $\mathbf{S}=\left\{S_{1}, \ldots, S_{m}\right\}$ in a commutative ring $R$ and a subset of indices $K \subset\{1, \ldots, m\}$, denote by a single letter $\mathbf{s}$ a collection of elements $\left(s_{k} \in S_{k}\right)_{k \in K}$. Let $R_{J, \mathbf{s}}$ denote the quotient ring of the ring $S_{J}^{-1} R$ by the ideal generated by all the elements $s_{k} \in S_{J}^{-1} R, k \in K$. So the ring $R_{J, \mathbf{s}}$ is the result of inverting all the multiplicative subsets $S_{j} \subset R, j \in J$, and annihilating all the elements $s_{k} \in R, k \in K$, in the ring $R$.

The following formulation is the analogue of Optimistic Conjecture 1.1 for several multiplicative subsets.

Optimistic Conjecture 1.9. Let $R$ be a commutative ring and $S_{1}, \ldots, S_{m} \subset R$ be a finite collection of multiplicative subsets in $R$. Assume that, for every subset of indices $J \subset\{1, \ldots, m\}$, the projective dimension of the $R$-module $S_{J}^{-1} R$ does not exceed 1. Then a flat $R$-module $F$ is $\mathbf{S}^{\times}$-strongly flat if and only if the $R_{J, \mathbf{s}}$-module $R_{J, \mathbf{s}} \otimes_{R} F$ is projective for every subset of indices $J \subset\{1, \ldots, m\}$ and any choice of elements $s_{k} \in S_{k}, \quad k \in\{1, \ldots, m\} \backslash J$.

Here is the theorem that we can actually prove.

Theorem 1.10. Let $S_{1}, \ldots, S_{m} \subset R$ be a finite collection of multiplicative subsets in a commutative ring $R$. Assume that, for every subset of indices $J \subset\{1, \ldots, m\}$, the projective dimension of the $R$-module $S_{J}^{-1} R$ does not exceed 1 . Furthermore, assume that for every $j=1, \ldots, m$, one of the two possibilities is realized: either $S_{j}$ is countable, or the $S_{j}$-torsion in $R$ is bounded. Then a flat $R$-module $F$ is $\mathbf{S}^{\times}$-strongly flat if and only if the $R_{J, \mathbf{s}}$-module $R_{J, \mathbf{s}} \otimes_{R} F$ is projective for every subset of indices $J \subset\{1, \ldots, m\}$ and any choice of elements $s_{k} \in S_{k}, k \in\{1, \ldots, m\} \backslash J$.

We deduce Theorem 1.10 from the following proposition.

Proposition 1.11. Let $S_{1}, \ldots, S_{m} \subset R$ be a finite collection of multiplicative subsets in a commutative ring $R$. Assume that, for every $J \subset\{1, \ldots, m\}$, the projective dimension of the $R$-module $S_{J}^{-1} R$ does not exceed 1 . Furthermore, assume that, for every $1 \leq j \leq m$, either $S_{j}$ is countable, or the $S_{j}$-torsion in $R$ is bounded. Then an $R$-module is $\mathbf{S}^{\times}$-weakly cotorsion if and only if it is right 1-obtainable from the class of all $R_{J, \mathbf{s}}$-modules (viewed as $R$-modules via the restriction of scalars), where 
$J$ runs over all the subsets of indices $J \subset\{1, \ldots, m\}$ and $\mathbf{s}$ runs over all the choices of elements $s_{k} \in S_{k}, k \in\{1, \ldots, m\} \backslash J$.

Of course, the product $S T$ of any two countable multiplicative subsets $S, T \subset R$ is countable; and one easily observes that, for any two multiplicative subsets $S, T \subset R$, the $S T$-torsion in $R$ bounded whenever the $S$-torsion is bounded and $T$-torsion is bounded. But the product of two multiplicative subsets, one of which is countable and the other has bounded torsion, may satisfy neither condition.

On the other hand, if all the multiplicative subsets $S_{1}, \ldots, S_{m} \subset R$ are countable, then the condition on the projective dimension of the $R$-modules $S_{J}^{-1} R$ in Theorem 1.10 and Proposition 1.11 is satisfied automatically.

1.5. Now let us formulate our descriptions of flat and Enochs cotorsion $R$-modules. The following two results were obtained in the paper [23, Section 13] (see [23, Corollary 13.11 and Theorem 13.9(b)]).

Theorem 1.12. Let $R$ be a Noetherian commutative ring with finite spectrum. Then there exists an element $s \in R$ such that, denoting by $S$ the multiplicative subset $\left\{1, s, s^{2}, s^{3}, \ldots\right\} \subset R$ generated by $s$, all flat $R$-modules are $S$-strongly flat.

Theorem 1.13. Let $R$ be a Noetherian commutative ring of Krull dimension 1 . Then there exists a multiplicative subset $S \subset R$ such that all flat $R$-modules are $S$-strongly flat.

In the rest of this introduction we discuss generalizations of these theorems to more complicated Noetherian commutative rings.

The following simple example is the starting point. Let $P$ be a principal ideal domain (PID). The content of a polynomial $p(x)=p_{n} x^{n}+p_{n-1} x^{n-1}+\cdots+p_{0} \in P[x]$ is defined as the greatest common divisor of its coefficients $p_{n}, \ldots, p_{0}$. So the content of a polynomial in one variable $x$ over the ring $P$ is an element of $P$ defined up to a multiplication by an invertible element of $P$. By Gauss Lemma, the content of the product of two polynomials is equal to the product of their contents.

Denote by $S_{1} \subset P[x]$ the multiplicative subset of all nonzero elements of $P$, viewed as elements of $P[x]$, and by $S_{2} \subset P[x]$ the multiplicative subset of all polynomials with content 1. Set $\mathbf{S}=\left\{S_{1}, S_{2}\right\}$ to be the collection of two multiplicative subsets $S_{1}$ and $S_{2}$ in the ring $R=P[x]$.

Theorem 1.14. For any countable (commutative) principal ideal domain $P$, all flat $P[x]$-modules are $\mathbf{S}^{\times}$-strongly flat for the above collection of two multiplicative subsets $\mathbf{S}=\left\{S_{1}, S_{2}\right\}$ in $R=P[x]$.

The result of Theorem 1.14 generalizes to Noetherian rings of Krull dimension 2 as follows.

Theorem 1.15. Let $R$ be a Noetherian commutative ring of Krull dimension 2 with countable spectrum. Then there exists a pair of countable multiplicative subsets $S_{1}$ and $S_{2} \subset R$ such that all flat $R$-modules are $\mathbf{S}^{\times}$-strongly flat for $\mathbf{S}=\left\{S_{1}, S_{2}\right\}$. 
In other words, the assertions of Theorems 1.14 and 1.15 mean that, in their respective assumptions, every flat $R$-module is a direct summand of a transfinitely iterated extension, in the sense of the inductive limit, of $R$-modules isomorphic to $R$, $S_{1}^{-1} R, S_{2}^{-1} R$, or $\left(S_{1} S_{2}\right)^{-1} R$.

Furthermore, the construction of Theorem 1.15 generalizes to higher Krull dimensions $d$ in the following way. Define a function $\mu: \mathbb{Z}_{\geq 0} \longrightarrow \mathbb{Z}_{\geq 0}$ by the rule

$$
\mu(d)=d+(d-2)+(d-4)+\cdots=\sum_{i \in \mathbb{Z}}^{0 \leq 2 i \leq d} d-2 i,
$$

or equivalently, $\mu(d)$ is the closest integer to $(d+1)^{2} / 4$. So we have $\mu(0)=0$, $\mu(1)=1, \mu(2)=2, \mu(3)=4, \mu(4)=6$, etc.

Theorem 1.16. Let $R$ be a Noetherian commutative ring of finite Krull dimensiond with countable spectrum. Then there exists a collection of $m=\mu(d)$ countable multiplicative subsets $S_{1}, \ldots, S_{m} \subset R$ such that all flat $R$-modules are $\mathbf{S}^{\times}$-strongly flat for $\mathbf{S}=\left\{S_{1}, \ldots, S_{m}\right\}$.

In all the three Theorems $1.14,1.15$, and 1.16, the multiplicative subsets $S_{1}, \ldots$, $S_{m} \subset R$ are constructed in such a way that all the rings $R_{J, \mathbf{s}}$ (in the notation of Section 1.4) are Artinian. Then all flat $R_{J, \mathbf{s}}$-modules are projective, and the assertion that all flat $R$-modules are $\mathbf{S}^{\times}$-strongly flat follows from Theorem 1.10.

1.6. The following approach allows to generalize Theorem 1.16 even further by getting rid of the finite Krull dimension assumption on the ring $R$. Besides, it allows to obtain some results applicable to Noetherian rings with uncountable spectrum.

Let $R$ be a commutative ring. Let us say that an $R$-module $C$ is almost cotorsion if $\operatorname{Ext}_{R}^{1}\left(S^{-1} R, C\right)=0$ for all (at most) countable multiplicative subsets $S \subset R$. We say that an $R$-module $F$ is quite flat if $\operatorname{Ext}_{R}^{1}(F, C)=0$ for all almost cotorsion $R$-modules $C$. Equivalently, an $R$-module $F$ is quite flat if and only if it is a direct summand of a transfinitely iterated extension, in the sense of the inductive limit, of $R$-modules isomorphic to $S^{-1} R$, where $S \subset R$ are countable multiplicative subsets.

Theorem 1.17. Let $R$ be a Noetherian commutative ring with countable spectrum. Then all flat $R$-modules are quite flat.

Notice that, in an (infinite) Noetherian ring, the cardinality of the set of all ideals does not exceed the cardinality of the ring. Thus any countable Noetherian ring has at most countable spectrum (while the converse, of course, does not hold).

Theorem 1.17 can be deduced from the following main lemma (which does not assume countability of the spectrum).

Main Lemma 1.18. Let $R$ be a Noetherian commutative ring and $S \subset R$ be a countable multiplicative subset. Then a flat $R$-module $F$ is quite flat if and only if the $R / s R$-module $F / s F$ is quite flat for all $s \in S$ and the $S^{-1} R$-module $S^{-1} F$ is quite flat.

Alternatively, Theorem 1.17 can be deduced from the following proposition. 
Proposition 1.19. Let $R$ be a Noetherian commutative ring with countable spectrum. Then an $R$-module is almost cotorsion if and only if it is right 1-obtainable from vector spaces over the residue fields of prime ideals in $R$ (viewed as $R$-modules via the restriction of scalars).

One observes that all vector spaces over fields are (Enochs) cotorsion, all restrictions of scalars take cotorsion modules to cotorsion modules, and all modules right 1-obtainable from cotorsion modules are cotorsion. Thus Proposition 1.19 implies (or, if one wishes, is equivalent to the combination of) the following two corollaries.

Corollary 1.20. Let $R$ be a Noetherian commutative ring with countable spectrum. Then all almost cotorsion $R$-modules are cotorsion.

Corollary 1.21. Let $R$ be a Noetherian commutative ring with countable spectrum. Then an R-module is (Enochs) cotorsion if and only if it is right 1-obtainable from vector spaces over the residue fields of prime ideals in $R$ (viewed as $R$-modules via the restriction of scalars).

Corollary 1.20 is, of course, just a restatement of Theorem 1.17, while Corollary 1.21 provides a description of cotorsion modules over a Noetherian commutative ring with countable spectrum. Notice that a description of flat cotorsion modules over a Noetherian commutative ring, obtained by Enochs in [9], plays in important role in the locally cotorsion contraherent cosheaf theory [22, Section 5.1]. A description of cotorsion modules over a Noetherian commutative ring of Krull dimension 1 was obtained in [23, Corollary 13.12].

Both Main Lemma 1.18 and Proposition 1.19 can be deduced from the following stronger version of the main lemma (which, once again, does not assume countability of the spectrum).

Main Lemma 1.22. Let $R$ be a Noetherian commutative ring and $S \subset R$ be a countable multiplicative subset. Then an $R$-module is almost cotorsion if and only if it is right 1-obtainable from almost cotorsion $R / s R$-modules, $s \in S$, and almost cotorsion $S^{-1} R$-modules.

1.7. Finally, we should mention that both Main Lemmas 1.18 and 1.22 are generalizable to some situations involving non-Noetherian commutative rings. Notice that, for any multiplicative subset $S$ in a Noetherian commutative ring $R$, the $S$-torsion in $R$ is necessarily bounded.

Main Lemma 1.23. Let $R$ be a commutative ring and $S \subset R$ be a countable multiplicative subset such that the $S$-torsion in $R$ is bounded. Then a flat $R$-module $F$ is quite flat if and only if the $R / s R$-module $F / s F$ is quite flat for all $s \in S$ and the $S^{-1} R$-module $S^{-1} F$ is quite flat.

Main Lemma 1.24. Let $R$ be a commutative ring and $S \subset R$ be a countable multiplicative subset such that the $S$-torsion in $R$ is bounded. Then an $R$-module is almost cotorsion if and only if it is right 1-obtainable from almost cotorsion $R / s R$-modules, $s \in S$, and almost cotorsion $S^{-1} R$-modules. 
Here, as with the Noetherian versions of the main lemmas (and as with the resuls of this paper generally), the description of (quite) flat modules follows easily from the description of (almost) cotorsion ones, i. e., Main Lemma 1.23 is deduced from Main Lemma 1.24.

1.8. This paper is a sequel to the paper [27], where largely the same techniques are applied to a number of similar problems. The main difference is that the exposition in [27] is restricted to multiplicative subsets $S \subset R$ generated by a single element, $S=\left\{1, r, r^{2}, r^{3}, \ldots\right\}$, where $r \in R$ (as these are important for the algebro-geometric applications to which [27] aims). Thus many results of this paper are generalizations of the respective results of [27]. In particular, the technique of "obtainable modules" first appeared in [27, Sections 3 and 6].

The reader may wish to glance into [27, Section 1] for an overview of the results and an outline of the arguments in [27] relevant in the context of the present paper (the requisite definitions can be found in [27, Section 0.5]).

In particular, [27, Toy Main Lemma 1.8] is notable as the simplest version of Theorem 1.3 (while [27, Lemma 1.10] is an even simpler result whose proof is dual in some sense). [27, Toy Main Proposition 4.8] is a particular case of Proposition 1.6, while [27, Theorem 1.9] is a particular case of Theorem 1.10 and [27, Main Proposition 8.1] is a particular case of Proposition 1.11.

[27, Main Lemma 1.4] is somewhat similar to (but simpler than) Main Lemma 1.18, while [27, Main Proposition 7.1] is somewhat similar to (but simpler than) Main Lemma 1.22. Finally, [27, Main Lemma 1.7] is somewhat similar to (but simpler than) Main Lemma 1.23, while [27, Main Proposition 7.2] is somewhat similar to (but simpler than) Main Lemma 1.24.

1.9. The proofs of Proposition 1.2, Theorems 1.3-1.5, and Propositions 1.6-1.8 are presented in Section 4. The proofs of Theorem 1.10 and Proposition 1.11 are given in Section 5. Theorems 1.14-1.16 are proved in Section 6. The proofs of Theorem 1.17, Proposition 1.19, Main Lemmas 1.18 and 1.22-1.24, and Corollaries 1.20-1.21 are contained in the last Section 8.

Acknowledgement. The first author is grateful to Silvana Bazzoni for very helpful discussions and communications. This paper grew out of the first author's visits to Padova and Prague, and he wishes to thank Silvana Bazzoni and Jan Trlifaj for the kind invitations. We also wish to thank Jan Trlifaj for very helpful discussions. The first author's research is supported by the Israel Science Foundation grant \# 446/15 and by the Grant Agency of the Czech Republic under the grant P201/12/G028. The second author's research is supported by the Grant Agency of the Czech Republic under the grant 17-23112S and by the SVV project under the grant SVV-2017-260456.

\section{Obtainable Modules}

In this section, $R$ is an associative ring and $R$-mod is the abelian category of left $R$-modules. The exposition below largely follows [27, Sections 3 and 6$]$. 
Given a class of objects (full subcategory) $\mathrm{F} \subset R$-mod, we denote by $\mathrm{F}^{\perp_{1}} \subset R$-mod the class of all objects $C \in R$-mod such that $\operatorname{Ext}_{R}^{1}(F, C)=0$ for all $F \in \mathrm{F}$. Similarly, for any integer $n \geq 0$, we denote by $\mathrm{F}^{\perp \geq n} \subset R$-mod the class of all objects $C \in R$-mod such that $\operatorname{Ext}_{R}^{i}(F, C)=0$ for all $F \in \mathrm{F}$ and $i \geq n$.

Let us also introduce notation for the dual operations on classes of modules. Given a class of objects $\mathrm{E} \subset R$-mod, we denote by ${ }^{{ }_{1}} \mathrm{E} \subset R$-mod the class of all objects $F \in R$-mod such that $\operatorname{Ext}_{R}^{1}(F, E)=0$ for all $E \in \mathrm{E}$. For any integer $n \geq 0$, we denote by ${ }^{{ }_{2} n} \mathrm{E} \subset R$-mod the class of all objects $F \in R$-mod such that $\operatorname{Ext}_{R}^{i}(F, E)=0$ for all $E \in \mathrm{E}$ and all $i \geq n$.

Now let us define transfinitely iterated extensions. Let $F$ be a left $R$-module and $\gamma$ be an ordinal. Suppose that for every ordinal $\alpha \leq \gamma$ we are given an $R$-submodule $F_{\alpha} \subset F$ such that the following conditions are satisfied:

- $F_{0}=0$ and $F_{\gamma}=F$;

- one has $F_{\alpha} \subset F_{\beta}$ for all $\alpha \leq \beta \leq \gamma$;

- and one has $F_{\beta}=\bigcup_{\alpha<\beta} F_{\alpha}$ for all limit ordinals $\beta \leq \gamma$.

Then one says that the $R$-module $F$ is a transfinitely iterated extension (in the sense of the inductive limit) of the $R$-modules $F_{\alpha+1} / F_{\alpha}$, where $0 \leq \alpha<\gamma$.

Here is the dual definition. Let $G$ be a left $R$-module and $\delta$ be an ordinal. Suppose that for every ordinal $\alpha \leq \delta$ we are given a left $R$-module $G_{\alpha}$ and for every pair of ordinals $\alpha<\beta \leq \delta$ there is an $R$-module morphism $G_{\beta} \longrightarrow G_{\alpha}$ such that the following conditions hold:

- $G_{0}=0$ and $G_{\delta}=G$;

- the triangle diagrams $G_{\gamma} \longrightarrow G_{\beta} \longrightarrow G_{\alpha}$ are commutative for all triples of ordinals $\alpha<\beta<\gamma \leq \delta$;

- the induced morphism into the projective limit $G_{\beta} \longrightarrow \lim _{\alpha<\beta} G_{\alpha}$ is an isomorphism for all limit ordinals $\beta \leq \delta$;

- the morphism $G_{\alpha+1} \longrightarrow G_{\alpha}$ is surjective for all $\alpha<\delta$.

Denote by $H_{\alpha}$ the kernel of the morphism $G_{\alpha+1} \longrightarrow G_{\alpha}$. Then we will say that the left $R$-module $G$ is a transfinitely iterated exension (in the sense of the projective limit) of the left $R$-modules $H_{\alpha}$, where $0 \leq \alpha<\delta$.

Lemma 2.1. (a) Let $\mathrm{E} \subset R$-mod be a class of left $R$-modules. Then the class of left $R$-modules $\mathrm{F}={ }^{\perp_{1}} \mathrm{E}$ is closed under transfinitely iterated extensions, in the sense of the inductive limit, and direct summands.

(b) Let $\mathrm{F} \subset R$-mod be a class of left $R$-modules. Then the class of left $R$-modules $\mathrm{C}=\mathrm{F}^{\perp_{1}}$ is closed under transfinitely iterated extensions, in the sense of the projective limit, and direct summands.

Proof. Closedness under direct summands is obvious. The assertions about closedness with respect to transfinitely iterated extensions are known as the Eklof Lemma. Part (a) is the classical version of the Eklof Lemma [8, Lemma 1], while part (b) is the dual version [8, Proposition 18]. 
Lemma 2.2. (a) Let $\mathrm{E} \subset R$-mod be a class of left $R$-modules and $n \geq 1$ be an integer. Then the class of left $R$-modules $\mathrm{F}={ }^{\perp_{\geq n}} \mathrm{E}$ is closed under direct summands, extensions, kernels of surjective morphisms, infinite direct sums, and transfinitely iterated extensions in the sense of the inductive limit.

(b) Let $\mathrm{F} \subset R$-mod be a class of left $R$-modules and $n \geq 1$ be an integer. Then the class of left $R$-modules $\mathrm{C}=\mathrm{F}^{\perp \geq n}$ is closed under direct summands, extensions, cokernels of injective morphisms, infinite products, and transfinitely iterated extensions in the sense of the projective limit.

Proof. Notice that all the operations listed in part (a), with the exception of direct summands and kernels of surjective morphisms, are particular cases of transfinitely iterated extensions in the sense of the inductive limit, and similarly, all the operations listed in part (b), with the exception of direct summands and cokernels of injective morphisms, are particular cases of transfinitely iterated extensions in the sense of the projective limit. The closedness properties of the classes of modules in Lemma 2.2 with respect to transfinitely iterated extensions follow from the similar properties of the classes of modules in Lemma 2.1, while closedness with respect to kernels of surjections or cokernels of injections is easily checked using the long exact sequence of $\operatorname{Ext}_{R}^{*}$. We refer to [27, proofs of Lemmas 3.2 and 6.1] for the details.

Let $\mathrm{F} \subset R$-mod be a class of left $R$-modules. For every $n \geq 1$, consider the class of left $R$-modules $\mathrm{C}_{n}=\mathrm{F}^{\perp \geq n}$.

Lemma 2.3. For any class of objects $\mathrm{F} \subset R$-mod, the classes of objects $\mathrm{C}_{n}=\mathrm{F}^{\perp \geq n}$ have the following properties:

(i) for any $n \geq 1$, one has $\mathrm{C}_{n} \subset \mathrm{C}_{n+1}$;

(ii) for any $n \geq 1$, the class of objects $C_{n} \subset R$-mod is closed under direct summands, extensions, cokernels of injective morphisms, infinite products, and transfinitely iterated extensions in the sense of the projective limit;

(iii) the kernel of any surjective morphism from an object of $\mathrm{C}_{n+1}$ to an object of $\mathrm{C}_{n}$ belongs to $\mathrm{C}_{n+1}$;

(iv) the cokernel of any injective morphism from an object of $\mathrm{C}_{n+1}$ to an object of $\mathrm{C}_{n}$ belongs to $\mathrm{C}_{n}$.

Proof. This is [27, Lemma 6.1]. The property (i) holds by the definition, (ii) follows from Lemma 2.2(b), and (iii-iv) are easily provable using the long exact sequence of $\operatorname{Ext}_{R}^{*}$.

Let $\mathrm{E} \subset R$-mod be a fixed class of objects. Set $\mathrm{F}={ }^{\perp_{\geq 1}} \mathrm{E}$ and $\mathrm{C}=\mathrm{F}^{\perp_{\geq n}}$ for $n=1$ and 2. Our aim is to formulate a technique that would potentially allow to describe the class $C_{1}=\left({ }^{{ }^{2}} \mathrm{E}\right)^{\perp \geq 1}$, at least in some special situations. The following two definitions can be found in [27, Definition 3.3 and 6.2].

We will say that an object $C \in R$-mod is simply right obtainable from a class $\mathrm{E} \subset R$-mod if $C$ belongs to the (obviously, unique) minimal class of objects in $R$-mod containing $\mathrm{E}$ and closed under direct summands, extensions, cokernels of 
injective morphisms, infinite products, and transfinitely iterated extensions in the sense of the projective limit.

Furthermore, the pair of classes of objects right 1-obtainable from $\mathrm{E}$ and right 2-obtainable from $\mathrm{E}$ is defined as the (obviously, unique) minimal pair of classes of objects in $R$-mod satisfying the following generation rules:

(i) all the objects of $\mathrm{E}$ are right 1-obtainable; all the right 1-obtainable objects are right 2-obtainable;

(ii) all the objects simply right obtainable from right $n$-obtainable objects are right $n$-obtainable (for $n=1$ or 2 );

(iii) the kernel of any surjective morphism from a right 2-obtainable object to a right 1-obtainable object is right 2-obtainable;

(iv) the cokernel of any injective morphism from a right 2-obtainable object to a right 1-obtainable object is right 1-obtainable.

Lemma 2.4. For any class of objects $\mathrm{E} \subset R$-mod, all the objects right 1-obtainable from $\mathrm{E}$ belong to the class $\mathrm{C}_{1}=\left({ }^{\perp_{\geq 1}} \mathrm{E}\right)^{\perp \geq 1} \subset R$-mod. All the objects right 2-obtainable from $\mathrm{E}$ belong to the class $\mathrm{C}_{2}=\left({ }^{\perp_{\geq 1}} \mathrm{E}\right)^{\perp_{\geq 2}} \subset R$-mod.

Proof. This is [27, Lemma 6.3]. Both the assertions follow from Lemma 2.3.

Remark 2.5. We will never use the full power of the above definitions of obtainability in our constructions in this paper. In particular, all our transfinitely iterated extensions (in the sense of the projective limit) will be indexed by the ordinal of nonnegative integers $\omega$ (we will call these infinitely iterated extensions). However, we will sometimes use uncountably infinite products. Besides, the second part of the rule (i) and the rule (iii) will be only used in the following weak combination:

(iii') the kernel of any surjective morphism from a right 1-obtainable object to a right 1-obtainable object is right 2-obtainable.

We refer to [27, Remarks 3.7 and 6.4] for comparison.

In fact, the following lemma essentially captures all our uses of the rule (iii') (and hence also of the rules (i) and (iii)).

Lemma 2.6. Let $\mathrm{C}$ denote the class of all left $R$-modules $C$ such that $\operatorname{Ext}_{R}^{1}(Q, C)=0$ for all left $R$-modules $Q$ of projective dimension 1 . Then all left $R$-modules are right 2-obtainable from $\mathrm{C}$.

Proof. Any left $R$-module $A$ can be embedded into an $R$-module $B$ belonging to $C$ (e. g., an injective left $R$-module $B$ ). The quotient $R$-module $B / A$ then also belongs to $C$. Now $A$ is the kernel of the surjective morphism $B \longrightarrow B / A$, so it is right 2-obtainable from $B$ and $B / A$. 


\section{S-Contramodule $R$-Modules}

Let $R$ be a commutative ring. We will use the notation $\operatorname{pd}_{R} M$ for the projective dimension of an $R$-module $M$.

Let $S \subset R$ be a multiplicative subset. We will use the notation $S^{-1} R$ for the localization of the $\operatorname{ring} R$ at the multiplicative subset $S$. For any $R$-module $M$, we set $S^{-1} M=S^{-1} R \otimes_{R} M$.

An $R$-module $M$ is said to be $S$-torsion if for every element $x \in M$ there exists an element $s \in S$ such that $s x=0$ in $M$. The (unique) maximal $S$-torsion submodule of an $R$-module $M$ is denoted by $\Gamma_{S}(M) \subset M$. An $R$-module $M$ is said to have bounded $S$-torsion if there exists an element $s_{0} \in S$ such that $s_{0} \Gamma_{S}(M)=0$.

Assume that the projective dimension $\operatorname{pd}_{R} S^{-1} R$ of the $R$-module $S^{-1} R$ does not exceed 1. In this case, an $R$-module $C$ is said to be an $S$-contramodule [24] if $\operatorname{Hom}_{R}\left(S^{-1} R, C\right)=0=\operatorname{Ext}_{R}^{1}\left(S^{-1} R, C\right)$. The full subcategory of $S$-contramodule $R$-modules is denoted by $R-\bmod _{S \text {-ctra }} \subset R$-mod.

Given a complex of $R$-modules $K^{\bullet}$ and an $R$-module $A$, we will use the simplified notation $\operatorname{Ext}_{R}^{i}\left(K^{\bullet}, A\right)$ for the $R$-modules $\operatorname{Hom}_{\mathrm{D}(R \text {-mod })}\left(K^{\bullet}, A[i]\right)$ of morphisms in the derived category of $R$-modules $\mathrm{D}(R-\bmod )$.

The two-term complex of $R$-modules $R \longrightarrow S^{-1} R$ will be particularly important for us. We denote it by $K_{R, S}^{\bullet}$ and place in the cohomological degrees -1 and 0 (so one has $K_{R, S}^{-1}=R$ and $\left.K_{R, S}^{0}=S^{-1} R\right)$. The functor $A \longmapsto \operatorname{Ext}_{R}^{1}\left(K_{R, S}^{\bullet}, A\right)$ on the category of $R$-modules is denoted by $\Delta_{R, S}$.

Lemma 3.1. Let $R$ be a commutative ring and $S \subset R$ be a multiplicative subset such that $\operatorname{pd}_{R} S^{-1} R \leq 1$. Then

(a) the full subcategory $R$ - $\bmod _{S \text {-ctra }}$ is closed under kernels, cokernels, extensions, and infinite products in $R$-mod; so, in particular, $R$ - $\bmod _{S-c t r a}$ is an abelian category and its embedding $R-\bmod _{S \text {-ctra }} \longrightarrow R$-mod is an exact functor;

(b) for any $R$-module $A$, the $R$-module $\Delta_{R, S}(A)=\operatorname{Ext}_{R}^{1}\left(K_{R, S}^{\bullet}, A\right)$ is an $S$-contramodule; the functor $\Delta_{R, S}: R$-mod $\longrightarrow R-\bmod _{S \text {-ctra }}$ is left adjoint to the embedding functor $R$ - $\bmod _{S \text {-ctra }} \longrightarrow R$-mod.

Proof. This is [24, Theorem 3.4].

Let us also introduce notation for the $S$-completion functor $\Lambda_{R, S}: R$-mod $\longrightarrow$ $R-\bmod$

$$
\Lambda_{R, S}(A)=\lim _{s \in S} A / s A,
$$

where the projective limit is taken over the set $S$ endowed with the preorder $t \geq s$, $s, t \in S$ if there exists $r \in R$ such that $t=r s$. For any $R$-module $A$, there is a natural $R$-module morphism $\beta_{R, S, A}: \Delta_{R, S}(A) \longrightarrow \Lambda_{R, S}(A)$ [24, Lemma 2.1(b)].

The rest of this section is devoted to the study of right obtainability properties of $S$-contramodule $R$-modules (in the sense of Section 2). We consider three situations (corresponding to the assumption sets of three Theorems 1.3, 1.4, and 1.5) separately. 
3.1. Countable multiplicative subsets. Let $R$ be a commutative ring and $S \subset R$ be an (at most) countable multiplicative subset. Firstly we observe that the projective dimension of the $R$-module $S^{-1} R$ does not exceed 1 in this case [24, Lemma 1.9].

Let $s_{1}, s_{2}, s_{2}, \ldots$ be a sequence of elements of $S$ such that every element of $S$ appears infinitely many times in this sequence. Set $t_{0}=1$ and $t_{n}=s_{1} \cdots s_{n}$ for all $n \geq 1$. Then for any $R$-module $A$ we have $\Lambda_{R, S}(A)=\lim _{n \geq 1} A / t_{n} A$, where the projective limit is taken over the natural surjective maps $A / t_{n} A \longrightarrow A / t_{n-1} A$.

For any $R$-module $A$ and an element $r \in R$, denote by ${ }_{r} A \subset A$ the submodule of all elements annihilated by $r$ in $A$ (i. e., the kernel of the multiplication map $r: A \longrightarrow A)$. Then the $R$-modules $t_{n} A, n \geq 1$ form a projective system with the projection maps $s_{n}:{ }_{t_{n}} A \longrightarrow t_{t_{n-1}} A$.

Lemma 3.2. Let $R$ be a commutative ring and $S \subset R$ be an (at most) countable multiplicative subset. Then for any $R$-module $A$ there is a natural short exact sequence of $R$-modules

$$
0 \longrightarrow \lim _{n \geq 1}^{1} t_{n} A \longrightarrow \Delta_{R, S}(A) \longrightarrow \lim _{n \geq 1} A / t_{n} A \longrightarrow 0 .
$$

In other words, the natural morphism $\beta_{R, S, A}: \Delta_{R, S}(A) \longrightarrow \Lambda_{R, S}(A)$ is surjective with the kernel isomorphic to $\lim _{n \geq 1}^{1} t_{n} A$.

Proof. This is a generalization of [23, Lemma 6.7] and [27, Sublemma 4.6] (which, in turn, is a particular case of [23, Lemma 7.5]). The reason why it holds is, essentially, because the complex $K_{R, S}^{\bullet}=\left(R \rightarrow S^{-1} R\right)$ is the inductive limit of the complexes $R \stackrel{t_{n}}{\longrightarrow} R$ over the inductive system formed by the morphisms

$$
\left(R \stackrel{t_{n-1}}{\longrightarrow} R\right) \stackrel{\left(1, s_{n}\right)}{\longrightarrow}\left(R \stackrel{t_{n}}{\longrightarrow} R\right) .
$$

To give a formal proof, consider the two-term complex

$$
\bigoplus_{n=0}^{\infty} R \longrightarrow \bigoplus_{n=1}^{\infty} R
$$

with the differential taking an eventually vanishing sequence $x_{0}, x_{1}, x_{2}, \ldots \in R$ to the eventually vanishing sequence $y_{1}, y_{2}, y_{3}, \ldots \in R$ with $y_{n}=x_{n}-s_{n} x_{n-1}, n \geq 1$. We denote this complex by $T^{\bullet}$ and place it in the cohomological degrees 0 and 1 (so $T^{0}=\bigoplus_{n=0}^{\infty} R$ and $\left.T^{1}=\bigoplus_{n=1}^{\infty} R\right)$. Furthermore, denote by $T_{n}^{\bullet}$ the subcomplex

$$
\bigoplus_{i=0}^{n-1} R \longrightarrow \bigoplus_{i=1}^{n} R
$$

in the complex $T^{\bullet}$. Then the complex $T_{n}^{\bullet}$ is homotopy equivalent to the complex $R \stackrel{t_{n}}{\longrightarrow} R$; the homotopy equivalence is provided by the morphism of complexes taking a sequence of elements $x_{0}, \ldots, x_{n-1} \in R$ to the element $x_{0} \in R$ and a sequence of elements $y_{1}, \ldots, y_{n} \in R$ to the element $-s_{n} s_{n-1} \cdots s_{2} y_{1}-s_{n} s_{n-1} \cdots s_{3} y_{2}-\cdots-y_{n} \in R$. It follows that the complex $T^{\bullet}$ is quasi-isomorphic to the complex $R \longrightarrow S^{-1} R$; the quasi-isomorphism is provided by the morphism of complexes taking an eventually vanishing sequence $\left(x_{n} \in R\right)_{n=0}^{\infty}$ to the element $x_{0} \in R$ and an eventually vanishing 
sequence $\left(y_{n} \in R\right)_{n=1}^{\infty}$ to the element $-\sum_{n=1}^{\infty} y_{n} / t_{n} \in S^{-1} R$. To be more precise, the complex $T^{\bullet}$ is quasi-isomorphic to the complex $K_{R, S}^{\bullet}[-1]$.

Now we can compute the $R$-module $\Delta_{R, S}(A)=\operatorname{Ext}_{R}^{1}\left(K_{R, S}^{\bullet}, A\right)$ as $\Delta_{R, S}(A)=$ $H_{0}\left(\operatorname{Hom}_{R}\left(T^{\bullet}, A\right)\right)$. Furthermore, the complex $\operatorname{Hom}_{R}\left(T^{\bullet}, A\right)$ is the projective limit of the complexes $\operatorname{Hom}_{R}\left(T_{n}^{\bullet}, A\right)$, which form a countable directed projective system of complexes with termwise surjective morphisms between them. Hence we have a natural short exact sequence of $R$-modules

$$
\begin{aligned}
0 \longrightarrow \lim _{n}^{1} H_{1}\left(\operatorname{Hom}_{R}\left(T_{n}^{\bullet}, A\right)\right) \longrightarrow H_{0}\left(\lim _{n} \operatorname{Hom}_{R}\left(T_{n}^{\bullet}, A\right)\right) \\
\longrightarrow \lim _{n} H_{0}\left(\operatorname{Hom}_{R}\left(T_{n}^{\bullet}, A\right)\right) \longrightarrow 0,
\end{aligned}
$$

and it remains to recall that $H_{0}\left(\operatorname{Hom}_{R}\left(T_{n}^{\bullet}, A\right)\right)=H_{0}\left(A \stackrel{t_{n}}{\rightarrow} A\right)=A / t_{n} A$ and $H_{1}\left(\operatorname{Hom}_{R}\left(T_{n}^{\bullet}, A\right)\right)=H_{1}\left(A \stackrel{t_{n}}{\rightarrow} A\right)={ }_{t_{n}} A$.

Lemma 3.3. Let $R$ be a commutative ring and $S \subset R$ be an (at most) countable multiplicative subset. Let $D_{1} \longleftarrow D_{2} \longleftarrow D_{3} \longleftarrow \cdots$ be a projective system of $R$-modules such that $D_{n}$ is an $R / t_{n} R$-module for every $n \geq 1$. Then the $R$-modules (a) $\lim _{n} D_{n}$ and (b) $\lim _{n}^{1} D_{n}$ are simply right obtainable from $R / s R$-modules, $s \in S$.

Proof. This is a generalization of [27, Sublemma 4.7]. Part (a): denote by $D_{n}^{\prime} \subset$ $D_{n}$ the image of the projection map $\lim _{m} D_{m} \longrightarrow D_{n}$. Then we have $\lim _{n} D_{n}=$ $\lim _{n} D_{n}^{\prime}$, and the maps $D_{n}^{\prime} \longrightarrow D_{n-1}^{\prime}$ are surjective. Hence the $R$-module $\lim _{n} D_{n}$ is an infinitely iterated extension, in the sense of the projective limit, of the $R$-modules $D_{1}^{\prime}$ and $\operatorname{ker}\left(D_{n}^{\prime} \rightarrow D_{n-1}^{\prime}\right), \quad n \geq 2$. These are, obviously, $R / t_{n} R$-modules. The proof of part (b) is the same as in [27, Sublemma 4.7(b)].

Lemma 3.4. Let $R$ be a commutative ring and $S \subset R$ be an (at most) countable multiplicative subset. Then all $S$-contramodule $R$-modules are simply right obtainable from $R / s R$-modules, $s \in R$.

Proof. By Lemma 3.1(b), any $S$-contramodule $R$-module $C$ has the form $C=$ $\Delta_{R, S}(A)$ for some $R$-module $A$; in fact, one has $C=\Delta_{R, S}(C)$. According to Lemma 3.2 , the $R$-module $C$ is an extension of two $R$-modules, both of which, according to Lemma 3.3(a-b), are simply right obtainable from $R / s R$-modules.

Remark 3.5. Notice that the converse assertion to Lemma 3.4 obviously holds for any multiplicative subset $S$ in a commutative ring $R$ such that $\operatorname{pd}_{R} S^{-1} R \leq 1$. In fact, all the $R$-modules right 1-obtainable from $R / s R$-modules, and even all the $R$-modules right 2-obtainable from $R / s R$-modules, $s \in S$, are $S$-contramodules. This follows simply from the fact that the class of $S$-contramodule $R$-modules is closed under kernels, cokernels, extensions, and infinite products (which implies closedness under projective limits, which can be expressed as kernels of morphisms between products) by Lemma 3.1(a), together with the fact that all $R / s R$-modules are $S$-contramodules [24, Lemma 1.6(b)]. 
3.2. Matlis multiplicative subsets of nonzero-divisors. A Matlis multiplicative subset $S$ in a commutative ring $R$ is a multiplicative subset $S \subset R$ such that $\operatorname{pd}_{R} S^{-1} R \leq 1$. All the results concerning uncountable Matlis multiplicative subsets in this paper are based on the following theorem from the paper [1].

Theorem 3.6. Let $R$ be a commutative ring and $S \subset R$ be a multiplicative subset consisting of (some) nonzero-divisors in $R$. Then the projective dimension of the $R$-module $S^{-1} R$ does not exceed 1 if and only if the quotient $R$-module $S^{-1} R / R$ is isomorphic to a direct sum of countably presented $R$-modules.

Proof. This is $[1$, Theorem $1.1(1) \Leftrightarrow(5)]$. The argument combines two techniques: Hamsher's "restrictions" [16] and tight systems. Another proof, using tilting theory rather than tight systems, can be found in $[13$, Theorem $14.59(\mathrm{a}) \Leftrightarrow(\mathrm{c})]$ (the required result from tilting theory is "deconstruction to countable type", another proof of which can be found in [30, Theorem 3.6]). For the particular case when $R$ is an integral domain, see [10, Corollary 2.8].

Let us have a little discussion of what Theorem 3.6 entails. Let $R$ be a commutative ring and $S \subset R$ be a multiplicative subset of nonzero-divisors. Then, for any multiplicative subset $T \subset S \subset R$, one has $T^{-1} R \subset S^{-1} R$ and $T^{-1} R / R \subset S^{-1} R / R$. Obviously, any countable subset in $S^{-1} R / R$ is contained in $T^{-1} R / R$ for some countable multiplicative subset $T \subset S$.

Furthermore, if an $R$-submodule $M \subset S^{-1} R / R$ is a direct summand in $S^{-1} R / R$ and $T \subset S$ is a multiplicative subset such that $M \subset T^{-1} R / R \subset S^{-1} R / R$, then $M$ is a direct summand in $T^{-1} R / R$ as well. Thus, it follows from Theorem 3.6 that, for any Matlis multiplicative subset of nonzero-divisors $S$ in a commutative ring $R$, the quotient module $S^{-1} R / R$ is a direct sum of direct summands of the quotient modules $T^{-1} R / R$, where $T$ runs over countable multiplicative subsets in $R$ contained in $S$.

Proposition 3.7. Let $R$ be a commutative ring and $S \subset R$ be a multiplicative subset of nonzero-divisors such that $\mathrm{pd}_{R} S^{-1} R \leq 1$. Then the functor $\Delta_{R, S}: R$-mod $\longrightarrow$ $R-\bmod _{S \text {-ctra }}$ is isomorphic to an infinite product of functors $\Delta_{R, S}^{\alpha}$,

$$
\Delta_{R, S}(A)=\prod_{\alpha} \Delta_{R, S}^{\alpha}(A) \quad \text { for all } A \in R \text {-mod, }
$$

indexed by some set of indices $\{\alpha\}$ and such that for every $\alpha$ there is a countable multiplicative subset $T_{\alpha} \subset S$ for which the functor $\Delta_{R, S}^{\alpha}$ is a direct summand in the functor $\Delta_{R, T_{\alpha}}$,

$$
\Delta_{R, T_{\alpha}}(A)=\Delta_{R, S}^{\alpha}(A) \oplus^{\prime} \Delta_{R, S}^{\alpha}(A) \quad \text { for all } A \in R \text {-mod. }
$$

Proof. For any multiplicative subset of nonzero-divisors $S \subset R$, the complex $K_{R, S}^{\bullet}=\left(R \rightarrow S^{-1} R\right)$ is quasi-isomorphic to the quotient module $S^{-1} R / R$, so one has $\Delta_{R, S}(A)=\operatorname{Ext}_{R}^{1}\left(S^{-1} R / R, A\right)$. Now if $\operatorname{pd}_{R} S^{-1} R \leq 1$ then, according to the preceding discussion, we have $S^{-1} R / R=\bigoplus_{\alpha} L_{\alpha}$, where the $R$-modules $L_{\alpha}$ are direct summands in the quotient modules $T_{\alpha}^{-1} R / R$ for some countable multiplicative subsets $T_{\alpha} \subset S$. It remains to set $\Delta_{R, S}^{\alpha}=\operatorname{Ext}_{R}^{1}\left(L_{\alpha},-\right)$. 
Lemma 3.8. Let $R$ be a commutative ring and $S \subset R$ be a multiplicative subset of nonzero-divisors such that $\operatorname{pd}_{R} S^{-1} R \leq 1$. Then all $S$-contramodule $R$-modules are simply right obtainable from $R / s R$-modules, $s \in S$.

Proof. Let $C$ be an $S$-contramodule $R$-module. By Lemma 3.1(b) and Proposition 3.7, we have $C=\Delta_{R, S}(C)=\prod_{\alpha} \Delta_{R, S}^{\alpha}(C)$, where the $R$-modules $\Delta_{R, S}^{\alpha}(C)$ are direct summands in the $R$-modules $\Delta_{R, T_{\alpha}}(C)$ for some countable multiplicative subsets $T_{\alpha} \subset S \subset R$. By Lemma 3.4, every $R$-module $\Delta_{R, T_{\alpha}}(C)$ is simply right obtainable from $R / t R$-modules, $t \in T_{\alpha} \subset S$, hence it follows that the $R$-module $C$ is simply right obtainable from $R / s R$-modules, $s \in S$.

3.3. Matlis multiplicative subsets with bounded torsion. Let $R$ be a commutative ring and $S \subset R$ be a multiplicative subset. Then the maximal $S$-torsion submodule $\Gamma_{S}(R) \subset R$ is the kernel ideal of the ring homomorphism $R \longrightarrow S^{-1} R$. Set $I=\Gamma_{S}(R)$ and denote by $\bar{R}$ the quotient ring $\bar{R}=R / I$.

For any multiplicative subset $T \subset R$, the image of $T$ under the ring homomorphism $R \longrightarrow \bar{R}$ is a multiplicative subset $\bar{T} \subset \bar{R}$. The localization of the $R$-module $\bar{R}$ at the multiplicative subset $T \subset R$ is the same thing as the localization of the ring $\bar{R}$ at the multiplicative subset $\bar{T} \subset \bar{R}$, that is $T^{-1} \bar{R}=\bar{T}^{-1} \bar{R}$.

In particular, we denote by $\bar{S} \subset \bar{R}$ the image of the multiplicative subset $S \subset R$ in the ring $\bar{R}$. Then we have a natural isomorphism of rings $S^{-1} R=\bar{S}^{-1} \bar{R}$ and a commutative diagram of ring homomorphisms $R \longrightarrow \bar{R} \longrightarrow S^{-1} \bar{R}=S^{-1} R$. The multiplicative subset $\bar{S} \subset \bar{R}$ is a multiplicative subset of nonzero-divisors in $\bar{R}$.

We are indebted to Silvana Bazzoni for the idea to use the simple observation that is formulated in the next lemma.

Lemma 3.9. Let $R$ be a commutative ring and $S \subset R$ be a multiplicative subset such that $\operatorname{pd}_{R} S^{-1} R \leq 1$. Then one has $\operatorname{pd}_{\bar{R}} \bar{S}^{-1} \bar{R} \leq 1$.

Proof. Clearly, $\bar{S}^{-1} \bar{R}=\bar{R} \otimes_{R} S^{-1} R$. For any ring homomorphism $R \longrightarrow R^{\prime}$ and any flat $R$-module $F$, one has $\operatorname{pd}_{R^{\prime}}\left(R^{\prime} \otimes F\right) \leq \operatorname{pd}_{R} F$, as tensoring with $R^{\prime}$ over $R$ transforms a projective resolution of the $R$-module $F$ into a projective resolution of the $R^{\prime}$-module $R^{\prime} \otimes_{R} F$.

The following two results from the paper [1], forming together a slightly more precise version of Theorem 3.6, will be needed for the purposes of this section.

Theorem 3.10. Let $R$ be a commutative ring and $S \subset R$ be a multiplicative subset of nonzero-divisors such that $\operatorname{pd}_{R} S^{-1} R \leq 1$. Then for any countable multiplicative subset $T_{0} \subset S$ there exists a countable multiplicative subset $T_{0} \subset T_{1} \subset S$ such that the $R$-submodule $T_{1}^{-1} R / R \subset S^{-1} R / R$ is a direct summand.

Proof. This is [1, Corollary 4.4]. (For the particular case when $R$ is an integral domain, see [10, sentence after Theorem 2.7].)

Theorem 3.11. Let $R$ be a commutative ring and $S \subset R$ be a multiplicative subset of nonzero-divisors such that $\operatorname{pd}_{R} S^{-1} R \leq 1$. Then every direct summand in the $R$-module $S^{-1} R / R$ is isomorphic to a direct sum of countably presented $R$-modules. 
Proof. This is [1, Theorem 7.11].

Lemma 3.12. Let $R$ be a commutative ring and $S \subset R$ be a multiplicative subset such that the $S$-torsion in $R$ is bounded and $\operatorname{pd}_{\bar{R}} \bar{S}^{-1} \bar{R} \leq 1$. Then the two-term complex of $R$-modules $K_{R . S}^{\bullet}$ is isomorphic, as an object of the derived category $\mathrm{D}(R$-mod), to a direct sum of complexes of $R$-modules $L_{\alpha}^{\bullet}$ such that, for every $\alpha$, there exists a countable multiplicative subset $T_{\alpha} \subset S \subset R$ for which the complex $L_{\alpha}^{\bullet}$, viewed as an object of $\mathrm{D}(R$-mod $)$, is a direct summand in the complex $K_{R, T_{\alpha}}$.

Proof. Let $t_{0} \in S$ be an element such that $t_{0} I=0$. By Theorem 3.10 applied to the commutative ring $\bar{R}$ with a multiplicative subset of nonzero-divisors $\bar{S} \subset \bar{R}$, there exists a countable multiplicative subset $\bar{T}_{1} \subset \bar{S}$ containing the image $\bar{t}_{0} \in \bar{R}$ of the element $t_{0} \in R$ and such that the $\bar{R}$-submodule $\bar{T}_{1}^{-1} \bar{R} / \bar{R}$ is a direct summand in the $\bar{R}$-module $\bar{S}^{-1} \bar{R} / \bar{R}$. Lifting every element of $\bar{T}_{1}$ to an element of $S \subset R$ and taking the multiplicative closure, we obtain a countable multiplicative subset $T_{1} \subset R$ such that $t_{0} \in T_{1} \subset S$ and the image of $T_{1}$ in $\bar{R}$ coincides with $\bar{T}_{1} \subset \bar{R}$.

Let $M \subset \bar{S}^{-1} \bar{R} / \bar{R}$ denote an $\bar{R}$-submodule such that $\bar{S}^{-1} \bar{R} / \bar{R}=\bar{T}_{1}^{-1} \bar{R} / \bar{R} \oplus M$. By Theorem 3.11, the $\bar{R}$-module $M$ is a direct sum of countably generated $\bar{R}$-modules $L_{\beta}$. According to the discussion in Section 3.2, for every $\beta$ there exists a countable multiplicative subset $\bar{T}_{\beta} \subset \bar{S}$ such that $L_{\beta}$ is a direct summand in $\bar{T}_{\beta}^{-1} \bar{R} / \bar{R}$. Arguing as above, we lift the multiplicative subset $\bar{T}_{\beta} \subset \bar{S} \subset \bar{R}$ to a countable multiplicative subset $T_{\beta} \subset S \subset R$. Enlarging the multiplicative subsets $\bar{T}_{\beta}$ and $T_{\beta}$ if necessary, we can assume that $\bar{T}_{1} \subset \bar{T}_{\beta}$ and $T_{1} \subset T_{\beta}$ for all $\beta$.

Now, two-term complexes of $R$-modules $K^{-1} \longrightarrow K^{0}$, viewed as objects of the derived category $\mathrm{D}(R$-mod $)$, are classified by triples $\left(H^{-1}, H^{0}, \xi\right)$, where $H^{-1}=$ $H^{-1}\left(K^{\bullet}\right)$ and $H^{0}=H^{0}\left(K^{\bullet}\right)$ are the cohomology $R$-modules, and $\xi$ is an extension class $\xi \in \operatorname{Ext}_{R}^{2}\left(H^{0}, H^{-1}\right)$. In the case of the complex $K_{R, S}^{\bullet}$, we have $H^{-1}\left(K_{R, S}^{\bullet}\right)=I$ and $H^{0}\left(K_{R, S}^{\bullet}\right)=\bar{S}^{-1} \bar{R} / \bar{R}$. For any $T_{1}^{-1} R$-module $L$, one has $\operatorname{Ext}_{R}^{*}(L, I)=0$, because the element $t_{0} \in R$ acts by an automorphism of $L$ and by zero in $I$.

In particular, $M=\bar{S}^{-1} \bar{R} / \bar{T}_{1}^{-1} \bar{R}$ is a $\bar{T}_{1}^{-1} \bar{R}$-module. So we have $\operatorname{Ext}_{R}^{2}(M, I)=0$ and $\bar{S}^{-1} \bar{R} / \bar{R}=\bar{T}_{1}^{-1} \bar{R} / \bar{R} \oplus M$, hence it follows that the complex $K_{R, S}^{\bullet}$ is isomorphic to the direct sum of its subcomplex $K_{R, T_{1}}^{\bullet}$ and the $R$-module $M$ in $\mathrm{D}(R$-mod).

Similarly, in the case of the complex $K_{R, T_{\beta}}^{\bullet}$, we have $H^{-1}\left(K_{R, T_{\beta}}^{\bullet}\right)=I$ and $H^{0}\left(K_{R, T_{\beta}}^{\bullet}\right)=\bar{T}_{\beta}^{-1} \bar{R} / \bar{R}$. Since $\operatorname{Ext}_{R}^{2}\left(L_{\beta}, I\right)=0$ and the $\bar{R}$-module $L_{\beta}$ is a direct summand in the $\bar{R}$-module $\bar{T}_{\beta}^{-1} \bar{R} / \bar{R}$, it follows that the $R$-module $L_{\beta}$ is a direct summand of the complex $K_{R, T_{\beta}}^{\bullet}$ in $\mathrm{D}(R$-mod).

We have shown that $K_{R, S}^{\bullet} \simeq K_{R, T_{1}}^{\bullet} \oplus \bigoplus_{\beta} L_{\beta}$ in $\mathrm{D}(R$-mod $)$, where $L_{\beta}$ is a direct summand of $K_{R, T_{\beta}}$ in $\mathrm{D}(R$-mod $)$. It remains to define the set of indices $\{\alpha\}$ to be the disjoint union of the set of indices $\{\beta\}$ and the one-element set $\{1\}$, that is $\{\alpha\}=\{\beta\} \sqcup\{1\}$. Put $L_{\beta}^{\bullet}=L_{\beta}$ and $L_{1}^{\bullet}=K_{R, T_{1}}^{\bullet}$.

The following corollary provides a partial converse assertion to Lemma 3.9. 
Corollary 3.13. Let $R$ be a commutative ring and $S \subset R$ be a multiplicative subset such that the $S$-torsion in $R$ is bounded and $\operatorname{pd}_{\bar{R}} \bar{S}^{-1} \bar{R} \leq 1$. Then $\operatorname{pd}_{R} S^{-1} R \leq 1$.

Proof. For any bounded complex of $R$-modules $K^{\bullet}$, denote by $\operatorname{pd}_{R} K^{\bullet}$ the supremum of all the integers $n$ for which there exists an $R$-module $A$ such that $\operatorname{Ext}_{R}^{n}\left(K^{\bullet}, A\right) \neq 0$. So $\operatorname{pd}_{R} K^{\bullet} \in \mathbb{Z} \cup\{+\infty\}$ if $K^{\bullet} \neq 0$ in $\mathrm{D}(R-$ mod $)$, and $\operatorname{pd}_{R} K^{\bullet}=-\infty$ when the complex $K^{\bullet}$ is acyclic. For any distinguished triangle $K^{\bullet} \longrightarrow L^{\bullet} \longrightarrow M^{\bullet} \longrightarrow K^{\bullet}[1]$ in the bounded derived category $\mathrm{D}^{\mathrm{b}}(R-\bmod )$, we have $\operatorname{pd}_{R} L^{\bullet} \leq \max \left(\operatorname{pd}_{R} K^{\bullet}, \operatorname{pd}_{R} M^{\bullet}\right)$.

In particular, for any commutative ring $R$ with a multiplicative subset $S \subset R$ we have $\operatorname{pd}_{R} K_{R, S}^{\bullet} \leq 1$ if and only if $\operatorname{pd}_{R} S^{-1} R \leq 1$, because $\operatorname{pd}_{R} R=0$. Now if the $S$-torsion in $R$ is bounded and $\operatorname{pd}_{\bar{R}} \bar{S}^{-1} \bar{R} \leq 1$, then we can apply Lemma 3.12, obtaining a direct sum decomposition $K_{R, S}^{\bullet} \simeq \bigoplus_{\alpha} L_{\alpha}^{\bullet}$ in $\mathrm{D}^{\mathrm{b}}\left(R\right.$-mod), where $L_{\alpha}^{\bullet}$ is a direct summand of $K_{R, T_{\alpha}}^{\bullet}$ in $\mathrm{D}^{\mathrm{b}}\left(R\right.$-mod) and the multiplicative subset $T_{\alpha} \subset S \subset R$ is countable. By [24, Lemma 1.9], we have $\operatorname{pd}_{R} T_{\alpha}^{-1} R \leq 1$, hence $\operatorname{pd}_{R} K_{R, T_{\alpha}}^{\bullet} \leq 1$ and $\operatorname{pd}_{R} L_{\alpha}^{\bullet} \leq 1$ for all $\alpha$. Thus $\operatorname{pd}_{R} K_{R, S}^{\bullet} \leq 1$, and it follows that $\operatorname{pd}_{R} S^{-1} R \leq 1$.

The next proposition is a generalization of Proposition 3.7 to the case of bounded torsion.

Proposition 3.14. Let $R$ be a commutative ring and $S \subset R$ be a multiplicative subset such that the $S$-torsion in $R$ is bounded and $\operatorname{pd}_{R} S^{-1} R \leq 1$. Then the functor $\Delta_{R, S}: R-\bmod \longrightarrow R-\bmod _{S \text {-ctra }}$ is isomorphic to an infinite product of functors $\Delta_{R, S}^{\alpha}$,

$$
\Delta_{R, S}(A)=\prod_{\alpha} \Delta_{R, S}^{\alpha}(A) \quad \text { for all } A \in R-\bmod
$$

indexed by some set of indices $\{\alpha\}$ and such that for every $\alpha$ there is a countable multiplicative subset $T_{\alpha} \subset S$ for which the functor $\Delta_{R, S}^{\alpha}$ is a direct summand in the functor $\Delta_{R, T_{\alpha}}$,

$$
\Delta_{R, T_{\alpha}}(A)=\Delta_{R, S}^{\alpha}(A) \oplus^{\prime} \Delta_{R, S}^{\alpha}(A) \quad \text { for all } A \in R \text {-mod. }
$$

Proof. In view of Lemma 3.9, Lemma 3.12 is applicable, and it remains to recall that $\Delta_{R, S}=\operatorname{Ext}_{R}^{1}\left(K_{R, S}^{\bullet},-\right)$ and $\Delta_{R, T_{\alpha}}=\operatorname{Ext}_{R}^{1}\left(K_{R, T_{\alpha}}^{\bullet},-\right)$.

Lemma 3.15. Let $R$ be a commutative ring and $S \subset R$ be a multiplicative subset such that the $S$-torsion in $R$ is bounded and $\operatorname{pd}_{R} S^{-1} R \leq 1$. Then all $S$-contramodule $R$-modules are simply right obtainable from $R / s R$-modules, $s \in S$.

Proof. Similar to the proof of Lemma 3.8, using Lemma 3.1(b), Proposition 3.14, and Lemma 3.4.

\section{Rings with a Multiplicative Subset}

We start with a proof of Proposition 1.2 and then proceed to prove Propositions 1.6-1.8 and Theorems 1.3-1.5 (which is the aim of this section).

Let $R$ be a commutative ring and $S \subset R$ be a multiplicative subset. Recall that an $R$-module $C$ is said to be $S$-weakly cotorsion if $\operatorname{Ext}_{R}^{1}\left(S^{-1} R, C\right)=0$, and an 
$R$-module $F$ is said to be $S$-strongly flat if $\operatorname{Ext}_{R}^{1}(F, C)=0$ for all $S$-weakly cotorsion $R$-modules $C$.

An $R$-module $M$ is said to be $S$-divisible if $s M=M$ for all $s \in S$. An $R$-module $M$ is said to be $S$-h-divisible if there exists a surjective $R$-module morphism onto $M$ from an $S^{-1} R$-module. Any $S$-h-divisible $R$-module is $S$-divisible. An $R$-module $M$ is $S$-h-divisible if and only if the natural morphism $\operatorname{Hom}_{R}\left(S^{-1} R, M\right) \longrightarrow M$ is surjective. More precisely, for any $R$-module $M$ let us denote the image of the morphism $\operatorname{Hom}_{R}\left(S^{-1} R, M\right) \longrightarrow M$ by $h_{S}(M) \subset M$. Then $h_{S}(M)$ is the (unique) maximal $S$-h-divisible submodule of $M$. An $R$-module $M$ is said to be $S$-h-reduced if it has no $S$-h-divisible submodules, that is $h_{S}(M)=0$. An $R$-module $M$ is $S$-hreduced if and only if $\operatorname{Hom}_{R}\left(S^{-1} R, M\right)=0$. We refer to [24, Section 1] and the references therein for further discussion.

Proof of Proposition 1.2. For any commutative $\operatorname{ring} R$ with a multiplicative subset $S \subset R$, the class of all flat $R$-modules $F$ such that the $R / s R$-module $F / s F$ is projective for all $s \in S$ and the $S^{-1} R$-module $S^{-1} F$ is projective is closed under kernels of surjective morphisms (as well as under extensions and infinite direct sums, and more generally, transfinitely iterated extensions in the sense of the inductive limit). Since the $R$-module $S^{-1} R$ belongs to this class, so does its first syzygy $R$-module $H$.

On the other hand, assume that the multiplicative subset $S \subset R$ consists of (some) nonzero-divisors in a $R$. Suppose that the $R$-module $H$ is $S$-strongly flat. Then we have $\operatorname{Ext}_{R}^{2}\left(S^{-1} R, C\right)=\operatorname{Ext}_{R}^{1}(H, C)=0$ for all $S$-weakly cotorsion $R$-modules $C$. Hence the cokernel of any injective morphism of $S$-weakly cotorsion $R$-modules is an $S$-weakly cotorsion $R$-module.

For the purposes of this proof, let $K$ denote the quotient $R$-module $S^{-1} R / R$. Following the argument in [13, proof of Lemma $7.53(\mathrm{c}) \Rightarrow(\mathrm{a})]$ and using $[24$, Lemma 1.7(a)], one shows that the $R$-module $\operatorname{Ext}_{R}^{1}(K, M)$ is $S$-h-reduced for any $R$-module $M$, hence the $R$-module $M / h_{S}(M)$ is $S$-h-reduced for any $M$, and therefore the class of all $S$-h-divisible $R$-modules is closed under extensions. Finally, it remains to apply [24, Lemma $1.8(\mathrm{~b})]$ in order to conclude that the projective dimension of the $R$-module $S^{-1} R$ does not exceed 1 .

As in Section 3, we denote by $K_{R, S}^{\bullet}$ the two-term complex of $R$-modules $R \longrightarrow$ $S^{-1} R$, with the term $R$ sitting in the cohomological degree -1 and the term $S^{-1} R$ in the cohomological degree 0 . We also denote simply by $S^{-1} R / R$ the cokernel of the $R$-module morphism $R \longrightarrow S^{-1} R$. The functor $A \longmapsto \operatorname{Ext}_{R}^{1}\left(K_{R, S}^{\bullet}, A\right)=$ $\operatorname{Hom}_{\mathrm{D}(R-\bmod )}\left(K_{R, S}^{\bullet}, A[1]\right)$ is denoted by $\Delta_{R, S}: R-\bmod \longrightarrow R-\bmod$.

Lemma 4.1. Let $R$ be commutative ring and $S \subset R$ be a multiplicative subset. Then for any $R$-module $A$ there is a natural 5-term exact sequence of $R$-modules

$$
\begin{aligned}
0 \longrightarrow \operatorname{Hom}_{R}\left(S^{-1} R / R, A\right) & \longrightarrow \operatorname{Hom}_{R}\left(S^{-1} R, A\right) \\
& \longrightarrow A \longrightarrow \Delta_{R, S}(A) \longrightarrow \operatorname{Ext}_{R}^{1}\left(S^{-1} R, A\right) \longrightarrow 0
\end{aligned}
$$


which in the case of an $S$-weakly cotorsion $R$-module $C$ reduces to a 4-term exact sequence

(3)

$0 \longrightarrow \operatorname{Hom}_{R}\left(S^{-1} R / R, C\right) \longrightarrow \operatorname{Hom}_{R}\left(S^{-1} R, C\right) \longrightarrow C \longrightarrow \Delta_{R, S}(C) \longrightarrow 0$.

Proof. Apply the cohomological functor $\operatorname{Hom}_{\mathrm{D}(R-\bmod )}(-, A[*])$ to the distinguished triangle

$$
R \longrightarrow S^{-1} R \longrightarrow K_{R, S}^{\bullet} \longrightarrow R[1]
$$

in the derived category $\mathrm{D}(R-$ mod $)$.

The next lemma uses the definitions from Section 3.

Lemma 4.2. Let $R$ be a commutative ring and $S \subset R$ be a multiplicative subset such that $\operatorname{pd}_{R} S^{-1} R \leq 1$. Then for any $S$-torsion $R$-module $D$ and any $R$-module $A$ the $R$-module $\operatorname{Hom}_{R}(D, A)$ is an $S$-contramodule.

Proof. Recall the notation ${ }_{r} D \subset D$ for the kernel of the multiplication map $r: D \longrightarrow$ $D, r \in R$. Then one has $D=\lim _{s \in S} s$, hence $\operatorname{Hom}_{R}(D, A)=\lim _{s \in S} \operatorname{Hom}_{R}\left({ }_{s} D, A\right)$. Now the $R$-module $\operatorname{Hom}_{R}\left({ }_{s} D, A\right)$ is annihilated by the action of the element $s \in S$, so it is an $S$-contramodule by [24, Lemma 1.6(b)]. By Lemma 3.1(a), the class of $S$-contramodule $R$-modules is closed under infinite products and kernels of morphisms in $R$-mod, hence it is also closed under all projective limits.

In fact, the assumption of projective dimension of $S^{-1} R$ not exceeding 1 is not needed for the validity of this lemma; see [20, Theorem 2.1] and [24, Lemma 1.4 or proof of Lemma 1.7(a)] (but we have only defined $S$-contramodule $R$-modules in the assumption of $\left.\operatorname{pd}_{R} S^{-1} R \leq 1\right)$.

Lemma 4.3. Let $R \longrightarrow R^{\prime}$ be a homomorphism of commutative rings and $F$ be a flat $R$-module. Then for any $R^{\prime}$-module $C^{\prime}$ and all $i \geq 0$ there is a natural isomorphism of Ext groups/modules $\operatorname{Ext}_{R}^{i}\left(F, C^{\prime}\right) \simeq \operatorname{Ext}_{R^{\prime}}^{i}\left(R^{\prime} \otimes_{R} F, C^{\prime}\right)$.

Proof. This is a particular case of [27, Lemma 4.1(a)].

Proof of Propositions 1.6-1.8. In all the cases covered by the assumptions of these three propositions, one has $\operatorname{pd}_{R} S^{-1} R \leq 1$, so $\Delta_{R, S}(A)$ is an $S$-contramodule $R$-module for any $R$-module $A$ (see Lemma 3.1(b) and/or [24, Lemma 1.7(c)]).

Now let $C$ be an $S$-weakly cotorsion $R$-module. We will prove a stronger assertion, viz., that $C$ is simply right obtainable from $R / s R$-modules, $s \in S$, and an $S^{-1} R$-module (see Section 2 for the definitions).

The exact sequence (3) represents $C$ as an extension of an $S$-contramodule $\Delta_{R, S}(C)$ and the cokernel of an injective morphism from an $S$-contramodule $\operatorname{Hom}_{R}\left(S^{-1} R / R, C\right.$ ) (see Lemma 4.2) into an $S^{-1} R$-module $\operatorname{Hom}_{R}\left(S^{-1} R, C\right.$ ). So any $S$-weakly cotorsion $R$-module is simply right obtainable from two $S$-contramodule $R$-modules and one $S^{-1} R$-module.

It remains to recall that, as we already know, in the assumptions of any of the three propositions all $S$-contramodule $R$-modules are simply right obtainable from $R / s R$-modules, $s \in S$. In the case of Proposition 1.6, this is the result of Lemma 3.4; 
in the case of Proposition 1.7, we have Lemma 3.8; and in the case of Proposition 1.8, we need to apply Lemma 3.15.

This proves the "only if" assertions of the three propositions. The "if" assertion holds for any multiplicative subset $S$ in a commutative ring $R$. To prove as much, denote by $\mathrm{E} \subset R$-mod the class of all $R / s R$-modules, $s \in S$, and all $S^{-1} R$-modules (viewed as $R$-modules via the restriction of scalars).

By Lemma 4.3, for any $R$-module $E \in \mathrm{E}$ we have $\operatorname{Ext}_{R}^{i}\left(S^{-1} R, E\right)=0$ for all $i>0$ (since $S^{-1} R$ is a flat $R$-module, $R / s R \otimes_{R} S^{-1} R=0$, and $S^{-1} R \otimes_{R} S^{-1} R=S^{-1} R$ is a free $S^{-1} R$-module). It remains to apply Lemma 2.4 in order to conclude that $\operatorname{Ext}_{R}^{1}\left(S^{-1} R, C\right)=0$ for all $R$-modules $C$ right 1-obtainable from $\mathrm{E}$.

Proof of Theorems 1.3-1.5. The "only if" assertion holds for any multiplicative subset $S$ in a commutative ring $R$ and follows immediately from the description of $S$-strongly flat $R$-modules in terms of the exact sequence (1).

To prove the "if", we, as above, denote by $\mathrm{E} \subset R$-mod the class of all $R / s R$-modules, $s \in S$, and all $S^{-1} R$-modules (viewed as $R$-modules via the restriction of scalars). Let $F$ be a flat $R$-module such that the $R / s R$-module $F / s F$ is projective for all $s \in S$ and the $S^{-1} R$-module $S^{-1} F$ is projective. Then, by Lemma 4.3, we have $\operatorname{Ext}_{R}^{i}(F, E)=0$ for all $E \in \mathrm{E}$ and all $i>0$.

Applying Lemma 2.4, we learn that $\operatorname{Ext}_{R}^{i}(F, C)=0$ for all $R$-modules $C$ right 1-obtainable from $\mathrm{E}$ and all $i>0$. Depending on which of the three Theorems 1.3, 1.4 , or 1.5 we want to prove, we can use one of the respective Propositions 1.6, 1.7, or 1.8, which tells that all $S$-weakly cotorsion $R$-modules $C$ are right 1 -obtainable from E. Thus $\operatorname{Ext}_{R}^{1}(F, C)=0$ for all $S$-weakly cotorsion $R$-modules $C$, that is, $F$ is an $S$-strongly flat $R$-module.

\section{Rings with Several Multiplicative Subsets}

The aim of this section is to prove Proposition 1.11 and Theorem 1.10. The arguments here generalize those in [27, Section 8].

Lemma 5.1. Let $f: R \longrightarrow R^{\prime}$ be a homomorphism of commutative rings, $S \subset R$ be a multiplicative subset, and $S^{\prime}=f(S) \subset R$ be the image of $S$ in $R^{\prime}$. In this setting:

(a) if $\operatorname{pd}_{R} S^{-1} R \leq 1$, then $\operatorname{pd}_{R^{\prime}} S^{\prime-1} R^{\prime} \leq 1$;

(b) an $R^{\prime}$-module is an $S$-weakly cotorsion $R$-module if and only if it is an $S^{\prime}$-weakly cotorsion $R^{\prime}$-module;

(c) an $R^{\prime}$-module is an $S$-contramodule $R$-module if and only if it is an $S^{\prime}$-contramodule $R^{\prime}$-module.

Proof. Part (a) was already explained in the proof of Lemma 3.9, while parts (b) and (c) follow from Lemma 4.3.

Lemma 5.2. Let $R$ be a commutative ring, $s \in R$ be an element, and $S \subset R$ be a multiplicative subset such that $\operatorname{pd}_{R} S^{-1} R \leq 1$. Let $K^{\bullet}$ be a complex of $R$-modules and $C$ be an $R$-module. Then 
(a) the $R$-module $\operatorname{Hom}_{R}\left(K^{\bullet}, C[i]\right)$ is annihilated by $s$ for all $i \in \mathbb{Z}$ whenever the $R$-module $C$ is annihilated by $s$;

(b) the $R$-module $\operatorname{Hom}_{R}\left(K^{\bullet}, C[i]\right)$ is an $S$-contramodule for all $i \in \mathbb{Z}$ whenever the $R$-module $C$ is an $S$-contramodule.

Proof. Part (a) holds because, for any $R$-module $C$, the multiplication map $s: \operatorname{Hom}_{R}\left(K^{\bullet}, C\right) \longrightarrow \operatorname{Hom}_{R}\left(K^{\bullet}, C\right)$ is induced by the multiplication map $s: C \longrightarrow$ $C$. Part (b) is a (partial) generalization of the related assertion of [23, Lemma 6.2(b)]. Since $\operatorname{pd}_{R} S^{-1} R \leq 1$, for any complex of $R$-modules $B^{\bullet}$ there are short exact sequences

$$
\begin{aligned}
0 \longrightarrow \operatorname{Ext}_{R}^{1}\left(S^{-1} R, H^{i-1}\left(B^{\bullet}\right)\right) \longrightarrow \\
H^{i}\left(\mathbb{R} \operatorname{Hom}_{R}\left(S^{-1} R, B^{\bullet}\right)\right) \longrightarrow \operatorname{Hom}_{R}\left(S^{-1} R, H^{i}\left(B^{\bullet}\right)\right) \longrightarrow 0
\end{aligned}
$$

where $\mathbb{R} \operatorname{Hom}_{R}$ denotes the derived functor of $R$-module homomorphisms, viewed as a functor acting on the derived category of $R$-modules. Therefore, the $R$-modules $H^{i}\left(B^{\bullet}\right)$ are $S$-contramodules for all $i \in \mathbb{Z}$ if and only if $\mathbb{R} \operatorname{Hom}_{R}\left(S^{-1} R, B^{\bullet}\right)=0$ in $\mathrm{D}(R$-mod) (cf. [24, Lemma 4.5(b)]).

Now one has

$$
\mathbb{R} \operatorname{Hom}_{R}\left(S^{-1} R, \mathbb{R} \operatorname{Hom}_{R}\left(K^{\bullet}, C\right)\right)=\mathbb{R} \operatorname{Hom}_{R}\left(K^{\bullet}, \mathbb{R} \operatorname{Hom}_{R}\left(S^{-1} R, C\right)\right),
$$

hence acyclicity of the the complex $\mathbb{R} \operatorname{Hom}_{R}\left(S^{-1} R, C\right)$ implies acyclicity of the complex $\mathbb{R} \operatorname{Hom}_{R}\left(S^{-1} R, \mathbb{R} \operatorname{Hom}_{R}\left(K^{\bullet}, C\right)\right)$.

Let $R$ be a commutative ring and $\mathbf{S}=\left\{S_{1}, \ldots, S_{m}\right\}$ be a finite collection of multiplicative subsets in $R$. Assume that $\operatorname{pd}_{R} S_{j}^{-1} R \leq 1$ for all $j=1, \ldots, m$. We will say that an $R$-module $C$ is an $\mathbf{S}$-contramodule if it is an $S_{j}$-contramodule for every $1 \leq j \leq m$.

Let $s_{1} \in S_{1}, \ldots, s_{m} \in S_{m}$ be a sequence of elements, which will denote for brevity by a single letter $\mathbf{s}$. Then we denote by $R_{\mathbf{s}}$ the quotient ring $R_{\mathbf{s}}=R /\left(s_{1} R+\cdots+s_{m} R\right)$ of the ring $R$ by the ideal generated by the elements $s_{1}, \ldots, s_{m}$.

Lemma 5.3. Let $R$ be a commutative ring and $\mathbf{S}=\left\{S_{1}, \ldots, S_{m}\right\}$ be a finite collection of multiplicative subsets in $R$ such that, for every $1 \leq j \leq m$, either $S_{j}$ is countable, or the $S_{j}$-torsion in $R$ is bounded and $\operatorname{pd}_{R} S_{j}^{-1} R \leq 1$. Then an $R$-module $C$ is an $\mathbf{S}$-contramodule if and only if it is simply right obtainable from $R_{\mathbf{s}}$-modules, where $\mathbf{s}$ runs over all the sequences of elements $s_{1} \in S_{1}, \ldots, s_{m} \in S_{m}$.

Proof. The "if" assertion holds for any collection of Matlis multiplicative subsets $S_{j} \subset R$. In fact, any $R$-module right 1-obtainable from $R_{\mathrm{s}}$-modules is an $S_{j}$-contramodule for every $j$ by Remark 3.5, hence it is an $\mathbf{S}$-contramodule. The "only if" assertion is the nontrivial part of the lemma.

The following proof is a generalization of the "alternative" proof of [27, Lemma 8.2]. Arguing by induction on $n=0, \ldots, m$ for a fixed ring $R$ with $m$ multiplicative subsets $S_{1}, \ldots, S_{m} \subset R$, we will prove the following assertion: any $R$-module which is an $S_{j}$-contramodule for all $1 \leq j \leq n$ and which is annihilated by the ideal $s_{n+1} R+\cdots+s_{m} R \subset R$ for some elements $s_{k} \in S_{k}, \quad n+1 \leq k \leq m$, is simply right 
obtainable from $R_{\mathrm{s}}$-modules. For $n=0$, this is a trivial assertion, which provides the induction base. For $n=m$, this is the assertion of the lemma.

We do not want to pass to quotient rings $R^{\prime}=R /\left(s_{n+1} R+\cdots+s_{m} R\right)$ for the purposes of the current induction procedure because, if the $S_{j}$-torsion in $R$ is bounded and $\operatorname{pd}_{R} S_{j}^{-1} R \leq 1$ for some $1 \leq j \leq n$, and $S_{j}^{\prime}$ is the image of $S_{j}$ in $R^{\prime}$, it does not follow that the $S_{j}^{\prime}$-torsion in $R^{\prime}$ is bounded. So we will not really use Lemma 5.1 in this proof, but will rather use Lemma 5.2(a) instead.

Let $C$ be an $R$-module that is an $S_{j}$-contramodule for all $1 \leq j \leq n$, and let $s_{k} \in S_{k}$, $n+1 \leq k \leq m$, be some elements such that $s_{k} C=0$ for all $n+1 \leq k \leq m$. We have two cases, depending on whether the multiplicative subset $S_{n} \subset R$ is countable, or it is a Matlis multiplicative subset with bounded torsion.

If $S_{n}$ is countable, we follow the proof of Lemma 3.4 for $S=S_{n}$ in order to observe that $C$ is simply right obtainable from $R / s R$-modules, $s \in S_{n}$, each of which, in turn, can be obtained from $C$ in a functorial way using the operations of the passages to the kernels and cokernels of (natural) $R$-module morphisms and infinite products of $R$-modules. All such $R / s R$-modules, therefore, are $S_{j}$-contramodule $R$-modules for all $1 \leq j \leq n-1$ and are annihilated by $s_{k}$ for $n+1 \leq k \leq m$. It remains to set $s_{n}=s$ and use the induction assumption.

If the $S_{n}$-torsion in $R$ is bounded and $\operatorname{pd}_{R} S_{n}^{-1} R \leq 1$, we apply Lemma 3.1(b) and Proposition 3.14, obtaining a direct product decomposition

$$
C=\Delta_{R, S_{n}}(C)=\prod_{\alpha} \Delta_{R, S}^{\alpha}(C),
$$

where $\Delta_{R, S}^{\alpha}(C)$ are direct summands in the $R$-modules $\Delta_{R, T_{\alpha}}(C)$ for some countable multiplicative subsets $T_{\alpha} \subset S_{n}$. By Lemma 5.2(a-b), the $R$-modules $\Delta_{R, T_{\alpha}}(C)$ are annihilated by $s_{k}$ for all $n+1 \leq k \leq m$ and are $S_{j}$-contramodules for all $1 \leq j \leq n-1$.

Applying the proof of Lemma 3.4 for $S=T_{\alpha}$ to the $T_{\alpha}$-contramodules $\Delta_{R, T_{\alpha}}(C)$ and arguing as above, we see that the $R$-modules $\Delta_{R, T_{\alpha}}(C)$ are simply right obtainable from $R$-modules that are $S_{j}$-contramodules for $1 \leq j \leq n-1$, annihilated by the elements $s_{k}$ for $n+1 \leq k \leq m$, and also annihilated by some elements $s \in T_{\alpha} \subset S_{n}$. Once again, it remains to set $s_{n}=s$ and use the induction assumption.

Remark 5.4. Notice that no products of multiplicative subsets $S_{j} \subset R, j=1, \ldots, m$ are involved in the formulation of Lemma 5.3, though they are necessary in Proposition 1.11 and Theorem 1.10. The following observations may help the reader feel more comfortable: if $T \subset S \subset R$ are two embedded multiplicative subsets, then any $T$-contramodule $R$-module is an $S$-contramodule $R$-module. Indeed, $S^{-1} R$ is a $T^{-1} R$-module, so [24, Lemma 1.2] applies. On the other hand, a $T$-weakly cotorsion $R$-module does not need to be $S$-weakly cotorsion (consider the case of a trivial multiplicative subset $T=\{1\})$. Cf. [23, Remark 5.2].

Furthermore, for a pair of multiplicative subsets $S$ and $T$ in a commutative ring $R$, an $S$-weakly cotorsion and $T$-weakly cotorsion $R$-module does not have to be $S T$-weakly cotorsion. Indeed, otherwise the $R$-module $(S T)^{-1} R$ would be a direct summand in a transfinitely iterated extension of copies of the three $R$-modules $R$, $S^{-1} R$, and $T^{-1} R$. However, for any such transfinitely iterated extension $F$ and a 
nonzero element $x \in F$ there exists an $R$-submodule $G \subset F, x \in G$ and an $R$-module morphism from $G$ into either $R$, or $S^{-1} R$, or $T^{-1} R$ taking $x$ to a nonzero element. In addition, the quotient module $F / G$ is also a transfinitely iterated extension of the three $R$-modules $R, S^{-1} R$, and $T^{-1} R$. For example, consider the case of the ring of integers $R=\mathbb{Z}$ with the multiplicative subsets $S=\left\{p^{n} \mid n \in \mathbb{Z}_{\geq 0}\right\}$ and $T=\left\{q^{n} \mid n \in \mathbb{Z}_{>0}\right\}$, where $p$ and $q$ are two distinct prime numbers. Then no transfinitely iterated extension of the three $\mathbb{Z}$-modules $\mathbb{Z}, \mathbb{Z}\left[p^{-1}\right]$, and $\mathbb{Z}\left[q^{-1}\right]$ contains elements infinitely divisible by $p q$.

Lemma 5.5. Let $R$ be a commutative ring and $S, T \subset R$ be two multiplicative subsets. Denote by $S^{\prime} \subset T^{-1} R$ the image of $S$ under the localization morphism $R \longrightarrow T^{-1} R$. Assume that the $S$-torsion in $R$ is bounded. Then the $S^{\prime}$-torsion in $T^{-1} R$ is bounded.

Proof. Let $s_{0} \in S$ be an element such that $s_{0} \Gamma_{S}(R)=0$. Denote by $s_{0}^{\prime} \in S^{\prime}$ the image of the element $s_{0} \in R$ under the ring homomorphism $R \longrightarrow T^{-1} R$. Then $s_{0}^{\prime} \Gamma_{S^{\prime}}\left(T^{-1} R\right)=0$. Indeed, if $s^{\prime} r / t=0$ in $T^{-1} R$ for some $s^{\prime} \in S^{\prime}, r \in R$, and $t \in T$, then, denoting by $s \in S$ a preimage of the element $s^{\prime}$, there exists an element $u \in T$ such that $s r u=0$ in $R$. It follows that $s_{0} r u=0$ in $R$, hence $s_{0}^{\prime} r / t=0$ in $T^{-1} R$.

Lemma 5.6. Let $R$ be a commutative ring and $S, T \subset R$ be two multiplicative subsets. Assume that an $R$-module $C$ is $S T$-weakly cotorsion. Then the $R$-module $\operatorname{Hom}_{R}\left(T^{-1} R, C\right)$ is $S$-weakly cotorsion.

Proof. According to [27, proof of Lemma 8.6], for any flat $R$-modules $F$ and $G$ the $R$-module $\operatorname{Ext}_{R}^{1}\left(F, \operatorname{Hom}_{R}(G, C)\right)$ is a submodule in the $R$-module $\operatorname{Ext}_{R}^{1}\left(F \otimes_{R} G, C\right)$. In particular, the $R$-module $\operatorname{Ext}_{R}^{1}\left(S^{-1} R, \operatorname{Hom}_{R}\left(T^{-1} R, C\right)\right)$ is a submodule in the $R$-module $\operatorname{Ext}_{R}^{1}\left((S T)^{-1} R, C\right)$, so the former vanishes whenever the latter does.

Now we return to the notation of Section 1.4. Suppose that we have a commutative ring $R$ with several multiplicative subsets $S_{1}, \ldots, S_{m} \subset R$. For any subset of indices $J \subset\{1, \ldots, m\}$ we denote by $S_{J}$ the multiplicative subset $\prod_{j \in J} S_{j} \subset R$. The collection of $m$ multiplicative subsets $\left\{S_{j} \mid 1 \leq j \leq m\right\}$ is denoted by $\mathbf{S}$, and the collection of $2^{m}$ multiplicative subsets $\left\{S_{J} \mid J \subset\{1, \ldots, m\}\right\}$ is denoted by $\mathbf{S}^{\times}$.

Given a subset of indices $J \subset\{1, \ldots, m\}$, we denote its complement by $K=$ $\{1, \ldots, m\} \backslash J$. The symbol $\mathbf{s}$ denotes an arbitrary collection of elements $s_{k} \in S_{k}$, $k \in K$, and the ring $R_{J, \mathbf{s}}$ is defined as the quotient ring of $S_{J}^{-1} R$ by the ideal generated by the elements $s_{k}, k \in K$.

Proof of Proposition 1.11. This proof is a generalization of the proof of [27, Main Proposition 8.1], and the reader may wish to glance into the discussion in [27] for motivation related to the inductive argument below.

We will prove the following assertion by induction on a pair of integers $0 \leq$ $n \leq m$ : any $R$-module that is $S_{J}$-weakly cotorsion for all $J \subset\{1, \ldots, n\}$ and an $S_{k}$-contramodule for all $n+1 \leq k \leq m$ is right 1-obtainable from $R_{J, \mathbf{s}}$-modules, where $J \subset\{1, \ldots, n\} \subset\{1, \ldots, m\}$ and $\mathbf{s}$ runs over all the collections of elements 
$s_{k} \in S_{k}, \quad k \in K=\{1, \ldots, m\} \backslash J$. For $n=m$, this is the "only if" assertion of the proposition.

Induction base: for $n=0$, this is the assertion of Lemma 5.3.

Let $C$ be an $R$-module that is $S_{J}$-weakly cotorsion for all $J \subset\{1, \ldots, n\}$ and an $S_{k}$-contramodule for all $n+1 \leq k \leq m$. Consider the exact sequence (3) (from Lemma 4.1) for the multiplicative subset $S=S_{n}$ :

(4)

$0 \longrightarrow \operatorname{Hom}_{R}\left(S_{n}^{-1} R / R, C\right) \longrightarrow \operatorname{Hom}_{R}\left(S_{n}^{-1} R, C\right) \longrightarrow C \longrightarrow \Delta_{R, S_{n}}(C) \longrightarrow 0$.

Denote by $\mathrm{E}_{n} \subset R$-mod the class of all $R_{J, \mathrm{~s}}$-modules with $J \subset\{1, \ldots, n\} \subset$ $\{1, \ldots, m\}$. To show that $C$ is right 1 -obtainable from $\mathrm{E}_{n}$, it suffices to check that the $R$-modules $\operatorname{Hom}_{R}\left(S_{n}^{-1} R, C\right)$ and $\Delta_{R, S_{n}}(C)$ are right 1-obtainable from $\mathrm{E}_{n}$ and the $R$-module $\operatorname{Hom}_{R}\left(S_{n}^{-1} R / R, C\right)$ is right 2-obtainable from $\mathrm{E}_{n}$. Let us consider these three $R$-modules one by one.

The $R$-module $\operatorname{Hom}_{R}\left(S_{n}^{-1} R, C\right)$ is an $S_{n}^{-1} R$-module. By Lemma $5.2(\mathrm{~b})$, it is also an $S_{k}$-contramodule for all $n+1 \leq k \leq m$, and by Lemma 5.6 , it is $S_{J}$-weakly cotorsion for all $J \subset\{1, \ldots, n-1\}$. In order to conclude that the $R$-module $\operatorname{Hom}_{R}\left(S_{n}^{-1} R, C\right)$ is right 1-obtainable from $\mathrm{E}_{n}$, we apply the induction assumption to the pair of integers $\left(n^{\prime}, m^{\prime}\right)=(n-1, m-1)$, the ring $R^{\prime}=S_{n}^{-1} R$, and the collection of $m-1$ multiplicative subsets $S_{1}^{\prime}, \ldots, S_{n-1}^{\prime}, S_{n+1}^{\prime}, \ldots, S_{m}^{\prime} \subset R^{\prime}$ obtained as the images of the multiplicative subsets $S_{1}, \ldots, S_{n-1}, S_{n+1}, \ldots, S_{m} \subset R$ under the localization morphism $R \longrightarrow S_{n}^{-1} R$.

It needs to be observed that, if the multiplicative subset $S_{j} \subset R$ is countable, then the multiplicative subset $S_{j}^{\prime} \subset R^{\prime}$ is countable; if $\operatorname{pd}_{R} S_{j}^{-1} R \leq 1$, then $\operatorname{pd}_{R^{\prime}} S_{j}^{\prime-1} R^{\prime} \leq 1$ by Lemma 5.1 (a); and if the $S_{j}$-torsion in $R$ is bounded, then the $S_{j}^{\prime}$-torsion in $R^{\prime}$ is bounded by Lemma 5.5. The $R^{\prime}$-module $\operatorname{Hom}_{R}\left(S_{n}^{-1} R, C\right)$ is $S_{J}^{\prime}$-weakly cotorsion for all $J \subset\{1, \ldots, n-1\}$ and an $S_{k}^{\prime}$-contramodule for all $n+1 \leq k \leq m$ by Lemma 5.1(b-c). Finally, for any subset of indices $J^{\prime} \subset\{1, \ldots, n-1\} \subset\{1, \ldots$, $n-1, n+1, \ldots, m\}$ and any collection $\mathbf{s}^{\prime}$ of elements $s_{n+1}^{\prime} \in S_{n+1}^{\prime}, \ldots, s_{m}^{\prime} \in S_{m}^{\prime}$, the $R$-algebra $R_{J^{\prime}, \mathbf{s}^{\prime}}^{\prime}$ is isomorphic to $R_{J, s}$, where $J=J^{\prime} \cup\{n\} \subset\{1, \ldots, n\} \subset\{1, \ldots, m\}$ is the related subset of indices and $\mathbf{s}$ is a collection of preimages $s_{n+1} \in S_{n+1}, \ldots$, $s_{m} \in S_{m}$ of the elements $s_{k}^{\prime} \in S_{k}^{\prime}, n+1 \leq k \leq m$.

The $R$-module $\Delta_{R, S_{n}}(C)$ is $S_{J}$-weakly cotorsion for all $J \subset\{1, \ldots, n-1\}$, since it is a quotient module of an $S_{J}$-weakly cotorsion $R$-module $C$ and $\operatorname{pd}_{R} S_{J}^{-1} R \leq 1$. It is also an $S_{n}$-contramodule by Lemma 3.1(b), and an $S_{k}$-contramodule for all $n+1 \leq k \leq m$ by Lemma $5.2(\mathrm{~b})$. So $\Delta_{R, S_{n}}(C)$ is an $S_{k}$-contramodule $R$-module for all $n \leq k \leq m$. The claim that the $R$-module $\Delta_{R, S_{n}}(C)$ is right 1-obtainable from $\mathrm{E}_{n}$ (in fact, even from $\mathrm{E}_{n-1}$ ) now follows from the induction assumption applied to the pair of integers $\left(n^{\prime}, m^{\prime}\right)=(n-1, m)$, the same ring $R^{\prime}=R$, and the same collection of multiplicative subsets $S_{1}^{\prime}=S_{1}, \ldots, S_{m}^{\prime}=S_{m}$.

The $R$-module $\operatorname{Hom}_{R}\left(S_{n}^{-1} R / R, C\right)$ is an $S_{n}$-contramodule by Lemma 4.2 and an $S_{k}$-contramodule for all $n+1 \leq k \leq m$ by Lemma 5.2(b). By Lemma 5.3, the 
$R$-module $\operatorname{Hom}_{R}\left(S_{n}^{-1} R / R, C\right)$ is simply right obtainable from modules over the quotient rings $R^{\prime \prime}=R /\left(s_{n} R+s_{n+1} R+\cdots+s_{m} R\right)$ for various combinations of elements $s_{n} \in S_{n}, s_{n+1} \in S_{n+1}, \ldots, s_{m} \in S_{m}$.

Denote by $S_{J}^{\prime \prime} \subset R^{\prime \prime}$ the images of the multiplicative subsets $S_{J} \subset R, \quad J \subset$ $\{1, \ldots, n-1\}$ in the ring $R^{\prime \prime}$. By Lemma 5.1(a), we have $\operatorname{pd}_{R^{\prime \prime}} S_{J}^{\prime \prime-1} R^{\prime \prime} \leq 1$. Therefore, by Lemma 2.6, all $R^{\prime \prime}$-modules are right 2-obtainable from $R^{\prime \prime}$-modules that are $S_{J}^{\prime \prime}$-weakly cotorsion for all $J \subset\{1, \ldots, n-1\}$. Applying Lemma 5.1(b), we see that all such $R^{\prime \prime}$-modules are $S_{J}$-weakly cotorsion as $R$-modules. Thus all $R$-modules annihilated by $s_{n} R+\cdots+s_{m} R$ are right 2-obtainable from $R$-modules annihilated by $s_{n} R+\cdots+s_{m} R$ that are $S_{J}$-weakly cotorsion for all $J \subset\{1, \ldots, n-1\}$. Any $R$-module annihilated by an element $s_{k} \in S_{k}$ is an $S_{k}$-contramodule.

Now we apply the induction assumption to the pair of integers $\left(n^{\prime}, m^{\prime}\right)=(n-1, m)$, the ring $R^{\prime}=R$, and the collection of multiplicative subsets $S_{1}^{\prime}=S_{1}, \ldots, S_{m}^{\prime}=S_{m}$ in order to conclude that all $R$-modules annihilated by $s_{n} R+\cdots+s_{m} R, s_{k} \in S_{k}$, are right 2-obtainable from $\mathrm{E}_{n-1}$. It follows that the $R$-module $\operatorname{Hom}_{R}\left(S_{n}^{-1} R / R, C\right)$ is also right 2-obtainable from $\mathrm{E}_{n-1}$.

This proves the "only if" assertion of the proposition. The "if" assertion holds for any collection of multiplicative subsets $S_{1}, \ldots, S_{m}$ in a commutative ring $R$. To prove as much, let $\mathrm{E} \subset R$-mod denote the class of all $R_{J, \mathbf{s}}$-modules, viewed as $R$-modules via the restriction of scalars, where $J \subset\{1, \ldots, m\}$ and $\mathbf{s}$ runs over all the collections of elements $s_{k} \in S_{k}, k \in K=\{1, \ldots, m\} \backslash J$.

By Lemma 4.3, for any $R$-module $E \in \mathrm{E}$ and any subset of indices $I \subset\{1, \ldots, m\}$, we have $\operatorname{Ext}_{R}^{i}\left(S_{I}^{-1} R, E\right)=0$ for all $i>0$, since $S_{I}^{-1} R$ is a flat $R$-module and

$$
R_{J, \mathbf{s}} \otimes_{R} S_{I}^{-1} R= \begin{cases}R_{J, \mathbf{s}} & \text { if } I \subset J, \\ 0, & \text { if } I \not \subset J\end{cases}
$$

is always a free $R_{J, \mathbf{s}}$-module (with 1 or 0 generators). It remains to apply Lemma 2.4 in order to conclude that $\operatorname{Ext}_{R}^{1}\left(S_{I}^{-1} R, C\right)=0$ for all $R$-modules $C$ right 1-obtainable from $\mathrm{E}$.

Proof of Theorem 1.10. The "only if" assertion holds for any collection of multiplicative subsets $S_{1}, \ldots, S_{m}$ in a commutative ring $R$. Indeed, an $R$-module is $\mathbf{S}^{\times}$-strongly flat if and only if it is a direct summand of a transfinitely iterated extension, in the sense of the inductive limit, of copies of the $R$-modules $S_{I}^{-1} R$, $I \subset\{1, \ldots, m\}$ [13, Corollary 6.14]. Now, for any subset $J \subset\{1, \ldots, m\}$ and any collection $\mathbf{s}$ of elements $s_{k} \in S_{k}, \quad k \in\{1, \ldots, m\} \backslash J$, the functor of extension of scalars $R_{J, \mathbf{s}} \otimes_{R}-: R-\bmod \longrightarrow R_{J, \mathbf{s}}-$ mod takes transfinitely iterated extensions (in the sense of the inductive limit) of flat $R$-modules to transfinitely iterated exensions of flat $R_{J, \mathbf{s}}$-modules, and the $R$-modules $S_{I}^{-1} R$ to free $R_{J, \mathbf{s}}$-modules (with 1 or 0 generators).

To prove the "if", denote, as above, by $\mathrm{E} \subset R$-mod the class of all $R_{J, \mathrm{~s}}$-modules (viewed as $R$-modules via the restriction of scalars). Let $F$ be a flat $R$-module such that the $R_{J, \mathbf{s}}$-module $R_{J, \mathbf{s}} \otimes_{R} F$ is projective for all subsets of indices $J \subset\{1, \ldots, m\}$ 
and all collections $\mathbf{s}$ of elements $s_{k} \in S_{k}, k \in\{1, \ldots, m\} \backslash J$. Then, by Lemma 4.3, we have $\operatorname{Ext}_{R}^{i}(F, E)=0$ for all $E \in \mathrm{E}$ and all $i>0$.

Applying Lemma 2.4, we see that $\operatorname{Ext}_{R}^{i}(F, C)=0$ for all $R$-modules $C$ right 1-obtainable from $\mathbf{E}$ and all $i>0$. According to Proposition 1.11, all $\mathbf{S}^{\times}$-weakly cotorsion $R$-modules are right 1-obtainable from E. Thus $\operatorname{Ext}_{R}^{1}(F, C)=0$ for all $\mathbf{S}^{\times}$-weakly cotorsion $R$-modules $C$, so $F$ is an $\mathbf{S}^{\times}$-strongly flat $R$-module.

\section{Finite-Dimensional Noetherian Rings with Countable Spectrum}

The aim of this section is to prove Theorems 1.14, 1.15, and 1.16. The essential idea of these arguments was already stated in Section 1.5; the following corollary, formulated in the notation of Section 1.4, summarizes it.

Corollary 6.1. Let $\mathbf{S}=\left\{S_{1}, \ldots, S_{m}\right\}$ be a finite collection of countable multiplicative subsets in a commutative ring $R$. Assume that, for any subset of indices $J \subset\{1, \ldots, m\}$ and any collection of elements $s_{k} \in S_{k}, k \in K=\{1, \ldots, m\} \backslash J$, the ring $R_{J, \mathbf{s}}$ is Artinian. Then all flat $R$-modules are $\mathbf{S}^{\times}$-strongly flat.

Proof. This is a corollary of Theorem 1.10. Since we assume the multiplicative subsets $S_{1}, \ldots, S_{m} \subset R$ to be countable, the condition that the projective dimension of the $R$-module $S_{J}^{-1} R$ does not exceed 1 for all $J \subset\{1, \ldots, m\}$ holds automatically. All flat modules over an Artinian commutative ring are projective, so our assumptions guarantee that all flat $R_{J, \mathbf{s}}$-modules are projective, and the assertion of the corollary follows immediately from Theorem 1.10.

Let $P$ be a (commutative) principal ideal domain and $R=P[x]$ be the ring of polynomials in one variable $x$ with coefficients in $P$. Changing the notation of Section 1.5 slightly, denote by $S \subset P[x]$ the multiplicative subset of all nonzero elements of $P$, viewed as elements of $P[x]$, and by $T \subset P[x]$ the multiplicative subset of all polynomials with content 1 .

Proposition 6.2. For any principal ideal domain $P$, and any two elements $s \in S$ and $t \in T$, the four rings $R / s R \otimes_{R} R / t R, \quad R / s R \otimes_{R} T^{-1} R, \quad S^{-1} R \otimes_{R} R / t R$, and $S^{-1} R \otimes_{R} T^{-1} R$ are Artinian.

Proof. The multiplicative subset $S T \subset P[x]$ consists of all the nonzero elements of the commutative domain $R=P[x]$, so $S^{-1} R \otimes_{R} T^{-1} R=(S T)^{-1} R$ is a field. Furthermore, denoting by $Q$ the field of fractions of the domain $P$, one has $S^{-1} R \otimes_{R} R / t R=$ $Q[x] / t Q[x]$, where $t$ is a nonzero polynomial in one variable $x$ over the field $Q$. This is obviously an Artinian ring. Finally, for any commutative ring $A$ with a finitely generated nilpotent ideal $\mathfrak{n} \subset A$, the ring $A$ is Artinian if and only if the $\operatorname{ring} A / \mathfrak{n}$ is. Besides, the direct sum of a finite number of Artinian rings is Artinian. This reduces the task of proving that the rings $R / s R \otimes_{R} R / t R$ and $R / s R \otimes_{R} T^{-1} R$ are Artinian to the case of a prime element $s \in P$. In this case, $k=P / s P$ is a field, and the ring $R / s R \otimes_{R} T^{-1} R$ is isomorphic to the field of rational functions $k(x)$, since 
every unital polynomial $f(x)=x^{n}+f_{n-1} x^{n-1}+\cdots+f_{0}$ in the variable $x$ with the coefficients in $k$ can be lifted to a unital polynomial in $x$ with the coefficients in $P$, which belongs to $T$. On the other hand, any polynomial $t=t(x) \in T$ reduces to a nonzero polynomial $g(x) \in k[x]$ modulo $s$, so the ring $R / s R \otimes_{R} R / t R$ is isomorphic to the quotient ring $k[x] / g k[x]$, which is Artinian.

Proof of Theorem 1.14. When the principal ideal domain $P$ is countable, so are both the multiplicative subsets $S_{1}=S$ and $S_{2}=T \subset R=P[x]$. According to Proposition 6.2, all the rings $R_{J, \mathbf{s}}, J \subset\{1,2\}$, are Artinian, so Corollary 6.1 is applicable.

Remark 6.3. A commutative algebra $R^{\prime}$ over a commutative ring $R$ is said to be finite (over $R$ ) if it is finitely generated as an $R$-module. Let $P$ be a countable PID and $R^{\prime}$ be a finite commutative algebra over the ring $R=P[x]$. Denote by $S_{1}^{\prime}=S^{\prime}$ and $S_{2}^{\prime}=T^{\prime} \subset R^{\prime}$ the images of our two multiplicative subsets $S_{1}=S$ and $S_{2}=T \subset P[x]$ in the ring $R^{\prime}$. Then, for any elements $s^{\prime} \in S^{\prime}$ and $t^{\prime} \in T^{\prime}$, the four rings $R^{\prime} / s^{\prime} R^{\prime} \otimes_{R^{\prime}} R^{\prime} / t^{\prime} R^{\prime}, \quad R^{\prime} / s^{\prime} R^{\prime} \otimes_{R^{\prime}} T^{\prime-1} R^{\prime}, \quad S^{\prime-1} R^{\prime} \otimes_{R^{\prime}} R^{\prime} / t^{\prime} R^{\prime}$, and $S^{\prime-1} R^{\prime} \otimes_{R^{\prime}} T^{\prime-1} R^{\prime}$ are Artinian, because the similar rings related to the multiplicative subsets $S$ and $T \subset R$ are Artinian by Proposition 6.2 and a commutative ring finite over an Artinian commutative ring in Artinian.

This provides an elementary explicit construction of pairs of multiplicative subsets $\mathbf{S}^{\prime}=\left\{S_{1}^{\prime}, S_{2}^{\prime}\right\}$ in some countable Noetherian commutative rings $R^{\prime}$ of Krull dimension 2 such that all flat $R^{\prime}$-modules are $\mathbf{S}^{\prime \times}$-strongly flat. In particular, if $R^{\prime}$ is a finitely generated commutative algebra of transcendence degree ( = Krull dimension) 2 over a countable field $k$, then, by the Noether normalization lemma, one can find two elements $x, y \in R^{\prime}$ such that $R^{\prime}$ is finite over its $k$-subalgebra generated by $x$ and $y$, which is the polynomial algebra $k[x, y] \subset R^{\prime}$. Applying the above construction to the principal ideal domain $P=k[y]$ and the $\operatorname{ring} R=P[x]=k[x, y]$, we obtain a pair of multiplicative subsets $S_{1}^{\prime}, S_{2}^{\prime} \subset R^{\prime}$ with the desired properties.

Let $R$ be a commutative ring, $S \subset R$ be a multiplicative subset, and $\mathfrak{p} \varsubsetneqq \mathfrak{q}$ be two prime ideals in $R$, one of which is properly contained in the other one. We will say that the multiplicative subset $S$ distinguishes $\mathfrak{p}$ from $\mathfrak{q}$ if $\mathfrak{p} \cap S=\varnothing$ and $\mathfrak{q} \cap S \neq \varnothing$. We will say that a collection of multiplicative subsets $S_{1}, \ldots, S_{m} \subset R$ distinguishes $\mathfrak{p}$ from $\mathfrak{q}$ if there exists $1 \leq j \leq m$ such that $S_{j}$ distinguishes $\mathfrak{p}$ from $\mathfrak{q}$.

Lemma 6.4. Let $R$ be a Noetherian commutative ring and $S_{1}, \ldots, S_{m} \subset R$ be a collection of multiplicative subsets in $R$. Then the ring $R_{J, \mathbf{s}}$ is Artinian for every subset of indices $J \subset\{1, \ldots, m\}$ and every collection of elements $s_{k} \in S_{k}, k \in$ $\{1, \ldots, m\} \backslash J$ if and only if for every pair of prime ideals $\mathfrak{p} \varsubsetneqq \mathfrak{q}$ in $R$ there exists $1 \leq j \leq m$ for which $\mathfrak{p} \cap S_{j}=\varnothing$, while $\mathfrak{q} \cap S_{j} \neq \varnothing$.

Proof. For any commutative ring $R$ with a collection of multiplicative subsets $S_{1}, \ldots$, $S_{m} \subset R$, and any $J \subset\{1, \ldots, m\}$ and $s_{k} \in S_{k}$, the map of spectra $\operatorname{Spec} R_{J, \mathbf{s}} \longrightarrow$ Spec $R$ induced by the commutative ring homomorphism $R \longrightarrow R_{J, \mathbf{s}}$ identifies the spectrum of the ring $R_{J, \mathbf{s}}$ with the subset in $\operatorname{Spec} R$ consisting of all the prime ideals $\mathfrak{p} \subset R$ such that $\mathfrak{p} \cap S_{j}=\varnothing$ for all $j \in J$ and $s_{k} \in \mathfrak{p}$ for all $k \notin J$. If the collection 
of multiplicative subsets $S_{1}, \ldots, S_{m}$ distinguishes all the pairs of prime ideals one of which is properly contained in the other one in the ring $R$, the above conditions cannot be simultaneously satisfied for two such prime ideals $\mathfrak{p} \mp \mathfrak{q} \subset R$. Hence the Krull dimension of the ring $R_{J, \mathbf{s}}$ is equal to 0 . If the ring $R$ is Noetherian, then so is the ring $R_{J, \mathbf{s}}$; and it follows that $R_{J, \mathbf{s}}$ is Artinian.

Conversely, let $\mathfrak{p} \varsubsetneqq \mathfrak{q}$ be a pair of prime ideals in $R$ not distinguished by the multiplicative subsets $S_{1}, \ldots, S_{m}$. Denote by $J$ the set of all indices $1 \leq j \leq m$ for which $\mathfrak{q} \cap S_{j}=\varnothing$. For every $k \in\{1, \ldots, m\} \backslash J$, the intersection $\mathfrak{p} \cap S_{k}$ is nonempty, so the exists an element $s_{k} \in \mathfrak{p} \cap S_{k}$. Consider the related ring $R_{J, \mathbf{s}}$. Then both the prime ideals $\mathfrak{p}$ and $\mathfrak{q} \subset R$ belong to the image of the map $\operatorname{Spec} R_{J, \mathbf{s}} \longrightarrow \operatorname{Spec} R$, so they correspond to a pair of prime ideals one of which is properly contained in the other one in the ring $R_{J, \mathbf{s}}$. Consequently, the ring $R_{J, \mathbf{s}}$ has positive Krull dimension and is not Artinian.

The next lemma is a simple elementary version of the one following after it.

Lemma 6.5. Let $R$ be a Noetherian ring of Krull dimension 1 . Then there exists a multiplicative subset $S \subset R$ such that all the rings $R / s R, s \in S$, and $S^{-1} R$ are Artinian. If the spectrum of $R$ is countable, one can choose $S$ to be a countable multiplicative subset in $R$.

Proof. The point is that the set of all minimal prime ideals in a Noetherian commutative ring is finite. To obtain just some multiplicative subset $S \subset R$ for which the rings $R / s R$ and $S^{-1} R$ are Artinian, it suffices to set $S=R \backslash \bigcup_{i=1}^{k} \mathfrak{q}_{i}$, where $\mathfrak{q}_{i}$ are the minimal prime ideals in $R$. Then, by prime avoidance, for any prime ideal $\mathfrak{p} \subset R$ of height 1 there exists an element $s \in \mathfrak{p} \cap S$, so the multiplicative subset $S \subset R$ distinguishes all the pairs of prime ideals one of which is properly contained in the other one in the ring $R$ (cf. [23, Section 13]). When $\operatorname{Spec} R$ is countable, one can choose, for every prime ideal $\mathfrak{p} \subset R$ of height 1 , an element $s \in R$ not belonging to any of the minimal prime ideals, and set $S$ to be the multiplicative subset generated by all the elements $s$ so obtained. Then $S$ is countable and all the pairs of prime ideals one of which is properly contained in the other one in $R$ are still distinguished by $S$, so the rings $R / s R, s \in S$, and $S^{-1} R$ are Artinian.

Lemma 6.6. Let $R$ be a Noetherian commutative ring of Krull dimension 2 with countable spectrum. Then there exists a pair of countable multiplicative subsets $S$ and $T \subset R$ such that, for any two elements $s \in S$ and $t \in T$, the four rings $R / s R \otimes_{R} R / t R$, $R / s R \otimes_{R} T^{-1} R, \quad S^{-1} R \otimes_{R} R / t R$, and $S^{-1} R \otimes_{R} T^{-1} R$ are Artinian.

Proof. Let $\mathfrak{p}_{1}, \mathfrak{p}_{2}, \ldots$ be all the prime ideals of the ring $R$, numbered by the positive integers in an arbitrary order. We proceed by induction, constructing two nondecreasing sequences of finite subsets of elements $S_{0}^{\prime} \subset S_{1}^{\prime} \subset S_{2}^{\prime} \subset \cdots \subset R$ and $T_{0}^{\prime} \subset T_{1}^{\prime} \subset T_{2}^{\prime} \subset \cdots \subset R$ in the following way.

Throughout the construction, the following conditions will be always satisfied. Prime ideals of height 0 in $R$ do not intersect the subsets $S_{n}^{\prime}$ and $T_{n}^{\prime} \subset R$. A prime ideal of height 1 may intersect either $S_{n}^{\prime}$, or $T_{n}^{\prime}$, but not both. Prime ideals of height 2 are allowed to intersect both $S_{n}^{\prime}$ and $T_{n}^{\prime}$. 
For the purposes of this proof, given a finite subset $U=S_{n}^{\prime}$ or $T_{n}^{\prime} \subset R$, we will say that a prime ideal $\mathfrak{p} \subset R$ of height 1 is a $U$-prime if $\mathfrak{p}$ intersects $U$. Since the primes of height 0 do not intersect $U$, such a prime $\mathfrak{p}$ is a minimal prime over the principal ideal $(u) \subset R$ generated by some element $u \in U$. Since the set $U$ is finite and the set of all minimal prime ideals in a Noetherian commutative ring is finite, the set of all $U$-primes in $R$ will be always finite. (Notice that, by Krull's Hauptidealsatz, all minimal primes over a principal ideal in $R$ have height at most 1 ; but we do not need to use this result, as all our " $U$-primes" have height 1 by definition.)

To begin with, set $S_{0}^{\prime}=\varnothing=T_{0}^{\prime}$. For any $n \geq 1$, suppose that the subsets $S_{n-1}^{\prime}$ and $T_{n-1}^{\prime} \subset R$ have been constructed already, and consider the prime ideal $\mathfrak{p}_{n} \subset R$. If $\mathfrak{p}_{n}$ is a prime ideal of height 0 , we set $S_{n}^{\prime}=S_{n-1}^{\prime}$ and $T_{n}^{\prime}=T_{n-1}^{\prime}$.

If $\mathfrak{p}_{n}$ is a prime ideal of height 1 , it may intersect one of the two subsets $S_{n-1}^{\prime}$ and $T_{n-1}^{\prime}$, or it may intersect neither of them. In the former case, we set $S_{n}^{\prime}=S_{n-1}^{\prime}$ and $T_{n}^{\prime}=T_{n-1}^{\prime}$. In the latter case, we use prime avoidance to find an element $s \in \mathfrak{p}_{n}$ not belonging to any of the $T_{n-1}^{\prime}$-primes and any of the primes of height 0 in $R$. Set $S_{n}^{\prime}=S_{n-1}^{\prime} \cup\{s\}$ and $T_{n}^{\prime}=T_{n-1}$.

If $\mathfrak{p}_{n}$ is a prime ideal of height 2 , it may intersect both the subsets $S_{n-1}^{\prime}$ and $T_{n-1}^{\prime} \subset R$, or only one of them, or neither one. In any case, we construct the sets $S_{n}^{\prime}$ and $T_{n}^{\prime}$ so that $\mathfrak{p}_{n}$ intersects both of them. Specifically, if $\mathfrak{p}_{n} \cap S_{n-1}^{\prime}=\varnothing$, we find an element $s \in \mathfrak{p}_{n}$ not belonging to any of the $T_{n-1}^{\prime}$-primes and any of the primes of height 0 in $R$, and set $S_{n}^{\prime}=S_{n-1}^{\prime} \cup\{s\}$. If $\mathfrak{p}_{n} \cap S_{n-1}^{\prime} \neq \varnothing$, we set $S_{n}^{\prime}=S_{n-1}^{\prime}$. Then, if $\mathfrak{p}_{n} \cap T_{n-1}^{\prime}=\varnothing$, we find an element $t \in \mathfrak{p}_{n}$ not belonging to any of the $S_{n}^{\prime}$-primes and any of the primes of height 0 in $R$, and set $T_{n}^{\prime}=T_{n-1}^{\prime} \cup\{t\}$. If $\mathfrak{p}_{n} \cap T_{n-1}^{\prime} \neq \varnothing$, we set $T_{n}^{\prime}=T_{n-1}^{\prime}$.

After the whole inductive process has been finished, we set $S^{\prime}=\bigcup_{n=1}^{\infty} S_{n}^{\prime}$ and $T^{\prime}=\bigcup_{n=1}^{\infty} T_{n}^{\prime}$, and observe that any prime ideal $\mathfrak{p} \subset R$ of height $h$ intersects exactly $h$ of the two subsets $S^{\prime}$ and $T^{\prime} \subset R$. Finally, we set $S \subset R$ to be the multiplicative subset generated by $S^{\prime}$ and $T \subset R$ to be the multiplicative subset generated by $T^{\prime}$ in $R$, and again observe that any prime ideal of height $h$ in $R$ intersects exactly $h$ of the two multiplicative subsets $S$ and $T \subset R$.

Applying Lemma 6.4, we conclude that the pair of countable multiplicative subsets $S$ and $T \subset R$ has the desired properties.

Proof of Theorem 1.15. Denote the two multiplicative subsets $S$ and $T$ constructed in Lemma 6.6 by $S_{1}=S$ and $S_{2}=T$, and apply Corollary 6.1.

Remark 6.7. One might wish to extend the construction of Lemma 6.6 to Noetherian rings $R$ of Krull dimension 3 with countable spectrum by producing a collection of three multiplicative subsets $S, T$, and $U \subset R$ such that any prime ideal of height $h$ in $R$ intersects exactly $h$ of them. However, the most straightforward attempt to do so breaks down on the following problem. Arguing as in the proof of Lemma 6.6, suppose that we have a prime ideal $\mathfrak{p}_{n} \subset R$ of height 1 which does not intersect any of the three sets $S_{n-1}^{\prime}, T_{n-1}^{\prime}$, and $U_{n-1}^{\prime}$. We need to add an element of the ideal $\mathfrak{p}_{n}$ to one of these three sets in order to produce a new set $S_{n}^{\prime}, T_{n}^{\prime}$, or $U_{n}^{\prime}$ that would interstect $\mathfrak{p}_{n}$. The problem occurs when the prime ideal $\mathfrak{p}_{n}$ is contained in three 
prime ideals of height 2 , say $\mathfrak{q}^{\prime}, \mathfrak{q}^{\prime \prime}$, and $\mathfrak{q}^{\prime \prime \prime}$, such that $\mathfrak{q}^{\prime}$ intersects $S_{n-1}^{\prime}$ and $T_{n-1}^{\prime}$, while $\mathfrak{q}^{\prime \prime}$ intersects $S_{n-1}^{\prime}$ and $U_{n-1}^{\prime}$, and at the same time $\mathfrak{q}^{\prime \prime \prime}$ intersects $T_{n-1}^{\prime}$ and $U_{n-1}^{\prime}$. Then, into whichever of the three sets $S_{n}^{\prime}, T_{n}^{\prime}$, or $U_{n}^{\prime}$ we decide to include an element of the prime ideal $\mathfrak{p}_{n}$, the condition that any prime ideal of height 2 intersects at most two of these three sets would be violated.

Another approach might be to do several waves of the induction process, treating all the prime ideals of height 1 before starting with the prime ideals of height 2 . But then the sets $S^{\prime}, T^{\prime}$, and $U^{\prime}$ produced after an infinite number of such steps become infinite themselves, and the argument with prime avoidance, based on there being only a finite number of prime ideals of height 1 intersecting either of these sets, would no longer work (of course). Yet another approach, which we successfully develop below, is to use more than $d$ multiplicative subsets for a Noetherian ring of Krull dimension $d$ with countable spectrum.

In fact, more than $d$ multiplicative subsets for a Noetherian ring of Krull dimension $d$ are necessary in some cases. Let $R=k[x, y, z]$ be the ring of polynomials in three variables over a countable field $k$, or alternatively, $R=\mathbb{Z}[x, y]$ be the ring of polynomials in two variables with integer coefficients. Then the intersection of any finite set of prime ideals of height 2 in $R$ contains infinitely many primes of height 1 [19, Corollary 11], and in particular, the intersection of any three prime ideals of height 2 contains a prime ideal of height 1 . For this reason, one cannot find 3 multiplicative subsets in $R$ such that any prime ideal of height $h$ intersects exactly $h$ of them. So the result of Proposition 6.9 below, providing 4 multiplicative subsets in a Noetherian ring of Krull dimension 3 with countable spectrum, is really the best one could hope for in these cases.

In the sequel, when we say that "a collection of multiplicative subsets $S_{1}, \ldots$, $S_{m} \subset R$ in a commutative ring $R$ distinguishes all prime ideals belonging to a certain set of primes $P \subset$ Spec $R$ from all prime ideals belonging to a certain other set of primes $Q \subset \operatorname{Spec} R$ ", we mean that every pair of prime ideals $\mathfrak{p} \varsubsetneqq \mathfrak{q}$ with $\mathfrak{p}$ and $\mathfrak{q}$ belonging to the respective sets of prime ideals in $R$ is distinguished by the collection of multiplicative subsets $S_{1}, \ldots, S_{m} \subset R$. So, "distinguishing prime ideals" always means distinguishing pairs of prime ideals one of which is properly contained in the other one.

Lemma 6.8. Let $R$ be a Noetherian commutative ring with countable spectrum, and let $l \geq 2$ be an integer. Then there exists a collection of $l$ multiplicative subsets $S_{1}, \ldots, S_{l} \subset R$ distinguishing all the prime ideals of height $l$ and $l-1$ in $R$ from each other, and from all the prime ideals of smaller height.

Proof. The assertion of the lemma means that, for every pair of prime ideals $\mathfrak{p} \varsubsetneqq \mathfrak{q}$ in $R$ such that the height of $\mathfrak{q}$ is equal to $l$ or $l-1$, there exists $1 \leq j \leq l$ for which $S_{j} \cap \mathfrak{p}=\varnothing$ and $S_{j} \cap \mathfrak{q} \neq \varnothing$.

The proof is similar to that of Lemma 6.6. Let $\mathfrak{p}_{1}, \mathfrak{p}_{2}, \ldots$ be all the prime ideals of height $l$ or $l-1$ in the ring $R$, numbered by the positive integers in an arbitrary order. We proceed by induction, constructing $l$ nondecreasing sequences of finite subsets of 
elements $S_{j, 0}^{\prime} \subset S_{j, 1}^{\prime} \subset S_{j, 2}^{\prime} \subset \cdots, \quad 1 \leq j \leq l$ in the ring $R$ in the way described below.

Throughout the construction, the following condition will be always satisfied. Any prime ideal of height $h, 0 \leq h \leq l$ in the $\operatorname{ring} R$ may intersect at most $h$ of the $l$ finite sets $S_{1, n}^{\prime}, \ldots, S_{l, n}^{\prime} \subset R$ (but not more).

We will denote the collections of $l$ sets $S_{1, n}^{\prime}, \ldots, S_{l, n}^{\prime}$ by $\mathbf{S}_{n}^{\prime}$ for brefity, and say that a prime ideal $\mathfrak{p} \subset R$ of height $h$ is an $\mathbf{S}_{n}^{\prime}$-prime if $\mathfrak{p}$ intersects $h$ of the $l$ sets $S_{1, n}^{\prime}, \ldots, S_{l, n}^{\prime}$. Since a prime ideal of height less than $h$ cannot intersect $h$ of these sets by assumption, such a prime $\mathfrak{p}$ is a minimal prime over the ideal generated by some collection of $h$ elements $s_{j} \in S_{j, n}^{\prime}, \quad j \in J$, where $J \subset\{1, \ldots, l\}$ is a subset of indices of the cardinality $|J|=h$. Since all the sets $S_{1, n}^{\prime}, \ldots, S_{l, n}^{\prime}$ are finite and the set of all minimal prime ideals in a Noetherian commutative ring is finite, the set of all $\mathbf{S}_{n}^{\prime}$-primes in $R$ will be always finite.

To begin with, set $S_{j, 0}^{\prime}=\varnothing$ for all $1 \leq j \leq l$. For any $n \geq 1$, suppose that the subsets $S_{j, n-1}^{\prime} \subset R, 1 \leq j \leq l$ have been constructed already; and consider the minimal element $i_{n}$ in the set of all positive integers $i$ such that the prime ideal $\mathfrak{p}_{i} \subset R$ is not an $\mathbf{S}_{n-1}^{\prime}$-prime.

Since $\mathfrak{p}_{i_{n}}$ is a prime ideal of height $l$ or $l-1$, there exists an index $k, 1 \leq k \leq l$ such that $\mathfrak{p}_{i_{n}} \cap S_{k, n-1}^{\prime}=\varnothing$. By prime avoidance, we can find an element $s \in \mathfrak{p}_{i_{n}}$ not belonging to any of the $\mathbf{S}_{n-1}^{\prime}$-primes of height $\leq l-1$ in $R$. Then we set $S_{k, n}^{\prime}=$ $S_{k, n-1}^{\prime} \cup\{s\}$ and $S_{j, n}^{\prime}=S_{j, n-1}^{\prime}$ for all $j=1, \ldots, k-1, k+1, \ldots, l$.

The sequence of integers $i_{n}$ is nondecreasing, $1 \leq i_{1} \leq i_{2} \leq i_{2} \leq i_{3} \leq \ldots$; it may visit a given integer $i$ more than once, but at most $l$ times. If for a certain $n \geq 1$ it turns out that all primes of height $l$ and $l-1$ are $\mathbf{S}_{n-1}^{\prime}$-primes already, we stop the induction at this point and set $S_{j, n-1}^{\prime}=S_{j, n}^{\prime}=S_{j, n+1}^{\prime}=\cdots$ for all $1 \leq j \leq l$. Otherwise, the construction proceeds for all $n \geq 1$.

After the whole inductive process has been finished, we set $S_{j}^{\prime}=\bigcup_{n=1}^{\infty} S_{j, n}^{\prime}$ for all $j=1, \ldots, l$, and observe that any prime ideal $\mathfrak{p} \subset R$ of height $h \leq l-2$ intersects at most $h$ of the $l$ subsets $S_{1}^{\prime}, \ldots, S_{l}^{\prime} \subset R$, while any prime ideal $\mathfrak{q} \subset R$ of height $h=l$ or $l-1$ intersects exactly $h$ of these $l$ subsets. Finally, we set $S_{j} \subset R, 1 \leq j \leq l$, to be the multiplicative subset generated by $S_{j}^{\prime}$, and again observe that any prime ideal of height $h \leq l-2$ in $R$ intersects at most $h$ of the multiplicative subsets $S_{1}, \ldots$, $S_{l} \subset R$, while any prime ideal of height $h=l$ or $l-1$ in $R$ intersects exactly $h$ of these $l$ multiplicative subsets.

We recall the notation $\mu: \mathbb{Z}_{\geq 0} \longrightarrow \mathbb{Z}_{\geq 0}$ for the function taking a nonnegative integer $d$ to the nonnegative integer $\mu(d)=d+(d-2)+(d-4)+\cdots+(d-2 k)$, where $d-2 k=0$ or 1 (see Section 1.5).

Proposition 6.9. Let $R$ be a Noetherian commutative ring of finite Krull dimension $d$ with countable spectrum. Then there exists a collection of $m=\mu(d)$ countable multiplicative subsets $S_{1}, \ldots, S_{m} \subset R$ such that, for every pair of prime ideals $\mathfrak{p} \varsubsetneqq \mathfrak{q}$ in $R$ there exists $1 \leq j \leq m$ for which $\mathfrak{p} \cap S_{j}=\varnothing$, while $\mathfrak{q} \cap S_{j} \neq \varnothing$. 
Proof. If $d=0$, then there are no pairs of prime ideals one of which is properly contained in the other one in the ring $R$, so $m=0$ and an empty collection of multiplicative subsets in $R$ is sufficient. If $d=1$, one has $m=1$ and the one countable multiplicative subset $S \subset R$ constructed in Lemma 6.5 is sufficient. If $d=2$, one has $m=2$ and there is a pair of multiplicative subsets $S$ and $T \subset R$ constructed in Lemma 6.6.

Generally for an arbitrary integer $d \geq 0$, let $k \geq 0$ be an integer such that $d-2 k=0$ or 1 . We apply Lemma $6.8 k$ times to the same $\operatorname{ring} R$ with the parameter $l=d$, $l=d-2, \ldots, l=d-2 k+2$. This produces the total of $m^{\prime}=d+(d-2)+\cdots+(d-2 k+2)$ countable multiplicative subsets $S_{1}, \ldots, S_{m^{\prime}} \subset R$.

The first $d$ of these $m^{\prime}$ multiplicative subsets distinguish prime ideals of height $d$ and $d-1$ from each other and from prime ideals of lower height; the next $d-2$ of these multiplicative subsets distinguish prime ideals of height $d-2$ and $d-3$ from each other and from prime ideals of lower height, etc. The last $d-2 k+2=2$ or 3 of these $m^{\prime}$ multiplicative subsets distinguish prime ideals of height $d-2 k+2$ and $d-2 k+1$ from each other and from prime ideals of height $d-2 k$ and lower.

If $d$ is even, then $d-2 k=0, m=m^{\prime}$, and we are done. If $d$ is odd, then $d-2 k=1, m=m^{\prime}+1$, and we still need one more multiplicative subset $S_{m} \subset R$ to distinguish prime ideals of height 1 from prime ideals of height 0 in $R$. The argument here repeats the one in the proof of Lemma 6.5. For every prime ideal $\mathfrak{p}$ of height 1 in $R$, we use prime avoidance to choose an element $s \in \mathfrak{p}$ not belonging to any of (the finite set of) prime ideals of height 0 . Then set $S_{m} \subset R$ to be the multiplicative subset generated by all the elements $s \in R$ so obtained.

Proof of Theorem 1.16. We apply Proposition 6.9 to produce a collection of $m=\mu(d)$ multiplicative subsets $S_{1}, \ldots, S_{m} \subset R$ distinguishing all the prime ideals in $R$. Then, by Lemma 6.4 , all the rings $R_{J, \mathbf{s}}$ are Artinian. According to Corollary 6.1, it follows that all flat $R$-modules are $\mathbf{S}^{\times}$-strongly flat.

\section{Contramodule Approximation Sequences}

The aim of this section is to prepare ground for the proofs of Main Lemmas 1.22 and 1.24 in the next Section 8. The constructions of contramodule approximation sequences in this section generalize those in [27, Sections 5.2-5.3].

We refer to [27, Section 5.1] and the references therein for a general discussion of cotorsion theories in abelian categories, including, first of all, the definitions of a cotorsion theory (or pair), the approximation sequences and a complete cotorsion theory, and also a hereditary complete cotorsion theory.

Specifically, we are interested in what we call the flat and the quite flat cotorsion theories in the abelian category of $S$-contramodule $R$-modules $R$-mod $\operatorname{m-ctra}_{\text {, }}$, where $R$ is a commutative ring and $S \subset R$ is a countable multiplicative subset (see Lemma 3.1(a)). Our constructions of these cotorsion theories will require making some assumptions on the ring $R$ and/or the multiplicative subset $S$. 
7.1. Flat cotorsion theory in $S$-contramodule $R$-modules for a Noetherian commutative ring $R$. We refer to Section 1.1 of the introduction and the paper [9] for the definition of an (Enochs) cotorsion R-module. In this section, as well as below, we will be particularly interested in $S$-contramodule $R$-modules that are flat, cotorsion, etc. as $R$-modules. To emphasize this aspect, we will call such $S$-contramodule $R$-modules $R$-flat, $R$-cotorsion, etc.

The aim of this section is to prove the following theorem (which generalizes [27, Theorem 5.2]).

Theorem 7.1. Let $R$ be a Noetherian commutative ring and $S \subset R$ be a countable multiplicative subset. Then the pair of full subcategories ( $R$-flat $S$-contramodule $R$-modules, $R$-cotorsion $S$-contramodule $R$-modules) is a hereditary complete cotorsion theory in the abelian category $R-\bmod _{S \text {-ctra }}$.

Let $R$ be a commutative ring and $S \subset R$ be a multiplicative subset. We refer to Section 4 for the discussion of $S$-divisible and $S$-h-divisible $R$-modules.

Lemma 7.2. Let $R$ be a commutative ring and $S \subset R$ be a countable multiplicative subset. Then an $R$-module is $S$-divisible if and only if it is $S$-h-divisible.

Proof. This well-known fact has a straightforward proof. One observes that, in the notation of Section 3.1, the $R$-module $S^{-1} R$ is the inductive limit of the inductive system of $R$-modules $R \stackrel{s_{1}}{\longrightarrow} R \stackrel{s_{2}}{\longrightarrow} R \stackrel{s_{3}}{\longrightarrow} \cdots$ (cf. [24, proof of Lemma 1.9]).

Now let $M$ be an $S$-divisible $R$-module and $x \in M$ be an element. Set $x_{0}=x$ and, proceeding by induction, choose elements $x_{1}, x_{2}, \ldots \in M$ such that $s_{n} x_{n}=x_{n-1}$ for all $n \geq 1$. Then there exists a unique $R$-module morphism $S^{-1} R \longrightarrow M$ taking the element $t_{n}^{-1} \in S^{-1} R$ to the element $x_{n} \in M$ for all $n \geq 0$.

The next three lemmas form a version of the theory developed in [21, Section B.9] and [23, Section 10] (see also the paper [33]). We refer to Section 3 for the definition of the $S$-completion functor $\Lambda_{R, S}$, as well as for the definition of a partial order on the set $S$ and the related discussion.

Lemma 7.3. Let $R$ be a Noetherian commutative ring, $S \subset R$ be a multiplicative subset, $K \subset L$ be a finitely generated $R$-module and its submodule, and $F$ be an $R$-module. Then the natural map between the projective limits

$$
\lim _{s \in S} K / s K \otimes_{R} F \longrightarrow \lim _{s \in S} K /(K \cap s L) \otimes_{R} F
$$

is an isomorphism.

Proof. For any $S$-indexed projective system of $R$-modules $\left(M_{s}\right)_{s \in S}$, there is a natural isomorphism of projective limits

$$
\lim _{s \in S} M_{s}=\lim _{s \in S} \lim _{n \geq 1} M_{s^{n}} .
$$

Thus it suffices to show that the natural map

$$
\lim _{n \geq 1} K / s^{n} K \otimes_{R} F \longrightarrow \lim _{n \geq 1} K /\left(K \cap s^{n} L\right) \otimes_{R} F
$$


is an isomorphism for every fixed $s \in S$. Now, according to the Artin-Rees Lemma applied to the pair of embedded finitely generated $R$-modules $K \subset L$ and the principal ideal $(s) \subset R$ generated by the element $s \in R$, there exists an integer $m \geq 1$ such that $K \cap s^{n} L=s^{n-m}\left(K \cap s^{m} L\right)$ for every $n \geq m$. Hence $s^{n} K \subset K \cap s^{n} L \subset s^{n-m} K$, implying the desired isomorphism of projective limits over $n$.

Lemma 7.4. Let $R$ be a Noetherian commutative ring, $S \subset R$ be a countable multiplicative subset, and $F$ be an $R$-module such that the $R / s R$-module $F / s F$ is flat for all $s \in S$. Then the $R$-module $\Lambda_{R, S}(F)=\lim _{s \in S} F / s F$ is flat.

Proof. Consider the functor $M \longmapsto \lim _{s \in S} M \otimes_{R} F / s F$ acting from the category of finitely generated $R$-modules to the category of $R$-modules. Let us show that this functor is exact.

Indeed, for any short exact sequence of finitely generated $R$-modules $0 \longrightarrow K \longrightarrow$ $L \longrightarrow M \longrightarrow 0$ there are short exact sequences of $R / s R$-modules $0 \longrightarrow K \cap s^{n} L \longrightarrow$ $s^{n} L \longrightarrow s^{n} M \longrightarrow 0$. Applying the functor $-\otimes_{R} F$ preserves exactness of these short exact sequences, since $F / s F$ is a flat $R / s R$ module. The passage to the projective limits over $s \in S$ preserves exactness of the resulting short exact sequences of tensor products, because these are countable filtered projective limits of surjective maps. It remains to take into account Lemma 7.3.

Furthermore, for any finitely generated $R$-module $M$ we have a natural $R$-module morphism

$$
M \otimes_{R} \lim _{s \in S} F / s F \longrightarrow \lim _{s \in S} M \otimes_{R} F / s F
$$

which is clearly an isomorphism for finitely generated free $R$-modules $M$. Both the functors being right exact on the category of finitely generated $R$-modules $M$, it follows that the morphism is an isomorphism for all such $M$ and the functor $M \longmapsto M \otimes_{R} \Lambda_{R, s}(F)$ is exact on the category of finitely gnerated $R$-modules.

We recall that, for any countable multiplicative subset $S$ in a commutative ring $R$, the natural $R$-module morphism $\Delta_{R, S}(M) \longrightarrow \Lambda_{R, S}(M)$ is surjective for all $R$-modules (see Lemma 3.2).

Lemma 7.5. Let $R$ be a Noetherian commutative ring, $S \subset R$ be a countable multiplicative subset, and $F$ be an $R$-module such that the $R$-module $F / s F$ is flat for all $s \in S$. Then the natural $R$-module map $\Delta_{R, S}(F) \longrightarrow \Lambda_{R, S}(F)$ is an isomorphism.

Proof. Denote by $K$ the kernel of the natural map $\Delta_{R, S}(F) \longrightarrow \Lambda_{R, S}(F)$; so we have a short exact sequence of $R$-modules $0 \longrightarrow K \longrightarrow \Delta_{R, S}(F) \longrightarrow \Lambda_{R, S}(F) \longrightarrow 0$. Applying the functor $R / s R \otimes_{R}$ - and taking into account the fact that the $R$-module $\Lambda_{R, S}(F)$ is flat by Lemma 7.4, we get a short exact sequence of $R / s R$-modules

$$
0 \longrightarrow K / s K \longrightarrow \Delta_{R, S}(F) / s \Delta_{R, S}(F) \longrightarrow \Lambda_{R, S}(F) / s \Lambda_{R, S}(F) \longrightarrow 0 .
$$

Now we have $\Delta_{R, S}(F) / s \Delta_{R, S}(F)=F / s F$ by $\left[24\right.$, Lemma 1.11] and $\Lambda_{R, S}(F) / s \Lambda_{R, S}(F)$ $=F / s F$ by $[24$, Proposition $2.2(\mathrm{~b})$ and Theorem $2.3(\mathrm{i}) \Leftrightarrow(\mathrm{iv})]$, hence it follows that $K / s K=0$ and $K=s K$. As this holds for all $s \in S$, we can apply Lemma 7.2 and conclude that the natural morphism $\operatorname{Hom}_{R}\left(S^{-1} R, K\right) \longrightarrow K$ is surjective. 
On the other hand, both $\Delta_{R, S}(F)$ and $\Lambda_{R, S}(F)$ are $S$-contramodule $R$-modules (see Lemma 3.1(b) and [24, Lemma 2.1(a)]), hence $K$ is an $S$-contramodule $R$-module and $\operatorname{Hom}_{R}\left(S^{-1} R, K\right)=0$. Therefore, $K=0$.

Let $R$ be a commutative ring and $S \subset R$ be a multiplicative subset such that $\operatorname{pd}_{R} S^{-1} R \leq 1$. Let $0 \longrightarrow A \longrightarrow B \longrightarrow C \longrightarrow 0$ be a short exact sequence of $R$-modules. Applying the cohomological functor $\operatorname{Hom}_{\mathrm{D}(R \text {-mod })}\left(K_{R, S}^{\bullet},-\right)$, we obtain an exact sequence

$$
\begin{aligned}
& 0 \longrightarrow \operatorname{Hom}_{R}\left(S^{-1} R / R, A\right) \longrightarrow \operatorname{Hom}_{R}\left(S^{-1} R / R, B\right) \longrightarrow \\
& \operatorname{Hom}_{R}\left(S^{-1} R / R, C\right) \longrightarrow \Delta_{R, S}(A) \longrightarrow \Delta_{R, S}(B) \longrightarrow \Delta_{R, S}(C) \longrightarrow 0
\end{aligned}
$$

(see Section 4 for the notation).

Notice that, when $R$ is a Noetherian commutative ring, the $S$-torsion in $R$ is bounded for any multiplicative subset $S \subset R$.

Lemma 7.6. Let $R$ be a commutative ring and $S \subset R$ be a multiplicative subset such that the $S$-torsion in $R$ is bounded. Then one has $\operatorname{Hom}_{R}\left(S^{-1} R / R, F\right)=0$ for any flat $R$-module $F$.

Proof. If the $S$-torsion in a commutative $\operatorname{ring} R$ is bounded, then the $S$-torsion in any flat $R$-module $F$ is bounded, too [24, proof of Corollary 2.7]. Furthermore, one has $\operatorname{Hom}_{R}\left(S^{-1} R / R, M\right)=\operatorname{Hom}_{R}\left(S^{-1} R / R, \Gamma_{S}(M)\right)=0$ for any $R$-module $M$ with bounded $S$-torsion, since $\operatorname{Hom}_{R}\left(S^{-1} R / R, N\right) \subset \operatorname{Hom}_{R}\left(S^{-1} R, N\right)=0$ for any $R$-module $N$ annihilated by an element $s \in S$.

Let $R$ be a Noetherian commutative ring and $S \subset R$ be a countable multiplicative subset. The pair of full subcategories ( $R$-flat $S$-contramodule $R$-modules, $R$-cotorsion $S$-contramodule $R$-modules) is called the flat cotorsion theory in the abelian category $R-\bmod _{S \text {-ctra }}$. Having finished the preparatory work, we can now proceed to construct the approximation sequences in the category $R-\bmod _{S \text {-ctra }}$ proving that this is indeed a complete cotorsion theory (as it was promised in Theorem 7.1).

Lemma 7.7. Let $R$ be a Noetherian commutative ring and $S \subset R$ be a countable multiplicative subset. Let $C$ be an $S$-contramodule $R$-module, and let $0 \longrightarrow K \longrightarrow$ $F \longrightarrow C \longrightarrow 0$ be a short exact sequence of $R$-modules with a flat $R$-module $F$. Then there is a short exact sequence of $S$-contramodule $R$-modules

$$
0 \longrightarrow \Delta_{R, S}(K) \longrightarrow \Delta_{R, S}(F) \longrightarrow C \longrightarrow 0
$$

with a flat $R$-module $\Delta_{R, S}(F)$. If $K$ is a cotorsion $R$-module, then the $R$-module $\Delta_{R, S}(K)$ is also cotorsion.

Proof. By Lemma 3.1(b), we have $\Delta_{R, S}(C)=C$. Furthermore, the $R$-module $\operatorname{Hom}_{R}\left(S^{-1} R / R, C\right)$ is a submodule in the $R$-module $\operatorname{Hom}_{R}\left(S^{-1} R, C\right)$, which vanishes by virtue of $C$ being an $S$-contramodule. Hence the desired short exact sequence is a particular case of the exact sequence (5). By Lemmas 7.4 and 7.5, the $R$-module $\Delta_{R, S}(F)$ is flat for any flat $R$-module $F$. 
Finally, we have $\operatorname{Hom}_{R}\left(S^{-1} R / R, F\right)=0$ by Lemma 7.6, and consequently $\operatorname{Hom}_{R}\left(S^{-1} R / R, K\right)=0$. Assume that the $R$-module $K$ is $S$-weakly cotorsion. Then the exact sequence (3) (from Lemma 4.1) for the $R$-module $K$ reduces to a short exact sequence $0 \longrightarrow \operatorname{Hom}_{R}\left(S^{-1} R, K\right) \longrightarrow K \longrightarrow \Delta_{R, S}(K) \longrightarrow 0$.

Now, since the $R$-module $S^{-1} R$ is flat, the $R$-module $\operatorname{Hom}_{R}\left(S^{-1} R, K\right)$ is cotorsion whenever the $R$-module $K$ is [22, Lemma 1.3.2(a)]. Therefore, the $R$-module $\Delta_{R, S}(K)$ is also cotorsion in this case, as the cokernel of an injective morphism of cotorsion $R$-modules.

Lemma 7.8. Let $R$ be a Noetherian commutative ring and $S \subset R$ be a countable multiplicative subset. Let $C$ be an $S$-contramodule $R$-module, and let $0 \longrightarrow C \longrightarrow$ $K \longrightarrow F \longrightarrow 0$ be a short exact sequence of $R$-modules with a flat $R$-module $F$. Then there is a short exact sequence of $S$-contramodule $R$-modules

$$
0 \longrightarrow C \longrightarrow \Delta_{R, S}(K) \longrightarrow \Delta_{R, S}(F) \longrightarrow 0
$$

with a flat $R$-module $\Delta_{R, S}(F)$. If $K$ is a cotorsion $R$-module, then the $R$-module $\Delta_{R, S}(K)$ is also cotorsion.

Proof. For the reasons mentioned in the proof of Lemma 7.7, we have $\Delta_{R, S}(C)=C$ and $\operatorname{Hom}_{R}\left(S^{-1} R / R, F\right)=0$. Hence the desired short exact sequence is a particular case of the exact sequence (5). The $R$-module $\Delta_{R, S}(F)$ is flat for any flat $R$-module $F$, as it was pointed out. Also for the reasons explained in the proof of Lemma 7.7, we have $\operatorname{Hom}_{R}\left(S^{-1} R / R, C\right)=0$. Hence $\operatorname{Hom}_{R}\left(S^{-1} R / R, K\right)=0$, and in the same way as in the proof of Lemma 7.7 one deduces that the $R$-module $\Delta_{R, S}(K)$ is cotorsion whenever the $R$-module $K$ is.

Proof of Theorem 7.1. All the substantial work has been done already in Lemmas 7.7 and 7.8, which produce the required approximation sequences out of the approximation sequences for the flat cotorsion theory on the category of $R$-modules $R$-mod (which exist by [8, Theorem 10] and [6, Proposition 2]).

One can further observe that the functor $\mathrm{Ext}^{1}$ in the abelian category $R$ - $\bmod _{S \text {-ctra }}$ agrees with the functor $\mathrm{Ext}^{1}$ in $R$-mod, since $R$ - $\bmod _{S \text {-ctra }} \subset R$-mod is a full subcategory closed under kernels, cokernels, and extensions. Besides, the full subcategory of $R$-flat objects in $R$ - $\bmod _{S \text {-ctra }}$ is closed under direct summands and kernels of epimorphisms, while the full subcategory of $R$-cotorsion objects in $R$ - $\bmod _{S \text {-ctra }}$ is closed under direct summands and cokernels of monomorphisms, since the full subcategories of flat $R$-modules and cotorsion $R$-modules in the abelian category $R$-mod have similar properties. This observations are sufficient to imply that the pair of full subcategories in $R-\bmod _{S \text {-ctra }}$ which we are interested in is a cotorsion theory/pair, and that this cotorsion theory is complete and hereditary.

Remark 7.9. Notice that, in the context of Lemma 7.7, if the $R$-module $K$ is, at least, $S$-weakly cotorsion, then so is the $R$-module $F$ (because the $R$-module $C$, being an $S$-contramodule, is consequently $S$-weakly cotorsion, and $F$ is an extension of $C$ and $K)$. Hence one has $\Delta_{R, S}(K)=K / h_{S}(K)$ and $\Delta_{R, S}(F)=F / h_{S}(F)$. Similarly, in the context of Lemma 7.8, if the $R$-module $K$ is $S$-weakly cotorsion, then so is the 
$R$-module $F$ (as a quotient $R$-module of $K$ ). Hence one also has $\Delta_{R, S}(K)=K / h_{S}(K)$ and $\Delta_{R, S}(F)=F / h_{S}(F)$. In other words, for a countable multiplicative subset $S$ in a Noetherian commutative ring $R$, in order to produce the approximation sequences for the flat cotorsion theory in $R$ - $\bmod _{S \text {-ctra }}$ from the approximation sequences for the flat cotorsion theory in $R$-mod, all one needs to do is to quotient out the maximal $S$-divisible submodules.

7.2. Quite flat cotorsion theory in the bounded torsion case. Let $R$ be a commutative ring, $S \subset R$ be a multiplicative subset, and $M$ be an $R$-module. The $S$-topology on $M$ is the topology with the base of neighborhoods of zero formed by the submodules $s M \subset M$, where $s \in S$. The $R$-module $\Lambda_{S}(M)=\lim _{s \in S} M / s M$ is the completion of $M$ with respect to the $S$-topology. When $S$ is countable, the topology of projective limit (of discrete $R$-modules $M / s M$ ) on $\Lambda_{S}(M)$ always coincides with the $S$-topology of the $R$-module $\Lambda_{S}(M)$ [24, Proposition 2.2(b) and Theorem 2.3 (i) $\left.\Leftrightarrow(\mathrm{ii})\right]$.

We refer to [21, Section 1.2] or [26, Section 5] for the definition of a left contramodule over a complete, separated topological associative ring $\mathfrak{R}$ with a base of neighborhoods of zero fomed by open right ideals. In the context of this paper, we set $\mathfrak{R}=\Lambda_{S}(R)$, where $R$ is a commutative ring and $S \subset R$ is a countable multiplicative subset. The commutative ring $\mathfrak{R}$ is endowed with its topology of projective limit of discrete commutative rings $\lim _{s \in S} R / s R$ or, which is the same, its $S$-topology (as an $R$-module). We denote the abelian category of $\mathfrak{R}$-contramodules by $\mathfrak{R}$-contra.

The following result is a generalization of [27, Theorem 5.9].

Theorem 7.10. Let $R$ be a commutative ring and $S \subset R$ be a countable multiplicative subset such that the $S$-torsion in $R$ is bounded. Then the forgetful functor $\mathfrak{R}$-contra $\longrightarrow R$-mod induces an equivalence of abelian categories $\mathfrak{R}$-contra $\simeq$ $R-\bmod _{S \text {-ctra }}$.

Proof. In fact, this assertion holds under in the greater generality of a multiplicative subset $S \subset R$ such that $\operatorname{pd}_{R} S^{-1} R \leq 1$ and the $S$-torsion in $R$ is bounded. See [25, Example 2.4(3)]. For a countable multiplicative subset $S \subset R$ such that the $S$-torsion in $R$ is unbounded, the abelian category $\mathfrak{R}$-contra is, generally speaking, a full subcategory in the abelian category $R-\bmod _{S \text {-ctra }}$ with an exact embedding functor $\mathfrak{R}$-contra $\longrightarrow R-\bmod _{S \text {-ctra }}[25$, Example 3.7(2)] (cf. [27, Theorem 5.20]).

It is important for us that, when the multiplicative subset $S \subset R$ is countable, the topological ring $\mathfrak{R}$ has a countable base of neighborhoods of zero, so the results of [26, Sections 6-7] are applicable.

An $\mathfrak{R}$-contramodule $F$ is called flat if the $R / s R$-modules $F / s F$ are flat for all elements $s \in S$ (see [22, Section D.1] or [26, Sections 5-6]). Unlike in the Noetherian case of Section 7.1, there is no claim that flat $\mathfrak{R}$-contramodules are flat $R$-modules in our present generality (when $S$ is just a countable multiplicative subset in a commutative ring $R$ such that the $S$-torsion in $R$ is bounded). 
Lemma 7.11. Let $R$ be a commutative ring and $S \subset R$ be a countable multiplicative subset such that the $S$-torsion in $R$ is bounded. Then, for any flat $R$-module $F$, the $S$-contramodule $R$-module $\Delta_{R, S}(F)$ is a flat $\mathfrak{R}$-contramodule.

Proof. By [24, Lemma 1.11], we have $\Delta_{R, S}(F) / s \Delta_{R, S}(F)=F / s F$, which is a flat $R / s R$-module.

The definitions of an almost cotorsion and a quite flat $R$-module were given in Section 1.6. The following definitions extend these concepts to the realm of $S$-contramodule $R$-modules. For simplicity of notation, let us recall and use the fact that the functor $\mathrm{Ext}^{1}$ in the abelian category $R-\bmod _{S \text {-ctra }}$ agrees with the functor Ext $^{1}$ in the abelian category $R$-mod, as $R-\bmod _{S \text {-ctra }} \subset R$-mod is a full subcategory closed under kernels, cokernels, and extensions.

Let $R$ be a commutative ring and $S \subset R$ be a countable multiplicative subset such that the $S$-torsion in $R$ is bounded. For any countable multiplicative subset $T \subset R$, consider the $S$-contramodule $R$-module $\Delta_{R, S}\left(T^{-1} R\right)$. An $S$-contramodule $R$-module $C$ is said to be almost cotorsion if $\operatorname{Ext}_{R}^{1}\left(\Delta_{R, S}\left(T^{-1} R\right), C\right)=0$ for all countable multiplicative subsets $T \subset R$. An $S$-contramodule $R$-module $F$ is said to be quite flat if $\operatorname{Ext}_{R}^{1}(F, C)=0$ for all almost cotorsion $S$-contramodule $R$-modules $C$.

In other words, these definitions mean that the pair of full subcategories (quite flat $S$-contramodule $R$-modules, almost cotorsion $S$-contramodule $R$-modules) is defined as the cotorsion theory/pair generated by the objects $\Delta_{R, S}\left(T^{-1} R\right)$ in the abelian category $R$ - $\bmod _{S \text {-ctra }}$. This cotorsion theory is called the quite flat cotorsion theory in the abelian category $R$ - $\bmod _{S \text {-ctra }}$.

Once again, there is no claim that quite flat $S$-contramodule $R$-modules are quite flat as $R$-modules (even for a Noetherian ring $R$ ). On the other hand, the following assertions hold.

Proposition 7.12. Let $R$ be a commutative ring and $S \subset R$ be a countable multiplicative subset such that the $S$-torsion in $R$ is bounded. Then all quite flat $S$-contramodule $R$-modules are flat $\mathfrak{R}$-contramodules.

Proof. Both the arguments in [27, proof of Proposition 5.12] are applicable to the situation at hand just as well (proving also in addition that an $S$-contramodule $R$-module is quite flat if and only if it is a direct summand of a transfinitely iterated extension of the objects $\Delta_{R, S}\left(T^{-1} R\right)$ in the category $R$ - $\bmod _{S \text {-ctra }}$, in the sense of the inductive limit, or more precisely, of [26, Definition 4.3]).

Theorem 7.13. Let $R$ be a commutative ring and $S \subset R$ be a countable multiplicative subset such that the $S$-torsion in $R$ is bounded. Then an $S$-contramodule $R$-module is almost cotorsion if and only if it is almost cotorsion as an $R$-module.

Proof. The proof of this theorem does not really use the condition of countability of the multiplicative subset $S \subset R$, but only the conditions that the $S$-torsion in $R$ is bounded and that the projective dimension of the $R$-module $S^{-1} R$ does not exceed 1 . We will construct an isomorphism of the Ext modules

$$
\operatorname{Ext}_{R}^{1}(F, C) \simeq \operatorname{Ext}_{R-\bmod _{S-\mathrm{ctra}}}^{1}\left(\Delta_{R, S}(F), C\right)
$$


for any flat $R$-module $F$ and any $S$-contramodule $R$-module $C$. Specializing to the case of the flat $R$-module $F=T^{-1} R$ will then immediately imply the assertion of the theorem.

Suppose that we have short exact sequence of $R$-modules

$$
0 \longrightarrow \mathrm{C} \longrightarrow \mathrm{B} \longrightarrow \mathrm{F} \longrightarrow 0,
$$

where $F$ is a flat $R$-module and $C$ is an $S$-contramodule $R$-module. Applying the functor $\Delta_{R, S}$ and recalling the exact sequence (5) from Section 7.1 together with Lemma 7.6, we get a short exact sequence of $S$-contramodule $R$-modules

$$
0 \longrightarrow C \longrightarrow \Delta_{R, S}(B) \longrightarrow \Delta_{R, S}(F) \longrightarrow 0,
$$

because $\Delta_{R, S}(C)=C$ by Lemma 3.1(b). There is a natural adjunction morphism from the short exact sequence (6) to the short exact sequence (7), hence it follows that the sequence (6) is the pullback of the sequence (7) with respect to the adjunction morphism $F \longrightarrow \Delta_{R, S}(F)$.

Conversely, given a short exact sequence of $S$-contramodule $R$-modules

$$
0 \longrightarrow C \longrightarrow B^{\prime} \longrightarrow \Delta_{R, S}(F) \longrightarrow 0,
$$

one can take the pullback with respect to the morphism $F \longrightarrow \Delta_{R, S}(F)$ in order to produce a short exact sequence of $R$-modules (6). Then there is a natural morphism of short exact sequences of $R$-modules from the exact sequence (6) into the exact sequence (8); and the adjunction morphisms provide an isomorphism from the exact sequence $(7)$ to the exact sequence (8).

Theorem 7.14. Let $R$ be a commutative ring and $S \subset R$ be a countable multiplicative subset such that the $S$-torsion in $R$ is bounded. Then the pair of full subcategories (quite flat $S$-contramodule $R$-modules, almost cotorsion $S$-contramodule $R$-modules) is a hereditary complete cotorsion theory in the abelian category $R-\bmod _{S \text {-ctra }}$.

Proof. Since the $R$-module $T^{-1} R$ is flat for any (countable) multiplicative subset $T \subset R$, the $\mathfrak{R}$-contramodule $\Delta_{R, S}\left(T^{-1} R\right)$ is flat by Lemma 7.11. Identifying the category $R-\bmod _{S \text {-ctra }}$ with the category $\mathfrak{R}$-contra by Theorem 7.10 , we can apply the result of [26, Corollary 7.11], according to which any cotorsion theory generated by a set of flat $\mathfrak{R}$-contramodules is complete in $\mathfrak{R}$-contra.

To show that the quite flat cotorsion theory in $R$ - $\bmod _{S \text {-ctra }}$ is hereditary, we will check that the objects $\Delta_{R, S}\left(T^{-1} R\right)$ have projective dimension at most 1 in $R-\bmod _{S \text {-ctra }}$. This will prove the stronger claim that the class of almost cotorsion $S$-contramodule $R$-modules is closed under the passages to arbitrary quotient objects in $R$ - $\bmod _{S \text {-ctra }}$, while all quite flat $S$-contramodule $R$-modules have projective dimension at most 1 as objects of $R-\bmod _{S \text {-ctra }}$.

Indeed, let $0 \longrightarrow Q \longrightarrow P \longrightarrow T^{-1} R \longrightarrow 0$ be a projective resolution of the $R$-module $T^{-1} R$. Applying the functor $\Delta_{R, S}$ and using the exact sequence (5) together with Lemma 7.6, we obtain a short exact sequence of $S$-contramodule $R$-modules $0 \longrightarrow \Delta_{R, S}(Q) \longrightarrow \Delta_{R, S}(P) \longrightarrow \Delta_{R, S}\left(T^{-1} R\right) \longrightarrow 0$. The functor 
$\Delta_{R, S}: R-\bmod \longrightarrow R-\bmod _{S \text {-ctra }}$ is left adjoint to an exact functor, so it takes projectives to projectives. Thus we have obtained the desired two-term projective resolution of our object $\Delta_{R, S}\left(T^{-1} R\right) \in R$ - $\bmod _{S \text {-ctra }}$.

7.3. Separated $S$-contramodule $R$-modules. Let $R$ be a commutative ring and $S \subset R$ be a multiplicative subset. An $R$-module $C$ is said to be $S$-complete if the natural map

$$
\lambda_{R, S, C}: C \longrightarrow \Lambda_{R, S}(C)=\lim _{s \in S} C / s C
$$

is surjective, and $S$-separated if the map $\lambda_{R, S, C}$ is injective.

Clearly, an $R$-module $C$ is $S$-separated if and only if the intersection $\bigcap_{s \in S} s C \subset C$ vanishes. It follows that any $R$-submodule of an $S$-separated $R$-module is $S$-separated.

For any multiplicative subset $S$ in a commutative ring $R$ such that $\operatorname{pd}_{R} S^{-1} R \leq 1$, any $S$-separated and $S$-complete $R$-module is an $S$-contramodule [24, Lemma 2.1(a)]. It follows from our Lemmas 3.1(b) and 3.2 that, for any countable multiplicative subset $S \subset R$, any $S$-contramodule $R$-module is $S$-complete.

The following corollaries pick out the aspects of the results of Sections 7.1-7.2 relevant for the proofs of Main Lemmas 1.22 and 1.24 in Section 8. We recall that, according to our terminology introduced in the beginning of Section 7.1, an $S$-contramodule $R$-module is called $R$-almost cotorsion if it is almost cotorsion as an $R$-module.

Corollary 7.15. Let $R$ be a Noetherian commutative ring, $S \subset R$ be a countable multiplicative subset, and $C$ be an $R$-almost cotorsion $S$-contramodule $R$-module. Then the $R$-module $C$ can be presented as the cokernel of an injective morphism of $R$-almost cotorsion $S$-separated $S$-complete $R$-modules.

Proof. By Theorem 7.1, or more specifically by Lemma 7.7, there exists a short exact sequence of $S$-contramodule $R$-modules $0 \longrightarrow K \longrightarrow F \longrightarrow C \longrightarrow 0$, where the $R$-mdoule $F$ is flat and the $R$-module $K$ is cotorsion. What is important for us is that the $R$-module $K$ is almost cotorsion; since the $R$-module $C$ is almost cotorsion by assumption, it follows that the $R$-module $F$ is almost cotorsion, too.

Furthermore, any $R$-flat $S$-contramodule $R$-module is $S$-separated by Lemma 7.5. The $R$-module $F$ being $S$-separated, it follows that its submodule $K$ is $S$-separated, too. Thus $K \longrightarrow F$ is an injective morphism of $R$-almost cotorsion $S$-separated $S$-contramodules with the cokernel $C$.

Corollary 7.16. Let $R$ be a commutative ring and $S \subset R$ be a countable multiplicative subset such that the $S$-torsion in $R$ is bounded. Then any $R$-almost cotorsion $S$-contramodule $R$-module can be presented as the cokernel of an injective morphism of $R$-almost cotorsion $S$-separated $S$-complete $R$-modules.

Proof. By Theorem 7.14, the quite flat cotorsion theory in $R$ - $\bmod _{S \text {-ctra }}$ is complete. Hence for any $S$-contramodule $R$-module $C$ there exists a short exact sequence $0 \longrightarrow$ $K \longrightarrow F \longrightarrow C \longrightarrow 0$, where $K$ is an almost cotorsion $S$-contramodule $R$-module and $F$ is a quite flat $S$-contramodule $R$-module. 
By Theorem 7.13, $K$ is an almost cotorsion $R$-module. Assuming that $C$ is an almost cotorsion $R$-module, we can conclude that the $R$-module $F$ is almost cotorsion, too. By Proposition 7.12, $F$ is a flat $\mathfrak{R}$-contramodule. By [22, Corollary D.1.7] or [26, Corollary 6.15], all flat $\mathfrak{R}$-contramodules are $S$-separated. So the $R$-module $F$ is $S$-separated, and it follows that its submodule $K$ is $S$-separated, too.

Thus $K \longrightarrow F$ is an injective morphism of $R$-almost cotorsion $S$-separated $S$-contramodule $R$-modules with the cokernel $C$.

Remark 7.17. Just as in [27, Sections 5.5-5.6], for any countable multiplicative subset $S$ in a commutative ring $R$ one can define the full subcategory of quotseparated $S$-contramodule $R$-modules $R$ - $\bmod _{S \text {-ctra }}^{\text {qs }} \subset R$-mod and show that it is equivalent to the abelian category $\mathfrak{R}$-contra. The one can proceed to construct, following essentially the exposition in [22, Section D.4] with the words "very flat" replaced by "quite flat" and the word "contraadjusted" replaced by "almost cotorsion", and with the same simplifications as in [27], the quite flat cotorsion theory on the abelian category $R$ - $\bmod _{S \text {-ctra }}^{\text {qs }}$. In the same way as in [27], it follows that any $R$-almost cotorsion quotseparated $S$-contramodule $R$-module is the cokernel of an injective morphism of $R$-almost cotorsion $S$-separated $S$-complete $R$-modules. We omit the details.

\section{Quite Flat and Almost Cotorsion Modules}

Let us start from recalling the definitions given in Section 1.6. Let $R$ be a commutative ring. We say that an $R$-module $C$ is almost cotorsion if $\operatorname{Ext}_{R}^{1}\left(S^{-1} R, C\right)=0$ for all at most countable multiplicative subsets $S \subset R$. An $R$-module $F$ is said to be quite flat if $\operatorname{Ext}_{R}^{1}(F, C)=0$ for all almost cotorsion $R$-modules $C$.

An $R$-module $F$ is quite flat if and only if it is a direct summand of a transfinitely iterated extension, in the sense of the inductive limit, of $R$-modules isomorphic to $S^{-1} R$, where $S \subset R$ are (at most) countable multiplicative subsets [13, Corollary 6.14]. The pair of full subcategories (quite flat $R$-modules, almost cotorsion $R$-modules) is called the quite flat cotorsion theory in $R$-mod.

The following four lemmas list the general properties of the classes of almost cotorsion and quite flat modules over commutative rings.

Lemma 8.1. (a) For any commutative ring $R$, the class of all almost cotorsion $R$-modules is closed under extensions, quotients (by arbitrary submodules), infinite products, and transfinitely iterated extensions in the sense of the projective limit in the category $R$-mod.

(b) For any commutative ring $R$, the class of all quite flat $R$-modules is closed under extensions, kernels of surjective morphisms, direct summands, infinite direct sums, and transfinitely iterated extensions in the sense of the inductive limit in the category $R$-mod.

(c) The projective dimension of any quite flat $R$-module does not exceed 1 . 
Proof. The properties of closedness with respect to quotients in part (a) and kernels of surjective morphisms in part (b) follow from the fact that $\operatorname{pd}_{R} S^{-1} R \leq 1$ for all countable multiplicative subsets $S \subset R$. So does the assertion of part (c). All the other assertions are general properties of Ext ${ }^{1}$-orthogonal classes in $R$-mod $[8$, Lemma 1 and Proposition 18].

Lemma 8.2. (a) For any commutative ring $R$, the class of quite flat $R$-modules is closed with respect to the tensor products over $R$.

(b) For any commutative ring $R$, any quite flat $R$-module $F$, and any almost cotorsion $R$-module $C$, the $R$-module $\operatorname{Hom}_{R}(F, C)$ is almost cotorsion.

Proof. Similar to the proof of [22, Lemma 1.2.1], using the isomorphism $\operatorname{Ext}_{R}^{1}\left(F \otimes_{R}\right.$ $G, C) \simeq \operatorname{Ext}_{R}^{1}\left(G, \operatorname{Hom}_{R}(F, C)\right)$, holding for any $R$-module $G$, any quite flat $R$-module $F$, and any almost cotorsion $R$-module $C$.

Lemma 8.3. Let $f: R \longrightarrow R^{\prime}$ be a homomorphism of commutative rings. Then

(a) any almost cotorsion $R^{\prime}$-module is also an almost cotorsion $R$-module in the $R$-module structure obtained by the restriction of scalars via $f$;

(b) if $F$ is a quite flat $R$-module, then the $R^{\prime}$-module $R^{\prime} \otimes_{R} F$ obtained by the extension of scalars via $f$ is also quite flat.

Proof. Both the assertions hold, essentially, because for any (at most) countable multiplicative subset $S \subset R$, the image $S^{\prime}=f(S) \subset R^{\prime}$ of the multiplicative subset $S$ under the map $f$ is an (at most) countable multiplicative subset $S^{\prime} \subset R^{\prime}$, and the $R^{\prime}$-algebra/module $R^{\prime} \otimes_{R} S^{-1} R$ is isomorphic to $S^{\prime-1} R^{\prime}$ (cf. [22, Lemma 1.2.2(a-b)]).

Lemma 8.4. Let $f: R \longrightarrow R^{\prime}$ be a homomorphism of commutative rings such that for every element $r^{\prime} \in R^{\prime}$ there exist an element $r \in R$ and an invertible element $u \in R^{\prime}$ for which $r^{\prime}=u f(r)$. Then an $R^{\prime}$-module is almost cotorsion if and only if it is almost cotorsion as an $R$-module.

Proof. The "only if" assertion holds by Lemma 8.3. To prove the "if", suppose that we are given a countable multiplicative subset $S^{\prime} \subset R^{\prime}$. For every element $s^{\prime} \in S^{\prime}$, choose an element $s \in R$ such that there exists an invertible element $u \in R^{\prime}$ for which $s^{\prime}=u f(s)$. Let $S \subset R$ be the multiplicative subset generated by all the elements $s \in R$ so obtained. Then $S$ is a countable multiplicative subset in $R$ and we have $R^{\prime} \otimes_{R} S^{-1} R=S^{\prime-1} R^{\prime}$. Now if $C$ is an $R^{\prime}$-module that is almost cotorsion as an $R$-module, then $\operatorname{Ext}_{R^{\prime}}^{1}\left(S^{\prime-1} R^{\prime}, C\right)=\operatorname{Ext}_{R^{\prime}}^{1}\left(R^{\prime} \otimes_{R} S^{-1} R, C\right)=\operatorname{Ext}_{R}^{1}\left(S^{-1} R, C\right)=0$, hence $C$ is an almost cotorsion $R^{\prime}$-module.

In view of Lemma 8.1(c), it follows from Theorem 1.17 that the projective dimension of a flat module over a commutative Noetherian ring with countable spectrum cannot exceed 1. Let us present a simpler alternative proof of this result before proceeding to prove Theorem 1.17 .

Lemma 8.5. Let $S$ and $T \subset R$ be two multiplicative subsets in a commutative ring $R$. Assume that, for any prime ideal $\mathfrak{p} \subset R$, one has $\mathfrak{p} \cap S \neq \varnothing$ if and only if $\mathfrak{p} \cap T \neq \varnothing$. Then the two $R$-algebras $S^{-1} R$ and $T^{-1} R$ are naturally isomorphic. 
Proof. It suffices to check that an element $r \in R$ is invertible in $S^{-1} R$ if and only if it is invertible in $T^{-1} R$. Indeed, if an element $r \in R$ is not invertible in $S^{-1} R$, then there exists a maximal ideal $\mathfrak{m} \subset S^{-1} R$ such that the image of $r$ in $S^{-1} R$ belongs to $\mathfrak{m}$. Denote by $\mathfrak{p} \subset R$ the full preimage of the maximal ideal $\mathfrak{m} \subset S^{-1} R$ under the localization morphism $R \longrightarrow S^{-1} R$. Then $\mathfrak{p}$ is a prime ideal in $R$ such that $r \in \mathfrak{p}$ and $\mathfrak{p} \cap S=\varnothing$. Conversely, if $\mathfrak{p} \subset R$ is a prime ideal such that $\mathfrak{p} \cap S=\varnothing$, then the extension of $\mathfrak{p}$ in $S^{-1} R$ is not the unit ideal in $S^{-1} R$; so if $r \in \mathfrak{p}$ then $r$ is not invertible in $S^{-1} R$. Thus an element $r \in R$ becomes invertible in $S^{-1} R$ if and only if one has $\mathfrak{p} \cap S \neq \varnothing$ for all prime ideals $\mathfrak{p} \subset R$ containing $r$. As the set of all elements $r \in R$ that become invertible in $T^{-1} R$ can be described similarly, the assertion follows.

We refer to the dissertation [7, Section 3.2] for a general discussion of Noetherian rings with small spectrum.

Lemma 8.6. Let $R$ be a commutative ring with (at most) countable spectrum, and let $S \subset R$ be a multiplicative subset. Then there exists an (at most) countable multiplicative subset $T \subset S$ such that $T^{-1} R=S^{-1} R$.

Proof. It suffices to choose, for every prime ideal $\mathfrak{p} \subset R$ such that $\mathfrak{p} \cap S \neq \varnothing$, an element $t \in \mathfrak{p} \cap S$. Set $T \subset R$ to be multiplicative subset generated by all the elements $t \in R$ so obtained. Then the multiplicative subset $T$ is (at most) countable, it is contained in $S$, and $T^{-1} R=S^{-1} R$ by Lemma 8.5.

Theorem 8.7. For any Noetherian commutative ring $R$, the supremum of projective dimensions of flat $R$-modules is equal to the supremum of projective dimensions of the $R$-modules $S^{-1} R$ over all multiplicative subsets $S \subset R$.

Proof. This is [28, Théorème II.3.3.1] (see [15, §1] for a correction of a mistake in the exposition in [28]).

Corollary 8.8. Let $R$ be a Noetherian commutative ring with countable spectrum. Then the projective dimension of any flat $R$-module does not exceed 1 .

Proof. According to Lemma 8.6 and [24, Lemma 1.9], the projective dimension of the $R$-module $S^{-1} R$ does not exceed 1 for any multiplicative subset $S \subset R$. Thus it remains to apply Theorem 8.7.

Alternatively, one can use Theorem 1.17 and Lemma 8.1(c).

First proof of Theorem 1.17. Let us show that Theorem 1.17 follows from Main Lemma 1.18. Clearly, for any element $r \in R$, the quotient ring $R / r R$ is a Noetherian ring with (at most) countable spectrum. Proceeding by Noetherian induction (cf. the proof of [27, Main Theorem 1.3] in [27, Section 2]), we can assume that, for any nonzero element $r \in R$, all flat $R / r R$-modules are quite flat.

Let $\mathfrak{q}_{1}, \ldots, \mathfrak{q}_{k} \subset R$ be the minimal prime ideals in $R$. Set $S=R \backslash \bigcup_{i=1}^{k} \mathfrak{q}_{k} \subset R$. Then $S$ is a multiplicative subset in $R$ and the ring $S^{-1} R$ is Artinian, so all flat $S^{-1} R$-modules are projective. By Lemma 8.6, there exists a countable multiplicative subset $T \subset S$ such that $T^{-1} R=S^{-1} R$. 
Let $F$ be a flat $R$-module. Then the the $R / t R$-module $F / t F$ is flat for all $t \in T$ and the $T^{-1} R$-module $T^{-1} F$ is flat. By the Noetherian induction assumption, it follows that the $R / t R$-module $F / t F$ is quite flat; and all flat $T^{-1} R$-modules are projective, hence also quite flat. Applying Main Lemma 1.18 to the Noetherian commutative ring $R$ with the countable multiplicative subset $T \subset R$ and the flat $R$-module $F$, we conclude that the $R$-module $F$ is quite flat.

Second proof of Theorem 1.17. Theorem 1.17 follows immediately from (is, in fact, an equivalent restatement of) Corollary 1.20.

Proof of Corollaries 1.20 and 1.21. It was essentially already explained in Section 1.6 that Proposition 1.19 implies Corollaries 1.20 and 1.21, but let us repeat this explanation here adding a couple of references. The point is that all the $R$-modules right 1-obtainable from vector spaces over the residue fields of prime ideals in $R$ are not only almost cotorsion but, in fact, cotorsion. Indeed, all vector spaces are obviously cotorsion as modules over their respective fields, all restrictions of scalars take cotorsion modules to cotorsion modules [22, Lemma 1.3.4(a)], and all $R$-modules right 1-obtainable from cotorsion $R$-modules are cotorsion (by Lemma 2.4).

Proof of Proposition 1.19. The "if" part has been already explained in the preceding proof. One deduces the "only if" from Main Lemma 1.22 proceeding by Noetherian induction on the ring $R$.

Assume that, for all nonzero elements $r \in R$, all almost cotorsion $R / r R$-modules are right 1-obtainable from vector spaces over the residue fields of prime ideals of $R / r R$. As in the first proof of Theorem 1.17 above, one can find a countable multiplicative subset $T \subset R$ such that $0 \notin T$ and the ring of fractions $T^{-1} R$ is Artinian. Then all $T^{-1} R$-modules are obtainable as finitely iterated extensions of modules over the residue fields of the maximal ideals of $T^{-1} R$.

By Main Lemma 1.22, all almost cotorsion $R$-modules are right 1-obtainable from almost cotorsion $R / t R$-modules, $t \in T$, and almost cotorsion $T^{-1} R$-modules. It remains to recall that all the residue fields of the rings $R / t R$ and $T^{-1} R$ are, at the same time, residue fields of the ring $R$.

Proof of Main Lemmas 1.18 and 1.23. The "only if" assertions hold for any multiplicative subset $S$ in a commutative ring $R$ by Lemma 8.3(b). The "if" assertions of Main Lemmas 1.18 and 1.23 are deduced from the "only if" assertions of Main Lemmas 1.22 and 1.24 , respectively.

Under any of the respective assumptions on $R$ and/or $S$, consider a flat $R$-module $F$ such that the $R / s R$-module $F / s F$ is quite flat for all $s \in S$ and the $S^{-1} R$-module $S^{-1} F$ is quite flat. In order to show that the $R$-module $F$ is quite flat, we will check that $\operatorname{Ext}_{R}^{1}(F, C)=0$ for all almost cotorsion $R$-modules $C$.

Denote by $\mathrm{E}$ the class of all almost cotorsion $R / s R$-modules, $s \in S$, and all almost cotorsion $S^{-1} R$-modules (viewed as $R$-modules via the restriction of scalars). By Lemmas 4.3 and 8.1(c), we have $\operatorname{Ext}_{R}^{i}(F, E)=0$ for all $R$-modules $E \in \mathrm{E}$ and all $i>0$. According to Main Lemma 1.22 or 1.24 , all almost cotorsion $R$-modules 
are right 1-obtainable from $\mathrm{E}$. By virtue of Lemma 2.4, it follows it follows that $\operatorname{Ext}_{R}^{i}(F, C)=0$ for all almost cotorsion $R$-modules $C$.

Main Lemmas 1.22 and 1.24 are deduced from Corollaries 7.15 and 7.16 from Section 7.3. Before proceeding with this proof, we will need one more lemma.

Lemma 8.9. Let $R$ be a commutative ring and $S \subset R$ be a countable multiplicative subset. Then any $R$-almost cotorsion $S$-separated $S$-complete $R$-module is an infinitely iterated extension, in the sense of projective limit, of almost cotorsion $R / s R$-modules, where $s \in S$.

Proof. We will use the notation from Section 3.1. Let $C$ be an $R$-almost cotorsion $S$-separated $S$-complete $R$-module. Then we have $C=\lim _{s \in S} C / s C=\lim _{n \geq 1} C / t_{n} C$. Furthermore, we have exact sequences

$$
C / s_{n} C \stackrel{t_{n-1}}{\longrightarrow} C / t_{n} C \longrightarrow C / t_{n-1} C \longrightarrow 0,
$$

showing that the kernels of the projection maps $C / t_{n} C \longrightarrow C / t_{n-1} C$ are quotient modules of the $R$-modules $C / s C, s \in S$. Finally, it remains to recall that all quotient modules of an almost cotorsion $R$-module $C$ are almost cotorsion $R$-modules by Lemma $8.1(\mathrm{a})$, and all $R / s R$-modules that are almost cotorsion $R$-modules are also almost cotorsion $R / s R$-modules by Lemma 8.4.

Proof of Main Lemmas 1.22 and 1.24. The "if" assertions hold for any multiplicative subset $S$ in a commutative ring $R$. Indeed, all almost cotorsion $R / s R$-modules and almost cotorsion $S^{-1} R$-modules are almost cotorsion $R$-modules by Lemma 8.3(a), and all $R$-modules right 1-obtainable from almost cotorsion $R$-modules are almost cotorsion by Lemmas 2.4 and 8.1(c). The nontrivial part is the "only if".

Denote by $\mathrm{E} \subset R$-mod the class of all almost cotorsion $R / s R$-modules, $s \in S$, and all almost cotorsion $S^{-1} R$-modules (viewed as $R$-modules via the restriction of scalars). Let $C$ be an almost cotorsion $R$-module; we need to show that $C$ is right 1-obtainable from E. Our argument is based on the exact sequence (3) from Section 4 (which is applicable because an almost cotorsion $R$-module $C$ is $S$-weakly cotorsion for a countable multiplicative subset $S \subset R$, by the definition).

In order to prove that $C$ is right 1-obtainable from $\mathrm{E}$, it suffices to check that the $R$-modules $\operatorname{Hom}_{R}\left(S^{-1} R, C\right)$ and $\Delta_{R, S}(C)$ are right 1-obtainable from $\mathrm{E}$, while the $R$-module $\operatorname{Hom}_{R}\left(S^{-1} R / R, C\right)$ is right 2-obtainable from E. Let us consider these three $R$-modules one by one.

The $R$-module $\operatorname{Hom}_{R}\left(S^{-1} R, C\right)$ is almost cotorsion by Lemma 5.6, hence it is an almost cotorsion $S^{-1} R$-module by Lemma 8.4. So the $R$-module $\operatorname{Hom}_{R}\left(S^{-1} R, C\right)$ already belongs to $\mathrm{E}$.

The $R$-module $\Delta_{R, S}(C)$ is almost cotorsion as a quotient module of an almost cotorsion $R$-module $C$ (by Lemma 8.1(a)). Besides, it is an $S$-contramodule $R$-module (by Lemma 3.1(b)). Applying Corollary 7.15 or 7.16 , we can present $\Delta_{R, S}(C)$ as the cokernel of an injective morphism of $R$-almost cotorsion $S$-separated $S$-complete $R$-modules $K \longrightarrow L$. By Lemma 8.9, both $K$ and $L$ are obtainable as infinitely 
iterated extensions of almost cotorsion $R / s R$-modules, $s \in S$. Thus the $R$-module $\Delta_{R, S}(C)$ is simply right obtainable from $\mathrm{E}$.

The $R$-module $\operatorname{Hom}_{R}\left(S^{-1} R / R, C\right)$ is an $S$-contramodule by Lemma 4.2 (and in fact even an $S$-separated $S$-complete $R$-module, as a projective limit of $S$-separated $S$-complete $R$-modules). So it is simply right obtainable from $R / s R$-modules, $s \in$ $S$, by Lemma 3.4 (Lemma 3.3(a) is sufficient). By Lemma 2.6, all $R / s R$-modules are right 2-obtainable from almost cotorsion $R / s R$-modules. Hence the $R$-module $\operatorname{Hom}_{R}\left(S^{-1} R / R, C\right)$ is right 2-obtainable from $\mathrm{E}$.

Remark 8.10. Notice that, for a Noetherian commutative ring of finite Krull dimension with countable spectrum, Theorem 1.16 provides a result stronger than Theorem 1.17. Indeed, Theorem 1.17 uses the localizations with respect to all countable multiplicative subsets $S \subset R$ in order to generate the flat cotorsion theory in $R$-mod, while Theorem 1.16 offers a finite collection of such multiplicative subsets. Nevertheless, these are two different theorems with completely different proofs (one of them presented in Section 6 and the other one in this Section 8). Moreover, we do not know how to extend our proof of Theorem 1.16 to the case of Noetherian rings of infinite Krull dimension, as it is based on Theorem 1.10, which only works for finite collections of multiplicative subsets.

Still, how many multiplicative subsets are actually needed in Theorem 1.17? The answer is: a countable number. For any Noetherian commutative ring $R$ with countable spectrum, there exists a countable collection of countable multiplicative subsets $S_{1}, S_{2}, S_{3}, \ldots \subset R$ such that all $R$-modules $C$ for which $\operatorname{Ext}_{R}^{1}\left(S_{j}^{-1} R, C\right)=0$ for all $j=1,2,3, \ldots$ are cotorsion.

To prove this assertion, one only needs to follow the Noetherian induction argument in the first proof of Theorem 1.17 or in the proof of Proposition 1.19 above. Starting from a Noetherian commutative ring $R$ with countable spectrum, it produces a countable multiplicative subset $T \subset R$ such that the $\operatorname{ring} T^{-1} R$ is Artinian. Then it passes from the ring $R$ to the countable collection of rings $R / t R, t \in T$, and repeats the procedure with each of them. What one obtains in this way is a rooted tree in which the degree of any vertex is at most countable and (due to Noetherianity) there is no infinite branch. By a countable version of the König Lemma, such a tree is countable. In each of its vertices, there sits a quotient ring of the original ring $R$ by a certain ideal and a countable multiplicative subset in this quotient ring. All one needs to do is to lift these multiplicative subsets in the quotient rings to countable multiplicative subsets in $R$, using the procedure from the proof of Lemma 8.4. This produces the desired collection of multiplicative subsets $S_{j} \subset R$.

One needs to follow the argument all the way down to the proof of Main Lemma 1.22 in order to convince oneself that all the $R$-modules that are $S_{j}$-weakly cotorsion for all $j \geq 1$ are right 1-obtainable from vector spaces over the residue fields of the spectrum points of $R$. Then it follows that all such $R$-modules are cotorsion. 


\section{REFERENCES}

[1] L. Angeleri Hügel, D. Herbera, J. Trlifaj. Divisible modules and localizations. J. Algebra 294, \#2, p. 519-551, 2005.

[2] H. Bass. Finitistic dimension and a homological generalization of semi-primary rings. Trans. Amer. Math. Soc. 95, p. 466-488, 1960.

[3] S. Bazzoni, L. Positselski. $S$-almost perfect commutative rings. Electronic preprint 1801.04820 [math. AC].

[4] S. Bazzoni, L. Salce. Strongly flat covers. J. London Math. Soc. 66, \#2, p. 276-294, 2002.

[5] S. Bazzoni, L. Salce. On strongly flat modules over integral domains. Rocky Mountain J. Math. 34, \#2, p. 417-439, 2004.

[6] L. Bican, R. El Bashir, E. Enochs. All modules have flat covers. Bull. London Math. Soc. 33, \#4, p. 385-390, 2001.

[7] C. H. Colbert. Cardinality restrictions on Noetherian spectra. Ph. D. Dissertation, University of Texas at Austin, May 2017.

[8] P. C. Eklof, J. Trlifaj. How to make Ext vanish. Bull. London Math. Soc. 33, \#1, p. 41-51, 2001.

[9] E. Enochs. Flat covers and flat cotorsion modules. Proc. Amer. Math. Soc. 92, \#2, p. 179-184, 1984.

[10] L. Fuchs, L. Salce. S-divisible modules over domains. Forum Math. 4, \#4, p. 383-394, 1992.

[11] L. Fuchs, L. Salce. Modules over non-Noetherian domains. Mathematical Surveys and Monographs 84, American Math. Society, 2001.

[12] L. Fuchs, L. Salce. Almost perfect commutative rings. J. Pure Appl. Algebra 222, \#12, p. 42234238, 2018.

[13] R. Göbel, J. Trlifaj. Approximations and endomorphism algebras of modules. Second Revised and Extended Edition. De Gruyter Expositions in Mathematics 41, De Gruyter, Berlin-Boston, 2012.

[14] V. E. Govorov. On flat modules (Russian). Sibir. Mat. Zh. 6, p. 300-304, 1965.

[15] L. Gruson. Dimension homologique des modules plats sur un anneau commutatif noetherien. Convegno di Algebra Commutativa, INDAM, Rome, 1971, Symposia Mathematica XI, Academic Press, London, 1973, p. 243-254.

[16] R. M. Hamsher. On the structure of a one-dimensional quotient field. J. Algebra 19, \#3, p. 416-425, 1971.

[17] D. K. Harrison. Infinite abelian groups and homological methods. Ann. of Math. 69, \#2, p. 366-391, 1959. Correction, Ann. of Math. 71, \#1, p. 197, 1960.

[18] D. Lazard. Autour de la platitude. Bull. Soc. Math. France 97, p. 81-128, 1969.

[19] S. McAdam. Intersections of height 2 primes. J. Algebra 49, \#2, p. 315-321, 1977.

[20] E. Matlis. Cotorsion modules. Mem. Amer. Math. Soc. 49, 1964.

[21] L. Positselski. Weakly curved $\mathrm{A}_{\infty}$-algebras over a topological local ring. Electronic preprint arXiv:1202.2697 [math.CT].

[22] L. Positselski. Contraherent cosheaves. Electronic preprint arXiv:1209.2995 [math.CT].

[23] L. Positselski. Contraadjusted modules, contramodules, and reduced cotorsion modules. Moscow Math. J. 17, \#3, p. 385-455, 2017. arXiv:1605.03934 [math.CT]

[24] L. Positselski. Triangulated Matlis equivalence. J. Algebra Appl. 17, \#4, article ID 1850067, 2018. arXiv:1605.08018 [math.CT]

[25] L. Positselski. Abelian right perpendicular subcategories in module categories. Electronic preprint arXiv: 1705.04960 [math.CT] .

[26] L. Positselski, J. Rosický. Covers, envelopes, and cotorsion theories in locally presentable abelian categories and contramodule categories. J. Algebra 483, p. 83-128, 2017. arXiv:1512.08119 [math.CT] 
[27] L. Positselski, A. Slávik. Flat morphisms of finite presentation are very flat. Electronic preprint arXiv:1708.00846 [math.AC].

[28] M. Raynaud, L. Gruson. Critères de platitude et de projectivité: Techniques de "platification" d'un module. Invent. Math. 13, \#1-2, p. 1-89, 1971.

[29] L. Salce. Cotorsion theories for abelian groups. Conference on abelian groups and their relationship to the theory of modules, INDAM, Rome, 1977, Symposia Mathematica XXIII, Academic Press, London, 1979, p. 11-32.

[30] J. Šaroch, J. Št'ovíček. The countable telescope conjecture for module categories. Adv. Math. 219, \#3, p. 1002-1036, 2008. arXiv:0801.3936 [math.RA]

[31] J. Trlifaj. Cotorsion theories induced by tilting and cotilting modules. In: Abelian groups, rings and modules (Perth, 2000), Contemp. Math. 273, AMS, Providence, 2001, p. 285-300.

[32] J. Trlifaj. Covers, envelopes, and cotorsion theories. Lecture notes for the workshop "Homological methods in module theory", Cortona, September 2000, 39 pp. Available from http://matematika.cuni.cz/dl/trlifaj/NALG077cortona.pdf

[33] A. Yekutieli. Flatness and completion revisited. Algebr. Represent. Theory 21, \#4, p. 717-736, 2018. arXiv:1606.01832 [math.AC]

Department of Mathematics, Faculty of Natural Sciences, University of Haifa, Mount Carmel, Haifa 31905, Israel; and

Laboratory of Algebraic Geometry, National Research University Higher School of ECONOMics, Moscow 119048; AND

Sector of Algebra and Number Theory, Institute for Information Transmission Problems, Moscow 127051, Russia; and

Charles University, Faculty of Mathematics and Physics, Department of Algebra, Sokolovská 83, 18675 Prague 8, Czech Republic

E-mail address: positselski@yandex.ru

A.S.: Charles University, Faculty of Mathematics and Physics, Department of Algebra, Sokolovská 83, 18675 Prague 8, Czech Republic

E-mail address: Slavik.Alexander@seznam.cz 